Algebraic 83 Geometric $\mathcal{T}$ opology

Volume 5 (2005) 1223-1290

Published: 5 October 2005

ATG

\title{
Hopf algebra structure on topological Hochschild homology
}

\author{
VigLeik AngeltVeit \\ JOHN ROGNES
}

\begin{abstract}
The topological Hochschild homology $T H H(R)$ of a commutative $S$-algebra $\left(E_{\infty}\right.$ ring spectrum) $R$ naturally has the structure of a commutative $R$-algebra in the strict sense, and of a Hopf algebra over $R$ in the homotopy category. We show, under a flatness assumption, that this makes the Bökstedt spectral sequence converging to the mod $p$ homology of $\operatorname{THH}(R)$ into a Hopf algebra spectral sequence. We then apply this additional structure to the study of some interesting examples, including the commutative $S$-algebras $k u, k o, t m f, j u$ and $j$, and to calculate the homotopy groups of $T H H(k u)$ and $T H H(k o)$ after smashing with suitable finite complexes. This is part of a program to make systematic computations of the algebraic $K$-theory of $S$-algebras, by means of the cyclotomic trace map to topological cyclic homology.
\end{abstract}

AMS Classification 55P43, 55S10, 55S12, 57T05; 13D03, 55T15

Keywords Topological Hochschild homology, commutative $S$-algebra, coproduct, Hopf algebra, topological $K$-theory, image-of- $J$ spectrum, Bökstedt spectral sequence, Steenrod operations, Dyer-Lashof operations.

\section{Introduction}

The topological Hochschild homology $T H H(R)$ of an $S$-algebra $R$ (or an $A_{\infty}$ ring spectrum, or a functor with smash product) was constructed in the mid1980's by Bökstedt Bo1, as the natural promotion of the classical Hochschild homology of an algebra in the category of vector spaces (equipped with tensor product) to one in the category of spectra (equipped with smash product). It is the initial ingredient in the construction by Bökstedt, Hsiang and Madsen BHM93 of the topological cyclic homology $T C(R ; p)$, which in many cases closely approximates the algebraic $K$-theory $K(R)$ [Mc97, Du97, HM97.

When $R=H \mathcal{O}_{E}$ is the Eilenberg-Mac Lane spectrum of the valuation ring in a local number field, systematic computations of the topological cyclic homology of $R$ were made in [HM03, thereby verifying the Lichtenbaum-Quillen 
conjectures for the algebraic $K$-theory of these fields. Particular computations for other commutative $S$-algebras, like connective complex $K$-theory $k u$ and its $p$-local Adams summand $\ell$, have revealed a more general pattern of how algebraic $K$-theory creates a "red-shift" in chromatic filtration and satisfies a Galois descent property AR02, Au].

These results indicate that the algebraic $K$-theory of a commutative $S$-algebra is governed by an associated " $S$-algebro-geometric" structure space, for which the smashing localizations of the chromatic filtration are related to a Zariski topology, and étale covers and Galois extensions are related to an étale topology. There are also variant geometries associated to the less strictly commutative $S$ algebras known as $E_{n}$ ring spectra, for $1 \leq n \leq \infty$. The extreme cases $n=1$ and $n=\infty$ correspond to the associative and the commutative $S$-algebras, respectively. We shall encounter examples of the intermediate $E_{n}$ ring spectrum structures in Section 5, when we consider the Brown-Peterson spectrum BP (which is an $E_{4}$ ring spectrum according to Basterra and Mandell) and the Johnson-Wilson spectra $B P\langle m-1\rangle$. By convention, we let $B P=B P\langle\infty\rangle$.

Further systematic computations of the topological Hochschild homology, topological cyclic homology and algebraic $K$-theory of $S$-algebras can be expected to shed new light on these geometries and on the algebraic $K$-theory functor. The present paper advances the algebraic topological foundations for such systematic computations, especially by taking into account the Hopf algebra structure present in the topological Hochschild homology of commutative $S$ algebras. This program is continued in BR05, which analyzes the differentials in the homological homotopy fixed point spectral sequence that approximates the cyclic fixed points of topological Hochschild homology, and in L-N05, which identifies the action by Steenrod operations on the (continuous co-)homology of these fixed- and homotopy fixed point spectra.

When $R$ is a commutative $S$-algebra (or an $E_{\infty}$ ring spectrum, or perhaps a commutative FSP), there is an equivalence $T H H(R) \simeq R \otimes S^{1}$ of commutative $R$-algebras, due to McClure, Schwänzl and Vogt [MSV97, where $S^{1}$ is the topological circle and $(-) \otimes S^{1}$ refers to the topologically tensored structure in the category of commutative $S$-algebras. The pinch map $S^{1} \rightarrow S^{1} \vee S^{1}$ and reflection $S^{1} \rightarrow S^{1}$ then induce maps $\psi: T H H(R) \rightarrow T H H(R) \wedge_{R} T H H(R)$ and $\chi: T H H(R) \rightarrow T H H(R)$, which make $T H H(R)$ a Hopf algebra over $R$ in the homotopy category [EKMM97, IX.3.4]. See also Theorem 3.9.

We wish to apply this added structure for computations. Such computations are usually made by starting with the simplicial model $[q] \mapsto R^{\wedge(q+1)}=R \otimes S_{q}^{1}$ 
for $T H H(R)$, where now $[q] \mapsto S_{q}^{1}$ is the simplicial circle. The resulting skeleton filtration on $T H H(R)$ gives rise to the Bökstedt spectral sequence in homology

$$
E_{* *}^{2}(R)=H H_{*}\left(H_{*}\left(R ; \mathbb{F}_{p}\right)\right) \Longrightarrow H_{*}\left(T H H(R) ; \mathbb{F}_{p}\right),
$$

as we explain in Section 4. Compare [BM94, §2] and [MS93, 3.1]. But the coproduct and conjugation maps are not induced by simplicial maps in this model, so some adjustment is needed in order for these structures to carry over to the spectral sequence. This we arrange in Section 3, by using a doubly subdivided simplicial circle to provide an alternative simplicial model for $T H H(R)$, for which the Hopf algebra structure maps can be simplicially defined. The verification of the Hopf algebra relations then also involves a triply subdivided simplicial circle.

In Section 4 we transport the Hopf algebra structure on $\operatorname{THH}(R)$ to the Bökstedt spectral sequence, showing in Theorem 4.5 that if its initial term $E_{* *}^{2}(R)$ is flat as a module over $H_{*}\left(R ; \mathbb{F}_{p}\right)$, then this term is an $A_{*}$-comodule $H_{*}\left(R ; \mathbb{F}_{p}\right)$-Hopf algebra and the $d^{2}$-differentials respect this structure. Furthermore, if every $E^{r}$-term is flat over $H_{*}\left(R ; \mathbb{F}_{p}\right)$, then the Bökstedt spectral sequence is one of $A_{*}$-comodule $H_{*}\left(R ; \mathbb{F}_{p}\right)$-Hopf algebras. We also discuss, in Proposition 4.3, the weaker algebraic structure available in the Bökstedt spectral sequence when $R$ is just an $E_{2}$ - or $E_{3}$ ring spectrum.

Thereafter we turn to the computational applications. The mod $p$ homology of the topological Hochschild homology of the Eilenberg-Mac Lane $S$-algebras $H \mathbb{F}_{p}$ and $H \mathbb{Z}$ was already computed by Bökstedt Bo2 . The first non-algebraic example, namely the topological Hochschild homology of the Adams summand $\ell=B P\langle 1\rangle$ of $p$-local connective topological $K$-theory, was computed for $p$ odd by McClure and Staffeldt in MS93.

We show in Section 5 how to extend these computations to include the case of $k u_{(2)}=B P\langle 1\rangle$ at $p=2$, and the more general Brown-Peterson and JohnsonWilson $S$-algebras $B P\langle m-1\rangle$, for primes $p$ and symbols $0 \leq m \leq \infty$ such that these are $E_{3}$ ring spectra. As a sample result we have part of Theorem [5.12(a):

$$
H_{*}\left(T H H(B P) ; \mathbb{F}_{2}\right) \cong H_{*}\left(B P ; \mathbb{F}_{2}\right) \otimes E\left(\sigma \bar{\xi}_{k}^{2} \mid k \geq 1\right) .
$$

Here and below, $E(-)$ and $P(-)$ denote the exterior and polynomial algebras on the indicated generators, respectively, and the classes $\bar{\xi}_{k}$ are the conjugates of Milnor's generators for the dual Steenrod algebra $A_{*}$.

To resolve the multiplicative extensions in the Bökstedt spectral sequence we provide a proof of Bökstedt's formula saying that the suspension map $\sigma: \Sigma R \rightarrow$ 
THH $(R)$ takes the Dyer-Lashof operations $Q^{k}$ on the homology of the commutative $S$-algebra $R$ compatibly to the corresponding operations on the homology of the commutative $S$-algebra $T H H(R)$. See Proposition 5.9, which also makes precise what happens when $R$ is just an $E_{n+1}$ ring spectrum.

In Section 6 we do the same for the higher real commutative $S$-algebras $k o$ and tmf at $p=2$. As sample results we have Corollary 5.14(a) and Theorem 6.2(a):

$$
H_{*}\left(T H H(k u) ; \mathbb{F}_{2}\right) \cong H_{*}\left(k u ; \mathbb{F}_{2}\right) \otimes E\left(\sigma \bar{\xi}_{1}^{2}, \sigma \bar{\xi}_{2}^{2}\right) \otimes P\left(\sigma \bar{\xi}_{3}\right)
$$

and

$$
H_{*}\left(T H H(k o) ; \mathbb{F}_{2}\right) \cong H_{*}\left(k o ; \mathbb{F}_{2}\right) \otimes E\left(\sigma \bar{\xi}_{1}^{4}, \sigma \bar{\xi}_{2}^{2}\right) \otimes P\left(\sigma \bar{\xi}_{3}\right)
$$

In the more demanding Section 7 we proceed to the $p$-local real and complex image-of- $J$ spectra $j$ and $j u$, which are connective, commutative $S$-algebras. At odd primes the two are homotopy equivalent. We identify the mod $p$ homology algebra of $j u$ at $p=2$ and of $j=j u$ at odd primes in Proposition [7.12(a) and (b), and make essential use of our results about Hopf algebra structures to show that the corresponding Bökstedt spectral sequences for $T H H$ collapse at the $E^{2}$ - and $E^{p}$-terms, respectively, in Proposition 17.13(a) and (b). In Theorem 7.15 we resolve the algebra extension questions to obtain $H_{*}\left(T H H(j u) ; \mathbb{F}_{p}\right)$ as an algebra, both for $p=2$ and for $p$ odd. This proof involves a delicate comparison with the case of $T H H(k u)$ for $p=2$, and with $T H H(\ell)$ for $p$ odd. Again as a sample result, we have Theorem [7.15(a):

$$
H_{*}\left(T H H(j u) ; \mathbb{F}_{2}\right) \cong H_{*}\left(j u ; \mathbb{F}_{2}\right) \otimes E\left(\sigma \bar{\xi}_{1}^{4}, \sigma \bar{\xi}_{2}^{2}\right) \otimes P\left(\sigma \bar{\xi}_{3}\right) \otimes \Gamma(\sigma b) .
$$

Here $\Gamma(\sigma b)=E\left(\gamma_{2^{k}}(\sigma b) \mid k \geq 0\right)$ is the divided power algebra on a class $\sigma b$ in degree 4 .

The algebra structure of $H_{*}\left(j ; \mathbb{F}_{2}\right)$ is described as a split square-zero extension of $\left(A / / A_{2}\right)_{*}$ in Proposition [7.12(c):

$$
0 \rightarrow A_{*} \square_{A_{2 *}} \Sigma^{7} K_{*} \rightarrow H_{*}\left(j ; \mathbb{F}_{2}\right) \rightarrow\left(A / / A_{2}\right)_{*} \rightarrow 0 .
$$

Here $A_{2}=\left\langle S q^{1}, S q^{2}, S q^{4}\right\rangle \subset A$, and $K_{*} \subset A_{2 *}$ is dual to a cyclic $A_{2}$-module $K$ of rank 17 over $\mathbb{F}_{2}$. The symbol $\square$ denotes the cotensor product of comodules MiMo65, 2.2]. The $A$-module structure of $H^{*}\left(j ; \mathbb{F}_{2}\right)$ was given in Da75, but this identification of the algebra structure seems to be new. The $E^{2}$-term of the Bökstedt spectral sequence for $j$ is described in Proposition 7.13)(c), but it is not flat over $H_{*}\left(j ; \mathbb{F}_{2}\right)$, so the coproduct on $H_{*}\left(T H H(j) ; \mathbb{F}_{2}\right)$ is not conveniently described by this spectral sequence. We have therefore not managed to evaluate the homology of $T H H(j)$ at $p=2$ by these methods. 
Next we consider the passage from the homology of $T H H(R)$ to its homotopy, with suitably chosen finite coefficients. This has been a necessary technical switch in past computations of topological cyclic homology $T C(R ; p)$, since $T C$ is defined as the homotopy inverse limit of a diagram of fixed-point spectra derived from $\mathrm{THH}$, and the interaction between inverse limits and homology was thought to be difficult to control. The homotopy groups of an inverse limit are much better behaved. Nonetheless, it may be that future computations of the topological cyclic homology of $S$-algebras will follow a purely homological approach, see BR05] and [-N05.

In Section 8 we follow the strategy of MS93] to compute the homotopy groups of $T H H(k u) \wedge M$, where $M=C_{2}$ is the $\bmod 2$ Moore spectrum, and of $T H H(k o) \wedge Y$, where $Y=C_{2} \wedge C_{\eta}$ is the 4-cell spectrum employed by Mahowald Mah82. The results appear in Theorems 8.13 and 8.14, respectively. In each case the method is to use the Adams spectral sequence to pass from homology to homotopy, and to use a comparison with a Morava $K(1)$-based Bökstedt spectral sequence to obtain enough information about the $v_{1}$-periodic towers in the abutment to completely determine the differential structure of the Adams spectral sequence.

The present paper started out as the first author's Master's thesis An02 at the University of Oslo, supervised by the second author. Both authors are grateful to the referee for his careful reading of the paper.

\section{Hochschild and topological Hochschild homology}

Let $k$ be a graded field, i.e., a graded commutative ring such that every graded $k$-module is free, and $\Lambda$ a graded $k$-algebra (always unital and associative). We recall the definition of the Hochschild homology of $\Lambda$, e.g. from Mac75, X.4]. The Hochschild complex $C_{*}(\Lambda)=C_{*}^{k}(\Lambda)$ is the chain complex of graded $k$-modules with $C_{q}(\Lambda)=\Lambda^{\otimes(q+1)}$ in degree $q$ (all tensor products are over $k$ ) and boundary homomorphisms $\partial: C_{q}(\Lambda) \rightarrow C_{q-1}(\Lambda)$ given by

$$
\begin{aligned}
& \partial\left(\lambda_{0} \otimes \cdots \otimes \lambda_{q}\right)= \\
& \quad \sum_{i=0}^{q-1}(-1)^{i} \lambda_{0} \otimes \cdots \otimes \lambda_{i} \lambda_{i+1} \otimes \cdots \otimes \lambda_{q}+(-1)^{q+\epsilon} \lambda_{q} \lambda_{0} \otimes \cdots \otimes \lambda_{q-1}
\end{aligned}
$$

where $\epsilon=\left|\lambda_{q}\right|\left(\left|\lambda_{0}\right|+\cdots+\left|\lambda_{q-1}\right|\right)$. The Hochschild homology $H H_{*}(\Lambda)=$ $H H_{*}^{k}(\Lambda)$ is defined to be the homology of this chain complex. It is bigraded, 
first by the Hochschild degree $q$ and second by the internal grading from $\Lambda$. When $\Lambda$ is commutative (always in the graded sense) the shuffle product of chains defines a product

$$
\phi: H H_{*}(\Lambda) \otimes_{\Lambda} H H_{*}(\Lambda) \rightarrow H H_{*}(\Lambda)
$$

that makes $H H_{*}(\Lambda)$ a commutative $\Lambda$-algebra, with unit corresponding to the inclusion of 0-chains $\Lambda \rightarrow H H_{*}(\Lambda)$.

When $\Lambda$ is commutative there is also a chain level coproduct $\psi: C_{*}(\Lambda) \rightarrow$ $C_{*}(\Lambda) \otimes_{\Lambda} C_{*}(\Lambda)$ given in degree $q$ by

$$
\begin{aligned}
\psi\left(\lambda_{0} \otimes \lambda_{1} \otimes \cdots \otimes \lambda_{q}\right) & = \\
& \sum_{i=0}^{q}\left(\lambda_{0} \otimes \lambda_{1} \otimes \cdots \otimes \lambda_{i}\right) \otimes_{\Lambda}\left(1 \otimes \lambda_{i+1} \otimes \cdots \otimes \lambda_{q}\right) .
\end{aligned}
$$

It is essential to tensor over $\Lambda$ in the target of this chain map. When $H H_{*}(\Lambda)$ is flat as a $\Lambda$-module, the chain level coproduct $\psi$ induces a coproduct

$$
\psi: H H_{*}(\Lambda) \rightarrow H H_{*}(\Lambda) \otimes_{\Lambda} H H_{*}(\Lambda)
$$

on Hochschild homology. Here the right hand side is identified with the homology of $C_{*}(\Lambda) \otimes_{\Lambda} C_{*}(\Lambda)$ by the Künneth theorem. We shall now compare this chain level definition of the coproduct on $H H_{*}(\Lambda)$ with an equivalent definition given in more simplicial terms.

Let $B_{*}(\Lambda)=B_{*}(\Lambda, \Lambda, \Lambda)$ be the two-sided bar construction Mac75, X.2] for the $k$-algebra $\Lambda$. It has $B_{q}(\Lambda)=\Lambda \otimes \Lambda^{\otimes q} \otimes \Lambda$ in degree $q$, and is a free resolution of $\Lambda$ in the category of $\Lambda$-bimodules. We use the bar notation $\lambda_{0}\left[\lambda_{1}|\ldots| \lambda_{q}\right] \lambda_{q+1}$ for a typical generator of $B_{q}(\Lambda)$. The Hochschild complex is obtained from the two-sided bar construction by tensoring it with $\Lambda$ viewed as a $\Lambda$-bimodule: $C_{*}(\Lambda)=\Lambda \otimes_{\Lambda-\Lambda} B_{*}(\Lambda)$.

When $\Lambda$ is commutative, $B_{*}(\Lambda)$ is the chain complex $\operatorname{Ch}\left(\Lambda \otimes \Delta^{1}\right)$ associated to the simplicial $\Lambda$-bimodule $[q] \mapsto \Lambda \otimes \Delta_{q}^{1}$. Here $\Delta^{1}$ is the simplicial 1-simplex, and $\Lambda \otimes \Delta_{q}^{1}$ denotes the tensor product of one copy of $\Lambda$ for each element of $\Delta_{q}^{1}$. See Section 3 below for more on this notation. The $\Lambda$-bimodule structure on $B_{*}(\Lambda)$ is derived from the inclusion of the two boundary points $\partial \Delta^{1} \rightarrow \Delta^{1}$, and $C_{*}(\Lambda)$ equals the chain complex $\operatorname{Ch}\left(\Lambda \otimes S^{1}\right)$ associated to the simplicial $\Lambda$-module $[q] \mapsto \Lambda \otimes S_{q}^{1}$, where $S^{1}=\Delta^{1} / \partial \Delta^{1}$ is the simplicial circle.

We now discuss three maps of $\Lambda$-bimodule chain complexes. First, there is a canonical chain level coproduct $\psi: B_{*}(\Lambda) \rightarrow B_{*}(\Lambda) \otimes_{\Lambda} B_{*}(\Lambda)$ of $\Lambda$-bimodules, 
given in degree $q$ by

$$
\psi\left(\lambda_{0}\left[\lambda_{1}|\ldots| \lambda_{q}\right] \lambda_{q+1}\right)=\sum_{i=0}^{q} \lambda_{0}\left[\lambda_{1}|\ldots| \lambda_{i}\right] 1 \otimes_{\Lambda} 1\left[\lambda_{i+1}|\ldots| \lambda_{q}\right] \lambda_{q+1} .
$$

When $\Lambda$ is commutative the chain level coproduct $\psi$ on $C_{*}(\Lambda)$ is derived from this, as the obvious composite map

$$
\begin{gathered}
C_{*}(\Lambda)=\Lambda \otimes_{\Lambda-\Lambda} B_{*}(\Lambda) \stackrel{1 \otimes \psi}{\longrightarrow} \Lambda \otimes_{\Lambda-\Lambda}\left(B_{*}(\Lambda) \otimes_{\Lambda} B_{*}(\Lambda)\right) \\
C_{*}(\Lambda) \otimes_{\Lambda}^{\prime} C_{*}(\Lambda) .
\end{gathered}
$$

Second, there is a shuffle equivalence $s h: B_{*}(\Lambda) \otimes_{\Lambda} B_{*}(\Lambda) \rightarrow d B_{*}(\Lambda)$ of $\Lambda$ bimodules, by the Eilenberg-Zilber theorem [Mac75, VIII.8.8] applied to two copies of the simplicial $\Lambda$-bimodule $\Lambda \otimes \Delta^{1}$. Here

$$
d B_{*}(\Lambda)=\operatorname{Ch}\left(\Lambda \otimes d \Delta^{1}\right)=\operatorname{Ch}\left(\left(\Lambda \otimes \Delta^{1}\right) \otimes_{\Lambda}\left(\Lambda \otimes \Delta^{1}\right)\right)
$$

is the chain complex associated to the simplicial tensor product of two copies of $\Lambda \otimes \Delta^{1}$, considered as simplicial $\Lambda$-modules by way of the right and left actions, respectively. This simplicial tensor product equals $\Lambda \otimes d \Delta^{1}$, where the "double 1-simplex" $d \Delta^{1}=\Delta^{1} \cup_{\Delta^{0}} \Delta^{1}$ is the union of two 1-simplices that are compatibly oriented. (So $d \Delta^{1}$ is the 2 -fold edgewise subdivision of $\Delta^{1}$ BHM93, §1].) More explicitly,

$$
\operatorname{sh}\left(x \otimes_{\Lambda} y\right)=\sum_{(\mu, \nu)} \operatorname{sgn}(\mu, \nu)\left(s_{\nu}(x) \otimes_{\Lambda} s_{\mu}(y)\right),
$$

where $x \in B_{i}(\Lambda), y \in B_{q-i}(\Lambda)$, the sum is taken over all $(i, q-i)$-shuffles $(\mu, \nu), \operatorname{sgn}(\mu, \nu)$ is the sign of the associated permutation and $s_{\nu}(x)$ and $s_{\mu}(y)$ are the appropriate iterated degeneracy operations on $x$ and $y$, respectively.

Third, there is a chain equivalence $\pi: d B_{*}(\Lambda) \rightarrow B_{*}(\Lambda)$ of $\Lambda$-bimodules, induced by the simplicial map $\pi: d \Delta^{1}=\Delta^{1} \cup_{\Delta^{0}} \Delta^{1} \rightarrow \Delta^{1}$ that collapses the second $\Delta^{1}$ in $d \Delta^{1}$ to a point. It is given by

$$
\pi\left(x \otimes_{\Lambda} y\right)=x \cdot \epsilon(y)
$$

for $x, y \in B_{q}(\Lambda)$, where $\epsilon\left(\lambda_{0}\left[\lambda_{1}|\ldots| \lambda_{q}\right] \lambda_{q+1}\right)=\lambda_{0} \lambda_{1} \ldots \lambda_{q} \lambda_{q+1}$ is the augmentation.

Lemma 2.2 Let $\Lambda$ be a commutative $k$-algebra. The maps $s h \circ \psi: B_{*}(\Lambda) \rightarrow$ $d B_{*}(\Lambda)$ and $\pi: d B_{*}(\Lambda) \rightarrow B_{*}(\Lambda)$ of $\Lambda$-bimodule chain complexes are mutual chain inverses. Hence the induced composite

$$
d C_{*}(\Lambda)=\Lambda \otimes_{\Lambda-\Lambda} d B_{*}(\Lambda) \stackrel{1 \otimes \pi}{\longrightarrow} C_{*}(\Lambda) \stackrel{1 \otimes(s h \circ \psi)}{\longrightarrow} d C_{*}(\Lambda)
$$


is chain homotopic to the identity.

Proof All three chain complexes $B_{*}(\Lambda), B_{*}(\Lambda) \otimes_{\Lambda} B_{*}(\Lambda)$ and $d B_{*}(\Lambda)$ are free $\Lambda$-bimodule resolutions of $\Lambda$, and the maps $\psi$, sh and $\pi$ are $\Lambda$-bimodule chain maps, so it suffices to verify that the composite $\pi \circ s h \circ \psi: B_{*}(\Lambda) \rightarrow$ $B_{*}(\Lambda)$ covers the identity on $\Lambda$. In degree zero, $\psi\left(\lambda_{0}[] \lambda_{1}\right)=\lambda_{0}[] 1 \otimes_{\Lambda} 1[] \lambda_{1}$, $\operatorname{sh}\left(\lambda_{0}[] 1 \otimes_{\Lambda} 1[] \lambda_{1}\right)=\lambda_{0}[] 1 \otimes_{\Lambda} 1[] \lambda_{1}$ and $\pi\left(\lambda_{0}[] 1 \otimes_{\Lambda} 1[] \lambda_{1}\right)=\lambda_{0}[] \lambda_{1}$, as required. If desired, the explicit formulas can be composed also in higher degrees, to show that $(\pi \circ s h \circ \psi)(x) \equiv x$ modulo simplicially degenerate terms, for all $x \in B_{q}(\Lambda)$. In either case, we see that $\pi \circ s h \circ \psi$ is chain homotopic to the identity. The remaining conclusions follow by uniqueness of inverses.

Note that $d C_{*}(\Lambda)$ defined above is the chain complex associated to the simplicial $\Lambda$-module $\Lambda \otimes d^{\prime} S^{1}$, where the "double circle" $d^{\prime} S^{1}=d \Delta^{1} / \partial d \Delta^{1}$ is the quotient of the double 1-simplex $d \Delta^{1}=\Delta^{1} \cup_{\Delta^{0}} \Delta^{1}$ by its two end-points $\partial d \Delta^{1}$. The chain equivalence $1 \otimes \pi: d C_{*}(\Lambda)=\operatorname{Ch}\left(\Lambda \otimes d^{\prime} S^{1}\right) \rightarrow \operatorname{Ch}\left(\Lambda \otimes S^{1}\right)=C_{*}(\Lambda)$ obtained by tensoring down the $\Lambda$-bimodule chain equivalence induced from the collapse map $\pi: d \Delta^{1} \rightarrow \Delta^{1}$, is then more directly obtained from the collapse map $\pi: d^{\prime} S^{1} \rightarrow S^{1}$ that collapses the second of the two non-degenerate 1simplices in $d^{\prime} S^{1}$ to a point.

There is also a simplicial pinch map $\psi: d^{\prime} S^{1} \rightarrow S^{1} \vee S^{1}$ to the one-point union (wedge sum) of two circles, that collapses the 0 -skeleton of $d^{\prime} S^{1}$ to a point. It induces a map

$$
\psi^{\prime}: d C_{*}(\Lambda)=\mathrm{Ch}\left(\Lambda \otimes d^{\prime} S^{1}\right) \rightarrow \operatorname{Ch}\left(\Lambda \otimes\left(S^{1} \vee S^{1}\right)\right) .
$$

The target is the chain complex associated to the simplicial tensor product of two copies of $\Lambda \otimes S^{1}$, considered as a simplicial $\Lambda$-module. It is therefore also the target of a shuffle equivalence, namely $s h: C_{*}(\Lambda) \otimes_{\Lambda} C_{*}(\Lambda) \rightarrow \operatorname{Ch}\left(\Lambda \otimes\left(S^{1} \vee S^{1}\right)\right)$.

Proposition 2.3 Let $\Lambda$ be a commutative $k$-algebra. The composite map

$$
d C_{*}(\Lambda) \stackrel{1 \otimes \pi}{\simeq} C_{*}(\Lambda) \stackrel{\psi}{\longrightarrow} C_{*}(\Lambda) \otimes_{\Lambda} C_{*}(\Lambda) \stackrel{\text { sh }}{\simeq} \mathrm{Ch}\left(\Lambda \otimes\left(S^{1} \vee S^{1}\right)\right)
$$

of the chain level coproduct $\psi$ in $C_{*}(\Lambda)$ (see formula (2.1)), with the chain equivalence $1 \otimes \pi$ induced by the simplicial collapse map $\pi: d^{\prime} S^{1} \rightarrow S^{1}$ and the shuffle equivalence $s h$, is chain homotopic to the map

$$
d C_{*}(\Lambda)=\operatorname{Ch}\left(\Lambda \otimes d^{\prime} S^{1}\right) \stackrel{\psi^{\prime}}{\longrightarrow} \operatorname{Ch}\left(\Lambda \otimes\left(S^{1} \vee S^{1}\right)\right)
$$

induced by the simplicial pinch map $\psi: d^{\prime} S^{1} \rightarrow S^{1} \vee S^{1}$. 
Hence, when $H_{*}(\Lambda)$ is flat over $\Lambda$, the coproduct $\psi$ on Hochschild homology agrees, via the identifications induced by $1 \otimes \pi$ and $s h$, with the map $\psi^{\prime}$ induced by the simplicial pinch map.

Proof Consider the following diagram.

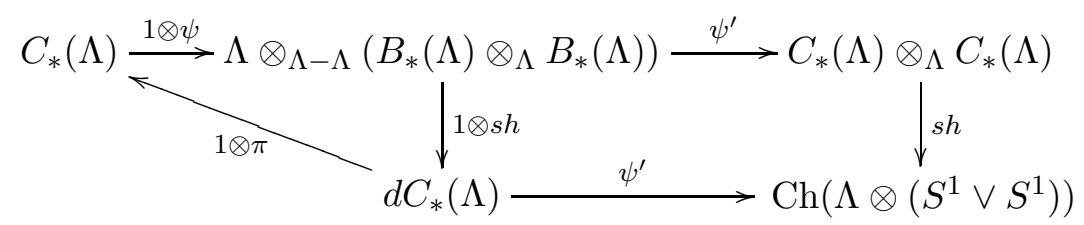

The composite along the upper row is the coproduct $\psi$, the composite around the triangle is chain homotopic to the identity by Lemma 2.2. and the square commutes by naturality of the shuffle map with respect to the pinch map $\psi^{\prime}$. A diagram chase then provides the claimed chain homotopy.

We shall make use of the following standard calculations of Hochschild homology. The formulas for the coproduct $\psi$ follow directly from the chain level formula (2.1) above. Let $P(x)=k[x]$ and $E(x)=k[x] /\left(x^{2}\right)$ be the polynomial and exterior algebras over $k$ in one variable $x$, and let $\Gamma(x)=k\left\{\gamma_{i}(x) \mid i \geq 0\right\}$ be the divided power algebra with multiplication

$$
\gamma_{i}(x) \cdot \gamma_{j}(x)=(i, j) \gamma_{i+j}(x),
$$

where $(i, j)=(i+j) ! / i ! j$ ! is the binomial coefficient.

Proposition 2.4 For $x \in \Lambda$ let $\sigma x \in H H_{1}(\Lambda)$ be the homology class of the cycle $1 \otimes x \in C_{1}(\Lambda)$ in the Hochschild complex. For $\Lambda=P(x)$ there is a $P(x)$-algebra isomorphism

$$
H H_{*}(P(x))=P(x) \otimes E(\sigma x) .
$$

The class $\sigma x$ is coalgebra primitive, i.e., $\psi(\sigma x)=\sigma x \otimes 1+1 \otimes \sigma x$. For $\Lambda=E(x)$ there is an $E(x)$-algebra isomorphism

$$
H H_{*}(E(x))=E(x) \otimes \Gamma(\sigma x) .
$$

The $i$ th divided power $\gamma_{i}(\sigma x)$ is the homology class of the cycle $1 \otimes x \otimes \cdots \otimes x \in$ $C_{i}(\Lambda)$. The coproduct is given by

$$
\psi\left(\gamma_{k}(\sigma x)\right)=\sum_{i+j=k} \gamma_{i}(\sigma x) \otimes \gamma_{j}(\sigma x) .
$$

There is a Künneth formula

$$
H H_{*}\left(\Lambda_{1} \otimes \Lambda_{2}\right) \cong H H_{*}\left(\Lambda_{1}\right) \otimes H H_{*}\left(\Lambda_{2}\right) .
$$


Let $P_{h}(x)=k[x] /\left(x^{h}\right)$ be the truncated polynomial algebra of height $h$. We write $P\left(x_{i} \mid i \geq 0\right)=P\left(x_{0}, x_{1}, \ldots\right)=P\left(x_{0}\right) \otimes P\left(x_{1}\right) \otimes \ldots$, and so on. When $k$ is of prime characteristic $p$ it is a standard calculation with binomial coefficients that

$$
\Gamma(x)=P_{p}\left(\gamma_{p^{i}}(x) \mid i \geq 0\right)
$$

as a $k$-algebra.

We have already noted that the Hochschild complex $C_{*}(\Lambda)$ is the chain complex associated to a simplicial graded $k$-module $[q] \mapsto C_{q}(\Lambda)=\Lambda^{\otimes(q+1)}$, with face maps $d_{i}$ corresponding to the individual terms in the alternating sum defining the Hochschild boundary $\partial$. In fact, this is a cyclic graded $k$-module in the sense of Connes, with cyclic structure maps $t_{q}$ that cyclically permute the $(q+1)$ tensor factors in $C_{q}(\Lambda)$, up to sign. It follows that the geometric realization $H H(\Lambda)=\left|[q] \mapsto C_{q}(\Lambda)\right|$ admits a natural $S^{1}$-action $\alpha: H H(\Lambda) \wedge S_{+}^{1} \rightarrow H H(\Lambda)$, and that the Hochschild homology groups are the homotopy groups of this space: $H H_{*}(\Lambda)=\pi_{*} H H(\Lambda)$.

The basic idea in the definition of topological Hochschild homology is to replace the ground ring $k$ by the sphere spectrum $S$, and the symmetric monoidal category of graded $k$-modules under the tensor product $\otimes=\otimes_{k}$ by the symmetric monoidal category of spectra, interpreted as $S$-modules, under the smash product $\wedge=\wedge_{S}$. A monoid in the first category is a graded $k$-algebra $\Lambda$, which then gets replaced by a monoid in the second category, i.e., an $S$-algebra $R$. To make sense of this we will work in the framework of [EKMM97, but we could also use HSS00 or any other reasonable setting that gives a symmetric monoidal category of spectra.

The original definition of topological Hochschild homology was given by Bökstedt in the mid 1980's Bo1, inspired by work and conjectures of Goodwillie and Waldhausen. The following definition is not the one originally used by Bökstedt, since he did not have the symmetric monoidal smash product from [EKMM97 or [HSS00] available, but it agrees with the heuristic definition that his more complicated definition managed to make sense of, with the more elementary technology that he had at hand.

Definition 2.6 Let $R$ be an $S$-algebra, with multiplication $\mu: R \wedge R \rightarrow R$ and unit $\eta: S \rightarrow R$. The topological Hochschild homology of $R$ is the geometric realization $T H H(R)$ of the simplicial $S$-module $T H H \bullet(R)$ with

$$
\operatorname{THH}_{q}(R)=R^{\wedge(q+1)}
$$


in simplicial degree $q$. The simplicial structure is like that on the simplicial $k$-module underlying the Hochschild complex. More precisely, the $i$-th face map $d_{i}: R^{\wedge(q+1)} \rightarrow R^{\wedge q}$ equals $i d_{R}^{i} \wedge \mu \wedge i d_{R}^{q-i-1}$ for $0 \leq i<q$, while $d_{q}=$ $\left(\mu \wedge i d_{R}^{q-1}\right) t_{q}$, where $t_{q}$ cyclically permutes the $(q+1)$ smash factors $R$ by moving the last factor to the front. The $j$-th degeneracy map $s_{j}: R^{\wedge(q+1)} \rightarrow R^{\wedge(q+2)}$ equals $i d_{R}^{j+1} \wedge \eta \wedge i d_{R}^{q-j}$, for $0 \leq j \leq q$.

Furthermore, the cyclic operators $t_{q}: \mathrm{THH}_{q}(R) \rightarrow T H H_{q}(R)$ make $T H H_{\bullet}(R)$ a cyclic $S$-module, so that its geometric realization has a natural $S^{1}$-action

$$
\alpha: T H H(R) \wedge S_{+}^{1} \rightarrow \operatorname{THH}(R) .
$$

The topological Hochschild homology groups of $R$ are defined to be the homotopy groups $\pi_{*} T H H(R)$.

When $R$ is a commutative $S$-algebra there is a product on $\operatorname{THH}(R)$ that makes it an augmented commutative $R$-algebra, with unit corresponding to the inclusion of 0 -simplices $R \rightarrow T H H(R)$. See (3.3) below. There is a discussion of alternative definitions of $T H H(R)$ in [EKMM97, IX].

\section{$3 \quad$ Hopf algebra structure on $\mathrm{THH}$}

Already Bökstedt noted that the simplicial structure on $T H H(R)$, as defined above, is derived from the simplicial structure on the standard simplicial circle $S^{1}=\Delta^{1} / \partial \Delta^{1}$. This can be made most precise in the case when $R$ is a commutative $S$-algebra, in which case there is a formula $T H H(R) \cong R \otimes S^{1}$ in terms of the simplicial tensor structure on the category of commutative $S$-algebras. A corresponding formula $T H H(R) \simeq R \otimes\left|S^{1}\right|$ in terms of the topological tensor structure was discussed by McClure, Schwänzl and Vogt in MSV97. We shall stick to the simplicial context, since we will make use of the resulting skeletal filtrations to form spectral sequences.

We now make this "tensored structure" explicit. Let $R$ be a commutative $S$-algebra and $X$ a finite set, and let

$$
R \otimes X=\bigwedge_{x \in X} R
$$

be the smash product of one copy of $R$ for each element of $X$. It is again a commutative $S$-algebra. Now let $f: X \rightarrow Y$ be a function between finite sets, 
and let $R \otimes f: R \otimes X \rightarrow R \otimes Y$ be the smash product over all $y \in Y$ of the maps

$$
R \otimes f^{-1}(y)=\bigwedge_{x \in f^{-1}(y)} R \rightarrow R=R \otimes\{y\}
$$

that are given by the iterated multiplication from the $\# f^{-1}(y)$ copies of $R$ on the left to the single copy of $R$ on the right. If $f^{-1}(y)$ is empty, this is by definition the unit map $\eta: S \rightarrow R$. Since $R$ is commutative, there is no ambiguity in how these iterated multiplications are to be formed.

Note that the construction $R \otimes X$ is functorial in both $R$ and $X$. Given an injection $X \rightarrow Y$ and any function $X \rightarrow Z$, there is a natural isomorphism

$$
R \otimes\left(Y \cup_{X} Z\right) \cong(R \otimes Y) \wedge_{(R \otimes X)}(R \otimes Z) .
$$

There is also a natural map

$$
R \wedge X_{+}=\bigvee_{x \in X} R \rightarrow \bigwedge_{x \in X} R=R \otimes X
$$

whose restriction to the wedge summand indexed by $x \in X$ is $R \otimes i_{x}$, where $i_{x}:\{x\} \rightarrow X$ is the inclusion.

By naturality, these constructions all extend degreewise to simplicial finite sets $X:[q] \mapsto X_{q}$, simplicial maps $f: X \rightarrow Y$, etc. In particular, we can define the simplicial commutative $S$-algebra

$$
R \otimes X=\left([q] \mapsto R \otimes X_{q}\right)
$$

with structure maps $R \otimes d_{i}, R \otimes s_{j}$, etc. There is then a useful natural map

$$
\omega: R \wedge X_{+} \rightarrow R \otimes X
$$

Consider the special case $X=S^{1}=\Delta^{1} / \partial \Delta^{1}$. Here $\Delta_{q}^{1}$ has $(q+2)$ elements $\left\{x_{0}, \ldots, x_{q+1}\right\}$ where $x_{t}:[q] \rightarrow[1]$ has $\# x_{t}^{-1}(0)=t$. The quotient $S_{q}^{1}$ has $(q+1)$ elements, obtained by identifying $x_{0} \sim x_{q+1}$. Then $d_{i}\left(x_{t}\right)=x_{t}$ for $t \leq i$ and $d_{i}\left(x_{t}\right)=x_{t-1}$ for $t>i$, while $s_{j}\left(x_{t}\right)=x_{t}$ for $t \leq j$ and $s_{j}\left(x_{t}\right)=x_{t+1}$ for $t>j$. A direct check shows that there is a natural isomorphism

$$
T H H(R) \cong R \otimes S^{1}
$$

of (simplicial) commutative $S$-algebras. In degree $q$ it is the obvious identification $R^{\wedge(q+1)} \cong R \otimes S_{q}^{1}$.

There are natural maps $\eta: * \rightarrow S^{1}, \epsilon: S^{1} \rightarrow *$ and $\phi: S^{1} \vee S^{1} \rightarrow S^{1}$ that map to the base point of $S^{1}$, retract to $*$ and fold two copies of $S^{1}$ to one, 
respectively. By naturality, these induce the following maps of commutative $S$-algebras:

$$
\begin{aligned}
\eta & : R \rightarrow T H H(R) \\
\epsilon & : T H H(R) \rightarrow R \\
\phi & : T H H(R) \wedge_{R} \operatorname{THH}(R) \rightarrow T H H(R) .
\end{aligned}
$$

In the last case, the product map involves the identification

$$
T H H(R) \wedge_{R} T H H(R) \cong R \otimes\left(S^{1} \cup_{*} S^{1}\right),
$$

where $S^{1} \cup_{*} S^{1}=S^{1} \vee S^{1}$. Taken together, these maps naturally make $T H H(R)$ an augmented commutative $R$-algebra.

There is also a natural map

$$
\omega: R \wedge S_{+}^{1} \rightarrow T H H(R),
$$

derived from (3.1), which captures part of the circle action upon $T H H(R)$. More precisely, the map $\omega$ admits the following factorization:

$$
\omega=\alpha \circ(\eta \wedge i d): R \wedge S_{+}^{1} \rightarrow T H H(R) \wedge S_{+}^{1} \rightarrow T H H(R) .
$$

This is clear by inspection of the definition of the circle action $\alpha$ on the 0 simplices of $T H H(R)$.

We would like to have a coproduct on $\operatorname{THH}(R)$, coming from a pinch map $S^{1} \rightarrow S^{1} \vee S^{1}$, but there is no such simplicial map with our basic model for $S^{1}$. To fix this we again consider a "double model" for $S^{1}$, denoted $d S^{1}$, with

$$
d S^{1}=\left(\Delta^{1} \sqcup \Delta^{1}\right) \cup_{\left(\partial \Delta^{1} \sqcup \partial \Delta^{1}\right)} \partial \Delta^{1} .
$$

Here $\partial \Delta^{1} \sqcup \partial \Delta^{1} \rightarrow \partial \Delta^{1}$ is the identity map on each summand, so the two nondegenerate 1-simplices of $d S^{1}$ have opposing orientations in the geometrically realized circle. It is the quotient of the barycentric subdivision of $\Delta^{1}$ by its boundary.

Then we have a simplicial pinch map $\psi: d S^{1} \rightarrow S^{1} \vee S^{1}$ that collapses $\partial \Delta^{1} \subset$ $d S^{1}$ to $*$, as well as a simplicial flip map $\chi: d S^{1} \rightarrow d S^{1}$ that interchanges the two copies of $\Delta^{1}$.

Remark 3.6 The simplicial set $d S^{1}$ introduced here differs from the double circle $d^{\prime} S^{1}$ considered in Section 2, in that the orientation of the second 1simplex has been reversed. The switch is necessary here to make the flip map $\chi$ simplicial. In principle, we could have used the same $d S^{1}$ in Section 2 as here, but this would have entailed the cost of discussing the anti-simplicial involution $\lambda_{0}\left[\lambda_{1}|\ldots| \lambda_{q}\right] \lambda_{q+1} \mapsto \pm \lambda_{q+1}\left[\lambda_{q}|\ldots| \lambda_{1}\right] \lambda_{0}$ of $B_{*}(\Lambda)$, and complicating the formula (2.1) for the chain level coproduct $\psi$. We choose instead to suppress this point. 
We define a corresponding "double model" for $T H H(R)$, denoted $d T H H(R)$, by

$$
d T H H(R)=R \otimes d S^{1} .
$$

The pinch and flip maps now induce the following natural maps of commutative $S$-algebras:

$$
\begin{aligned}
& \psi^{\prime}: d \operatorname{THH}(R) \rightarrow \operatorname{THH}(R) \wedge_{R} \operatorname{THH}(R) \\
& \chi^{\prime}: d \operatorname{THH}(R) \rightarrow d \operatorname{THH}(R) .
\end{aligned}
$$

Lemma 3.8 Let $R$ be cofibrant as an $S$-module. Then the collapse map $\pi: d S^{1} \rightarrow S^{1}$ that takes the second $\Delta^{1}$ to $*$ induces a weak equivalence

$$
\pi: \operatorname{dTHH}(R) \stackrel{\simeq}{\longrightarrow} \operatorname{THH}(R) .
$$

Proof Consider the commutative diagram

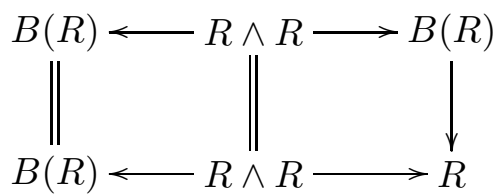

of commutative $S$-algebras. Here $B(R)=B(R, R, R)=R \otimes \Delta^{1}$ is the twosided bar construction, its augmentation $B(R) \rightarrow R$ is a weak equivalence, and the inclusion $R \wedge R \rightarrow B(R)$ is a cofibration of $S$-modules. From EKMM97, III.3.8] we know that the categorical pushout (balanced smash product) in this case preserves weak equivalences. Pushout along the upper row gives $d T H H(R)$ and pushout along the lower row gives $T H H(R)$, so the induced map $\pi: d T H H(R) \rightarrow T H H(R)$ is indeed a weak equivalence.

Theorem 3.9 Let $R$ be a commutative $S$-algebra. Its topological Hochschild homology $T H H(R)$ is naturally an augmented commutative $R$-algebra, with unit, counit and product maps $\eta, \epsilon$ and $\phi$ defined as in (3.3) above. In the stable homotopy category, these maps, the coproduct map

$$
\psi=\psi^{\prime} \circ \pi^{-1}: \operatorname{THH}(R) \rightarrow \operatorname{THH}(R) \wedge_{R} T H H(R)
$$

and the conjugation map

$$
\chi=\pi \circ \chi^{\prime} \circ \pi^{-1}: \operatorname{THH}(R) \rightarrow \operatorname{THH}(R)
$$

naturally make $T H H(R)$ an $R$-Hopf algebra. 
Proof To check that $T H H(R)$ is indeed a Hopf algebra over $R$ in the stable homotopy category we must verify that a number of diagrams commute. We will do one case that illustrates the technique, and leave the rest to the reader.

Let $T=T H H(R)$. In order to show that the diagram

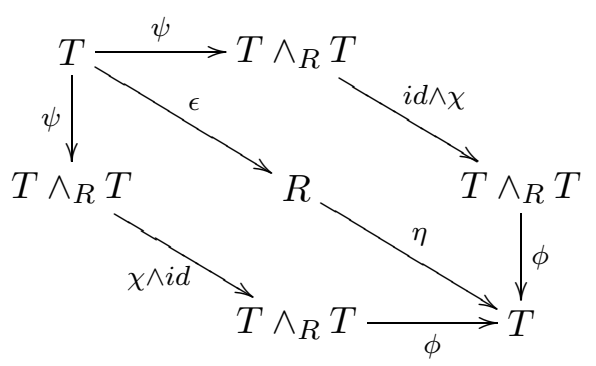

commutes in the stable homotopy category it suffices to check that the diagram of simplicial sets

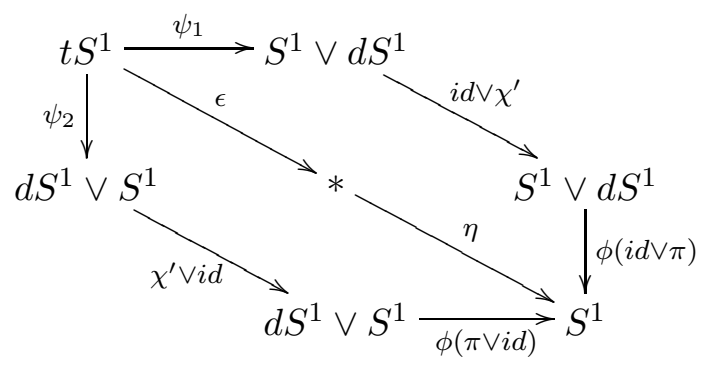

homotopy commutes (simplicially).

Here $t S^{1}=\partial \Delta^{2}$ is a "triple model" for $S^{1}$, with three non-degenerate 1simplices. The pinch map $\psi_{1}$ identifies the vertices 0 and 1 in $\partial \Delta^{2}$ and takes the face $\delta_{0}$ to the first $\Delta^{1}$ in $d S^{1}$. Then the composite $\phi(i d \vee \pi)\left(i d \vee \chi^{\prime}\right) \psi_{1}$ factors as

$$
\partial \Delta^{2} \subset \Delta^{2} \stackrel{s_{1}}{\longrightarrow} \Delta^{1} \rightarrow S^{1}
$$

and $\Delta^{1}$ is simplicially contractible. Similarly, $\psi_{2}$ identifies the vertices 1 and 2 and takes $\delta_{2}$ to the first $\Delta^{1}$ in $d S^{1}$. Then $\phi(\pi \vee i d)\left(\chi^{\prime} \vee i d\right) \psi_{2}$ factors as

$$
\partial \Delta^{2} \subset \Delta^{2} \stackrel{s_{0}}{\longrightarrow} \Delta^{1} \rightarrow S^{1}
$$

and again this map is simplicially contractible.

Finally we use a weak equivalence $t T H H(R)=R \otimes t S^{1} \rightarrow T H H(R)$, as in Lemma 3.8, to deduce that the diagram (3.10) indeed homotopy commutes. 
Remark 3.11 As noted above, $T H H(R)$ is commutative as an $R$-algebra. The pinch map $\psi: d S^{1} \rightarrow S^{1} \vee S^{1}$ is not homotopy cocommutative, so we do not expect that the coproduct $\psi: T H H(R) \rightarrow T H H(R) \wedge_{R} T H H(R)$ will be cocommutative in any great generality. The Hopf algebras arising in this fashion will therefore be commutative, but not cocommutative.

The inclusion of the base point $\eta: * \rightarrow S^{1}$ induces a cofiber sequence of $R$ modules

$$
R=R \wedge *_{+} \stackrel{1 \wedge \eta_{+}}{\longrightarrow} R \wedge S_{+}^{1} \stackrel{j}{\longrightarrow} R \wedge S^{1}=\Sigma R
$$

which is canonically split by the retraction map

$$
R \wedge S_{+}^{1} \stackrel{1 \wedge \epsilon_{+}}{\longrightarrow} R \wedge *_{+}=R .
$$

Hence in the stable homotopy category there is a canonical section $\kappa: \Sigma R \rightarrow$ $R \wedge S_{+}^{1}$ to the map labeled $j$ above. We let

$$
\sigma=\omega \circ \kappa: \Sigma R \rightarrow R \wedge S_{+}^{1} \rightarrow \operatorname{THH}(R)
$$

be the composite stable map. It induces an operator

$$
\sigma: H_{*}\left(\Sigma R ; \mathbb{F}_{p}\right) \rightarrow H_{*}\left(T H H(R) ; \mathbb{F}_{p}\right),
$$

which we in Proposition 4.9] shall see is compatible with that of Proposition 2.4

\section{The Bökstedt spectral sequence}

Let $R$ be an $S$-algebra. To calculate the $\bmod p$ homology $H_{*}\left(T H H(R) ; \mathbb{F}_{p}\right)$ of its topological Hochschild homology, Bökstedt constructed a strongly convergent spectral sequence

$$
E_{s, *}^{2}(R)=H H_{s}\left(H_{*}\left(R ; \mathbb{F}_{p}\right)\right) \Longrightarrow H_{s+*}\left(T H H(R) ; \mathbb{F}_{p}\right),
$$

using the skeleton filtration on $T H H(R)$. In fact,

$$
E_{s, *}^{1}(R)=H_{*}\left(R^{\wedge(s+1)} ; \mathbb{F}_{p}\right) \cong H_{*}\left(R ; \mathbb{F}_{p}\right)^{\otimes(s+1)}=C_{s}\left(H_{*}\left(R ; \mathbb{F}_{p}\right)\right)
$$

equals the Hochschild $s$-chains of the algebra $\Lambda=H_{*}\left(R ; \mathbb{F}_{p}\right)$ over $k=\mathbb{F}_{p}$, and the $d^{1}$-differential can as usual be identified with the Hochschild boundary operator $\partial$. To be quite precise, the $E^{1}$-term is really the associated normalized complex $\Lambda \otimes \bar{\Lambda}^{\otimes s}$, with $\bar{\Lambda}=\Lambda / k$, but this change does not affect the $E^{2}$-term.

This is naturally a spectral sequence of $A_{*}$-comodules, where $A_{*}=H_{*}\left(H \mathbb{F}_{p} ; \mathbb{F}_{p}\right)$ is the dual of the mod $p$ Steenrod algebra, since it is obtained by applying $\bmod p$ homology to a filtered spectrum. If $R$ is a commutative $S$-algebra, the spectral sequence admits more structure. 
Proposition 4.2 Let $R$ be a commutative $S$-algebra. Then $H_{*}\left(T H H(R) ; \mathbb{F}_{p}\right)$ is an augmented commutative $A_{*}$-comodule $H_{*}\left(R ; \mathbb{F}_{p}\right)$-algebra, and the Bökstedt spectral sequence $E_{* *}^{r}(R)$ is an augmented commutative $A_{*}$-comodule $H_{*}\left(R ; \mathbb{F}_{p}\right)$-algebra spectral sequence, converging to $H_{*}\left(T H H(R) ; \mathbb{F}_{p}\right)$.

Proof We know that $T H H(R)$ is an augmented commutative $R$-algebra by Theorem [3.9, and the relevant structure maps (3.3) are all maps of simplicial spectra. Hence they respect the skeleton filtration on $T H H(R)$, and we have in particular a composite map of spectral sequences

$$
E_{* *}^{r}(R) \otimes_{\Lambda} E_{* *}^{r}(R) \rightarrow^{\prime} E_{* *}^{r} \stackrel{\phi}{\longrightarrow} E_{* *}^{r}(R)
$$

with ' $E_{* *}^{r}$ the spectral sequence associated to the skeleton filtration on the smash product $T H H(R) \wedge_{R} T H H(R)$. The left hand map is induced by the usual homology cross product from the $E^{1}$-term and onwards. This defines the algebra structure on $E_{* *}^{r}(R)$, and the remaining claims are straightforward.

In fact, we can settle for less than strict commutativity to have an algebra structure in the Bökstedt spectral sequence. We shall make use of this for some examples related to the Brown-Peterson spectrum in Section 5. An $E_{n}$ ring spectrum [BMMS86, I.4] is an $S$-module with an action by the little $n$ cubes operad $\mathcal{C}_{n}$, or one that is related to it by a chain of $\Sigma$-equivariant weak equivalences. Fiedorowicz and Vogt [FV] 3.4] show that any $E_{n+1}$ ring spectrum is weakly equivalent to an associative $S$-algebra with an action by the little $n$ cubes operads, i.e., to a $\mathcal{C}_{n}$-algebra in the category of associative $S$-algebras. Basterra and Mandell (unpublished) have obtained the same result by a different method.

Thus any continuous functor from associative $S$-algebras takes an $E_{n+1}$ ring spectrum to a $\mathcal{C}_{n}$-algebra in its target category. This applies, for example, to the functor $T H H \bullet(-)$ from associative $S$-algebras to cyclic $S$-modules, and to its geometric realization $T H H(-)$ from associative $S$-algebras to $S^{1}$-equivariant $S$-modules. Therefore $T H H$ of an $E_{n+1}$ ring spectrum $R$ is an $S^{1}$-equivariant $E_{n}$ ring spectrum, and the unit $\eta: R \rightarrow T H H(R)$ is a map of $E_{n}$ ring spectra. We do not discuss whether an $E_{n+1}$ ring spectrum structure on $R$, for some finite $n$, suffices to define a more-or-less strictly coassociative coproduct or Hopf algebra structure on $T H H(R)$.

Proposition 4.3 Let $R$ be an $E_{2}$ ring spectrum. Then the Bökstedt spectral sequence $E_{* *}^{r}(R)$ converging to $H_{*}\left(T H H(R) ; \mathbb{F}_{p}\right)$ is an $A_{*}$-comodule $\mathbb{F}_{p}$-algebra spectral sequence. If $R$ is an $E_{3}$ ring spectrum, then $E_{* *}^{r}(R)$ is a commutative $H_{*}\left(R ; \mathbb{F}_{p}\right)$-algebra spectral sequence in $A_{*}$-comodules. 
Proof To make sense of $T H H(R)$, we have implicitly replaced $R$ with a weakly equivalent associative $S$-algebra. By the discussion above, for $R$ an $E_{2}$ ring spectrum there is a $\mathcal{C}_{1}$-algebra action on the cyclic $S$-module $T H H_{\bullet}(R)$, on the $A_{*}$-comodule $H_{*}\left(T H H(R) ; \mathbb{F}_{p}\right)$ and on the Bökstedt spectral sequence $E_{* *}^{r}(R)$. This $\mathcal{C}_{1}$-action induces the desired $A_{*}$-comodule $\mathbb{F}_{p}$-algebra structures. For example, the spectral sequence pairing is the composite

$$
E_{* *}^{r}(R) \otimes_{\mathbb{F}_{p}} E_{* *}^{r}(R) \rightarrow{ }^{\prime} E_{* *}^{r} \stackrel{\mu}{\longrightarrow} E_{* *}^{r}(R),
$$

with ' $E_{* *}^{r}$ the spectral sequence associated to $T H H(R) \wedge T H H(R)$ and $\mu$ induced by any point in $\mathcal{C}_{1}(2)$. When $R$ is an $E_{3}$ ring spectrum, THH(R) is an $E_{2}$ ring spectrum and homotopy commutative, so the algebra structures are commutative. The unit $S$-algebra map $\eta: R \rightarrow T H H(R)$ is then homotopy central, and supplies the $H_{*}\left(R ; \mathbb{F}_{p}\right)$-algebra structure.

Here is a typical application of this algebra structure.

Corollary 4.4 If $R$ is an $E_{2}$ ring spectrum and $H_{*}\left(R ; \mathbb{F}_{p}\right)$ is a polynomial algebra over $\mathbb{F}_{p}$, then $E_{* *}^{r}(R)$ collapses at the $E^{2}$-term, so $E_{* *}^{2}(R)=E_{* *}^{\infty}(R)$. Furthermore, there are no nontrivial (left or right) $H_{*}\left(R ; \mathbb{F}_{p}\right)$-module extensions.

Proof If $H_{*}\left(R ; \mathbb{F}_{p}\right)=P\left(x_{i}\right)$ is a polynomial algebra, where $i$ ranges through some indexing set $I$, then $E_{* *}^{2}(R)=H H_{*}\left(P\left(x_{i}\right)\right)=P\left(x_{i}\right) \otimes E\left(\sigma x_{i}\right)$ by Proposition 2.4 (and passage to colimits). All of the $\mathbb{F}_{p}$-algebra generators are in filtration $\leq 1$, so all differentials on these classes are zero, since the Bökstedt spectral sequence is a right half plane homological spectral sequence. Thus there are no further differentials in this spectral sequence, and $E_{* *}^{2}(R)=E_{* *}^{\infty}(R)$. Furthermore, the $E^{\infty}$-term is a free $H_{*}\left(R ; \mathbb{F}_{p}\right)$-module, so also $H_{*}\left(T H H(R) ; \mathbb{F}_{p}\right) \cong$ $H_{*}\left(R ; \mathbb{F}_{p}\right) \otimes E\left(\sigma x_{i}\right)$ is a free $H_{*}\left(R ; \mathbb{F}_{p}\right)$-module.

There may, as we shall see in Section 5, be multiplicative extensions between $E_{* *}^{\infty}(R)$ and $H_{*}\left(T H H(R) ; \mathbb{F}_{p}\right)$, as well as $A_{*}$-comodule extensions.

A flatness hypothesis is required for the spectral sequence to carry the coproduct and full Hopf algebra structure. Our Sections 5, 6 and 7 will show many examples of Bökstedt spectral sequences with this structure.

Theorem 4.5 Let $R$ be a commutative $S$-algebra and write $\Lambda=H_{*}\left(R ; \mathbb{F}_{p}\right)$.

(a) If $H_{*}\left(T H H(R) ; \mathbb{F}_{p}\right)$ is flat over $\Lambda$, then there is a coproduct

$$
\psi: H_{*}\left(T H H(R) ; \mathbb{F}_{p}\right) \rightarrow H_{*}\left(T H H(R) ; \mathbb{F}_{p}\right) \otimes_{\Lambda} H_{*}\left(T H H(R) ; \mathbb{F}_{p}\right)
$$


and $H_{*}\left(T H H(R) ; \mathbb{F}_{p}\right)$ is an $A_{*}$-comodule $\Lambda$-Hopf algebra.

(b) If each term $E_{* *}^{r}(R)$ for $r \geq 2$ is flat over $\Lambda$, then there is a coproduct

$$
\psi: E_{* *}^{r}(R) \rightarrow E_{* *}^{r}(R) \otimes_{\Lambda} E_{* *}^{r}(R)
$$

and $E_{* *}^{r}(R)$ is an $A_{*}$-comodule $\Lambda$-Hopf algebra spectral sequence. In particular, the differentials $d^{r}$ respect the coproduct $\psi$.

Proof Write $T=T H H(R)$. There is a Künneth spectral sequence with

$$
E_{* *}^{2}=\operatorname{Tor}_{* *}^{\Lambda}\left(H_{*}\left(T ; \mathbb{F}_{p}\right), H_{*}\left(T ; \mathbb{F}_{p}\right)\right) \Longrightarrow H_{*}\left(T \wedge_{R} T ; \mathbb{F}_{p}\right) .
$$

When $H_{*}\left(T ; \mathbb{F}_{p}\right)$ is flat over $\Lambda$ the higher Tor-groups vanish, the spectral sequence collapses, and the map

$$
\psi: H_{*}\left(T ; \mathbb{F}_{p}\right) \rightarrow H_{*}\left(T \wedge_{R} T ; \mathbb{F}_{p}\right) \cong H_{*}\left(T ; \mathbb{F}_{p}\right) \otimes_{\Lambda} H_{*}\left(T ; \mathbb{F}_{p}\right)
$$

induces the coproduct in part (a).

For part (b) let $d E_{* *}^{r}$ be the spectral sequence associated to the skeleton filtration on $d T H H(R)=R \otimes d S^{1}$, and let ' $E_{* *}^{r}$ be the spectral sequence associated to $T \wedge_{R} T=R \otimes\left(S^{1} \vee S^{1}\right)$, as in the proof of Proposition 4.2. Then there are natural maps of spectral sequences

$$
E_{* *}^{r}(R) \stackrel{\pi}{\longleftarrow} d E_{* *}^{r} \stackrel{\psi^{\prime}}{\longrightarrow} E_{* *}^{r} \stackrel{s h}{\longleftarrow} E_{* *}^{r}(R) \otimes_{\Lambda} E_{* *}^{r}(R) .
$$

Here $\pi: d E_{* *}^{r} \rightarrow E_{* *}^{r}(R)$ is an isomorphism for $r \geq 2$, by the algebraic analogue of Lemma 3.8. The map $\psi^{\prime}: d E_{* *}^{r} \rightarrow{ }^{\prime} E_{* *}^{r}$ is induced by the simplicial pinch map $\psi^{\prime}$ from (3.7). As regards the final (shuffle) map sh, we have

$$
{ }^{\prime} E_{s, *}^{1} \cong \Lambda^{\otimes(s+1)} \otimes_{\Lambda} \Lambda^{\otimes(s+1)} \cong E_{s, *}^{1}(R) \otimes_{\Lambda} E_{s, *}^{1}(R)
$$

by the collapsing Künneth spectral sequence for

$$
H_{*}\left(T H H_{s}(R) \wedge_{R} \mathrm{THH}_{s}(R) ; \mathbb{F}_{p}\right),
$$

and the map $s h$ for $r=1$ is the shuffle equivalence from the bigraded tensor product $\left[E_{* *}^{1}(R) \otimes_{\Lambda} E_{* *}^{1}(R)\right]_{s, *}$. By assumption $E_{* *}^{2}(R)=H H_{*}(\Lambda)$ is flat over $\Lambda$, so by the algebraic Künneth spectral sequence and the Eilenberg-Zilber theorem

$$
s h: E_{* *}^{2}(R) \otimes_{\Lambda} E_{* *}^{2}(R) \cong H_{*}\left(E_{* *}^{1}(R) \otimes_{\Lambda} E_{* *}^{1}(R)\right) \stackrel{\cong}{\longrightarrow} H_{*}\left({ }^{\prime} E_{* *}^{1}\right)={ }^{\prime} E_{* *}^{2}
$$

is an isomorphism. Inductively, suppose that $s h$ is an isomorphism for a fixed $r \geq 2$, and that $E_{* *}^{r+1}(R)$ is flat over $\Lambda$. Then by the algebraic Künneth spectral sequence again

$$
s h: E_{* *}^{r+1}(R) \otimes_{\Lambda} E_{* *}^{r+1}(R) \cong H_{*}\left(E_{* *}^{r}(R) \otimes_{\Lambda} E_{* *}^{r}(R)\right) \stackrel{\cong}{\longrightarrow} H_{*}\left({ }^{\prime} E_{* *}^{r}\right)={ }^{\prime} E_{* *}^{r+1}
$$


is also an isomorphism, as desired. The coproduct $\psi$ on $E_{* *}^{r}(R)$ is then the composite map $(s h)^{-1} \circ \psi^{\prime} \circ \pi^{-1}$, for $r \geq 2$. The conjugation $\chi$ on $E_{* *}^{r}(R)$ is more simply defined, as the composite map $\pi \circ \chi^{\prime} \circ \pi^{-1}$.

By Proposition 2.3, the coproduct (and Hopf algebra structure) on the $E^{2}$-term $E_{* *}^{2}(R)=H H_{*}\left(H_{*}\left(R ; \mathbb{F}_{p}\right)\right)$ that is derived from the $R$-Hopf algebra structure on $T H H(R)$ agrees with the algebraically defined structure on the Hochschild homology $H H_{*}(\Lambda)$ of the commutative algebra $\Lambda=H_{*}\left(R ; \mathbb{F}_{p}\right)$.

Remark 4.7 The same proof shows that if only the initial terms

$$
E_{* *}^{2}(R), \ldots, E_{* *}^{r_{0}}(R)
$$

of the Bökstedt spectral sequence are flat over $\Lambda$, for some integer $r_{0}$, then these are all $A_{*}$-comodule $\Lambda$-Hopf algebras and the corresponding differentials $d^{2}, \ldots, d^{r_{0}}$ all respect that structure.

There are natural examples that show that the flatness hypothesis is not always realistic. For example, Ausoni $\mathrm{Au}$ ] has studied the case of $R=k u$ at an odd prime $p$, where $H_{*}\left(k u ; \mathbb{F}_{p}\right)=H_{*}\left(\ell ; \mathbb{F}_{p}\right) \otimes P_{p-1}(x)$ for a class $x$ in degree 2 , and already the $E^{2}$-term $E_{* *}^{2}(k u)=H H_{*}\left(H_{*}\left(k u ; \mathbb{F}_{p}\right)\right)$ is not flat over $H_{*}\left(k u ; \mathbb{F}_{p}\right)$. We shall also see in Proposition 7.13 (c) that for $R=j$, the connective, real image-of- $J$ spectrum at $p=2$, the $E^{2}$-term $E_{* *}^{2}(j)=H H_{*}\left(H_{*}\left(j ; \mathbb{F}_{2}\right)\right)$ is not flat over $H_{*}\left(j ; \mathbb{F}_{2}\right)$.

We say that the graded $k$-algebra $\Lambda$ is connected when it is trivial in negative degrees and the unit map $\eta: k \rightarrow \Lambda$ is an isomorphism in degree 0 . We write $\nu: H_{*}\left(X ; \mathbb{F}_{p}\right) \rightarrow A_{*} \otimes H_{*}\left(X ; \mathbb{F}_{p}\right)$ for the $A_{*}$-comodule coaction map, and say that a class $x \in H_{*}\left(X ; \mathbb{F}_{p}\right)$ is $A_{*}$-comodule primitive if $\nu(x)=1 \otimes x$.

Proposition 4.8 Let $R$ be a commutative $S$-algebra with $\Lambda=H_{*}\left(R ; \mathbb{F}_{p}\right)$ connected and such that $H H_{*}(\Lambda)$ is flat over $\Lambda$. Then the $E^{2}$-term of the first quadrant Bökstedt spectral sequence

$$
E_{* *}^{2}(R)=H H_{*}(\Lambda)
$$

is an $A_{*}$-comodule $\Lambda$-Hopf algebra, and a shortest non-zero differential $d_{s, t}^{r}$ in lowest total degree $s+t$, if one exists, must map from an algebra indecomposable to a coalgebra primitive and $A_{*}$-comodule primitive, in $H H_{*}(\Lambda)$.

Proof If $d^{2}, \ldots, d^{r-1}$ are all zero, then $E_{* *}^{2}(R)=E_{* *}^{r}(R)$ is still an $A_{*^{-}}$ comodule $\Lambda$-Hopf algebra. If $d^{r}(x y) \neq 0$, where $x y$ is decomposable (a product of classes of positive degree), then the Leibniz formula

$$
d^{r}(x y)=d^{r}(x) y \pm x d^{r}(y)
$$


implies that $d^{r}(x) \neq 0$ or $d^{r}(y) \neq 0$, so $x y$ cannot be in the lowest possible total degree for the source of a differential. Dually, if $d^{r}(z)$ is not coalgebra primitive, with $\psi(z)=z \otimes 1+1 \otimes z+\sum_{i} z_{i}^{\prime} \otimes z_{i}^{\prime \prime}$, then the co-Leibniz formula

$$
\psi \circ d^{r}=\left(d^{r} \otimes 1 \pm 1 \otimes d^{r}\right) \psi
$$

(tensor products over $\Lambda$ ) implies that some term $d^{r}\left(z_{i}^{\prime}\right) \neq 0$ or $d^{r}\left(z_{i}^{\prime \prime}\right) \neq 0$, so $z$ cannot be in the lowest possible total degree. Finally, if $d^{r}(z)$ is not $A_{*}$-comodule primitive, with $\nu(z)=1 \otimes z+\sum_{i} a_{i} \otimes z_{i}$, then the co-linearity condition

$$
\nu \circ d^{r}=\left(1 \otimes d^{r}\right) \nu
$$

implies that some term $d^{r}\left(z_{i}\right) \neq 0$, so $z$ cannot be in the lowest possible total degree.

The last two arguments are perhaps easier to visualize in the $\mathbb{F}_{p}$-vector space dual spectral sequence. The $\mathbb{F}_{p}$-dual of the coproduct $\psi$ maps from the cotensor product

$$
H H_{*}(\Lambda)^{*} \square_{\Lambda^{*}} H H_{*}(\Lambda)^{*} \subset H H_{*}(\Lambda)^{*} \otimes_{\mathbb{F}_{p}} H H_{*}(\Lambda)^{*}
$$

to $H H_{*}(\Lambda)^{*}$.

Proposition 4.9 Let $R$ be a commutative $S$-algebra. For each element $x \in$ $H_{t}\left(R ; \mathbb{F}_{p}\right)$ the image $\sigma x \in H_{t+1}\left(T H H(R) ; \mathbb{F}_{p}\right)$ is the coalgebra primitive

$$
\psi(\sigma x)=\sigma x \otimes 1+1 \otimes \sigma x
$$

that is represented in $E_{* *}^{\infty}(R)$ by the class $\sigma x=[1 \otimes x] \in E_{1, t}^{2}(R)=H H_{1}(\Lambda)_{t}$.

Proof Note that the coproduct on $\operatorname{THH}(R)$ is compatible under $\omega: R \wedge S_{+}^{1} \rightarrow$ $T H H(R)$ with the pinch map $R \wedge d S_{+}^{1} \rightarrow R \wedge\left(S^{1} \vee S^{1}\right)_{+}$, and thus under $\sigma: \Sigma R \rightarrow T H H(R)$ with the pinch map $\Sigma R \rightarrow \Sigma R \vee \Sigma R$. The claims then follow by inspection of the definitions in Section 3 .

See also MS93, 3.2] for the last claim above.

\section{$5 \quad$ Differentials and algebra extensions}

We now apply the Bökstedt spectral sequence (4.1) to compute the mod $p$ homology of $T H H(R)$ for the Brown-Peterson and Johnson-Wilson $S$-algebras $R=B P\langle m-1\rangle$ for $0 \leq m \leq \infty$. In each case we can replace $R$ by its $p$ localization $R_{(p)}$ or $p$-completion $R_{p}$ without changing the $\bmod p$ homology 
of $\operatorname{THH}(R)$, so we will sometimes do so without further comment. The cases $R=H \mathbb{F}_{p}$ and $R=H \mathbb{Z}$ were first treated by Bökstedt [Bo2], and the case $R=\ell_{p} \subset k u_{p}$ (the Adams summand of $p$-complete connective $K$-theory) is due to McClure and Staffeldt [MS93, 4.2].

\subsection{The dual Steenrod algebra}

Let $A=H^{*}\left(H \mathbb{F}_{p} ; \mathbb{F}_{p}\right)$ be the Steenrod algebra, with generators $S q^{i}$ for $p=2$ and $\beta$ and $P^{i}$ for $p$ odd. We recall the structure of its dual $A_{*}=H_{*}\left(H \mathbb{F}_{p} ; \mathbb{F}_{p}\right)$ from [Mi60, Thm. 2]. When $p=2$ we have

$$
A_{*}=P\left(\xi_{k} \mid k \geq 1\right)=P\left(\bar{\xi}_{k} \mid k \geq 1\right)
$$

where $\xi_{k}$ has degree $2^{k}-1$ and $\bar{\xi}_{k}=\chi\left(\xi_{k}\right)$ is the conjugate class. Most of the time it will be more convenient for us to use the conjugate classes. The coproduct is given by

$$
\psi\left(\bar{\xi}_{k}\right)=\sum_{i+j=k} \bar{\xi}_{i} \otimes \bar{\xi}_{j}^{2^{i}}
$$

where as usual we read $\bar{\xi}_{0}$ to mean 1 . When $p$ is odd we have

$$
A_{*}=P\left(\bar{\xi}_{k} \mid k \geq 1\right) \otimes E\left(\bar{\tau}_{k} \mid k \geq 0\right)
$$

with $\bar{\xi}_{k}=\chi\left(\xi_{k}\right)$ in degree $2\left(p^{k}-1\right)$ and $\bar{\tau}_{k}=\chi\left(\tau_{k}\right)$ in degree $2 p^{k}-1$. The coproduct is given by

$$
\psi\left(\bar{\xi}_{k}\right)=\sum_{i+j=k} \bar{\xi}_{i} \otimes \bar{\xi}_{j}^{p^{i}} \quad \text { and } \quad \psi\left(\bar{\tau}_{k}\right)=1 \otimes \bar{\tau}_{k}+\sum_{i+j=k} \bar{\tau}_{i} \otimes \bar{\xi}_{j}^{p^{i}} .
$$

The mod $p$ homology Bockstein satisfies $\beta\left(\bar{\tau}_{k}\right)=\bar{\xi}_{k}$.

Any commutative $S$-algebra $R$ has a canonical structure as an $E_{\infty}$ ring spectrum [EKMM97, II.3.4]. In particular, its $\bmod p$ homology $H_{*}\left(R ; \mathbb{F}_{p}\right)$ admits natural Dyer-Lashof operations

$$
Q^{k}: H_{*}\left(R ; \mathbb{F}_{2}\right) \rightarrow H_{*+k}\left(R ; \mathbb{F}_{2}\right)
$$

for $p=2$ and

$$
Q^{k}: H_{*}\left(R ; \mathbb{F}_{p}\right) \rightarrow H_{*+2 k(p-1)}\left(R ; \mathbb{F}_{p}\right)
$$

for $p$ odd. Their formal properties are summarized in [BMMS86, III.1.1], and include Cartan formulas, Adem relations and Nishida relations. For $p=2$, $Q^{k}(x)=0$ when $k<|x|$ and $Q^{k}(x)=x^{2}$ when $k=|x|$. For $p$ odd, $Q^{k}(x)=0$ when $k<2|x|$ and $Q^{k}(x)=x^{p}$ when $k=2|x|$. In the special case of $R=H \mathbb{F}_{p}$, the Dyer-Lashof operations in $A_{*}=H_{*}\left(H \mathbb{F}_{p} ; \mathbb{F}_{p}\right)$ satisfy

$$
Q^{p^{k}}\left(\bar{\xi}_{k}\right)=\bar{\xi}_{k+1}
$$


for all primes $p$, and

$$
Q^{p^{k}}\left(\bar{\tau}_{k}\right)=\bar{\tau}_{k+1}
$$

for $p$ odd. These formulas were first obtained by Leif Kristensen (unpublished), and appeared in print in BMMS86, III.2.2 and III.2.3].

When $R$ is just an $E_{n}$ ring spectrum, for $1 \leq n<\infty$, only some of these operations are naturally defined. For $p=2$ the operations $Q^{k}(x)$ are defined for $k \leq|x|+n-1$, and for $p$ odd they are defined for $2 k \leq|x|+n-1$. In the case of equality, the "top" operation $Q^{k}(x)$ (denoted $\xi_{n-1}(x)$ in op. cit.) is not additive, and special care is called for. We refer to BMMS86. III.3.1-3] for detailed information about the formal properties that are satisfied in the homology of an $E_{n}$ ring spectrum.

\subsection{The Johnson-Wilson spectra $B P\langle m-1\rangle$}

For any prime $p$ let $B P=B P\langle\infty\rangle$ be the Brown-Peterson spectrum and let $B P\langle m-1\rangle$ for $0 \leq m<\infty$ be the spectrum introduced by Johnson and Wilson in JW73, with mod $p$ cohomology

$$
H^{*}\left(B P ; \mathbb{F}_{p}\right) \cong A / / E=A \otimes_{E} \mathbb{F}_{p}
$$

and

$$
H^{*}\left(B P\langle m-1\rangle ; \mathbb{F}_{p}\right) \cong A / / E_{m-1}=A \otimes_{E_{m-1}} \mathbb{F}_{p} .
$$

Here $E \subset A$ is the exterior subalgebra generated by the Milnor primitives $Q_{k}$ for $k \geq 0$, while $E_{m-1}$ is the exterior subalgebra generated by $Q_{0}, \ldots, Q_{m-1}$. These elements are inductively defined by $Q_{0}=S q^{1}$ and $Q_{k}=\left[S q^{2^{k}}, Q_{k-1}\right]$ for $p=2$, and by $Q_{0}=\beta$ and $Q_{k+1}=\left[P^{p^{k}}, Q_{k}\right]$ for $p$ odd, see [Mi60, $\left.\S 1\right]$. We note the special cases $B P\langle-1\rangle=H \mathbb{F}_{p}, B P\langle 0\rangle=H \mathbb{Z}_{(p)}$ and $B P\langle 1\rangle=\ell \subset k u_{(p)}$. For $p=2$ we have $\ell=k u_{(2)}$.

The Brown-Peterson and Johnson-Wilson spectra have homotopy groups

$$
\pi_{*} B P=\mathbb{Z}_{(p)}\left[v_{k} \mid k \geq 1\right]
$$

for $m=\infty$ and

$$
\pi_{*} B P\langle m-1\rangle=\mathbb{Z}_{(p)}\left[v_{1}, \ldots, v_{m-1}\right]=\pi_{*} B P /\left(v_{k} \mid k \geq m\right)
$$

for $0 \leq m<\infty$, with $v_{0}=p$. The class $v_{k}$ is detected in the Adams spectral sequence $E_{2}^{* *}=\operatorname{Ext}_{A_{*}}^{* *}\left(\mathbb{F}_{p}, H_{*}\left(B P ; \mathbb{F}_{p}\right)\right)$ for $\pi_{*} B P$ by the normalized cobar cocycle

$$
\sum_{i+j=k+1}\left[\bar{\xi}_{i}\right] \bar{\xi}_{j}^{2^{i}}
$$


for $p=2$ (the term for $i=0$ is zero) and

$$
-\sum_{i+j=k}\left[\bar{\tau}_{i}\right] \bar{\xi}_{j}^{p^{i}}
$$

for $p$ odd [Ra04, p. 63]. Under the change-of-rings isomorphism to $E_{2}^{* *} \cong$ $\operatorname{Ext}_{E_{*}}^{* *}\left(\mathbb{F}_{p}, \mathbb{F}_{p}\right)$, where $E_{*}=E\left(\bar{\xi}_{k} \mid k \geq 1\right)$ for $p=2$ and $E_{*}=E\left(\bar{\tau}_{k} \mid k \geq 0\right)$ for $p$ odd, these cobar cocycles correspond to $\left[\bar{\xi}_{k+1}\right]$ and $-\left[\bar{\tau}_{k}\right]$, respectively. Modulo decomposables, we have $\bar{\xi}_{k+1} \equiv \xi_{k+1}$ for $p=2$ and $-\bar{\tau}_{k} \equiv \tau_{k}$ for $p$ odd.

In each case $0 \leq m \leq \infty$, the spectrum $B P\langle m-1\rangle$ admits the structure of an associative $S$-algebra. More precisely, there is a tower of associative $M U$ algebras

$$
B P \rightarrow \cdots \rightarrow B P\langle m\rangle \rightarrow B P\langle m-1\rangle \rightarrow \cdots \rightarrow H \mathbb{F}_{p},
$$

which by omission of structure is also a tower of $S$-algebras. See [BJ02, 3.5]. It is well known that $H \mathbb{F}_{p}$ and $H \mathbb{Z}_{(p)}$ admit unique structures as commutative $S$-algebras, and that the $p$-complete Adams summand $\ell_{p} \subset k u_{p}$ admits at least one such structure [MS93, §9]. In fact each of $\ell, k u, \ell_{p}$ and $k u_{p}$ admits a unique commutative $S$-algebra structure $\mathrm{BaRi}$.

It remains a well-known open problem whether $B P$ admits a commutative $S$ algebra structure. Basterra and Mandell (unpublished) have shown that $B P$ is at least an $E_{4}$ ring spectrum. We shall make use of the existence of an $E_{3}$ ring spectrum structure on $B P$ for the calculations in this section.

For $p=2$ and $2<m<\infty$, Strickland [Str99, 6.5] has shown that $B P\langle m-1\rangle$ is not a homotopy commutative $M U$-ring spectrum with the most common (e.g. Araki or Hazewinkel) choices of maps $M U \rightarrow B P\langle m-1\rangle$, but he also shows that there is a less familiar replacement $B P\langle m-1\rangle^{\prime}$ that may serve as a substitute [Str99, 2.10]. We do not know for which $p$ and $m$ the spectrum $B P\langle m-1\rangle$ admits an $E_{2}$ - or $E_{3}$ ring spectrum structure.

Proposition 5.3 For each $p$ and $0 \leq m \leq \infty$ the unique $S$-algebra map $B P\langle m-1\rangle \rightarrow H \mathbb{F}_{p}$ induces the identifications

$$
H_{*}\left(B P\langle m-1\rangle ; \mathbb{F}_{2}\right)=P\left(\bar{\xi}_{1}^{2}, \ldots, \bar{\xi}_{m}^{2}, \bar{\xi}_{k} \mid k \geq m+1\right)
$$

when $p=2$, and

$$
H_{*}\left(B P\langle m-1\rangle ; \mathbb{F}_{p}\right)=P\left(\bar{\xi}_{k} \mid k \geq 1\right) \otimes E\left(\bar{\tau}_{k} \mid k \geq m\right)
$$

when $p$ is odd, as $\mathbb{F}_{p}$-subalgebras of $A_{*}$. 
Proof See Wi75, 1.7] and dualize. In the case $m=\infty$, the notation is meant to imply that there are no classes $\bar{\xi}_{k}$ with $k \geq m+1$, and no classes $\bar{\tau}_{k}$ with $k \geq m$.

In particular,

$$
\begin{aligned}
H_{*}\left(H \mathbb{Z} ; \mathbb{F}_{2}\right) & =P\left(\bar{\xi}_{1}^{2}, \bar{\xi}_{k} \mid k \geq 2\right) \\
H_{*}\left(k u ; \mathbb{F}_{2}\right) & =P\left(\bar{\xi}_{1}^{2}, \bar{\xi}_{2}^{2}, \bar{\xi}_{k} \mid k \geq 3\right) \\
H_{*}\left(B P ; \mathbb{F}_{2}\right) & =P\left(\bar{\xi}_{k}^{2} \mid k \geq 1\right)
\end{aligned}
$$

for $p=2$, and

$$
\begin{aligned}
H_{*}\left(H \mathbb{Z} ; \mathbb{F}_{p}\right) & =P\left(\bar{\xi}_{k} \mid k \geq 1\right) \otimes E\left(\bar{\tau}_{k} \mid k \geq 1\right) \\
H_{*}\left(\ell ; \mathbb{F}_{p}\right) & =P\left(\bar{\xi}_{k} \mid k \geq 1\right) \otimes E\left(\bar{\tau}_{k} \mid k \geq 2\right) \\
H_{*}\left(B P ; \mathbb{F}_{p}\right) & =P\left(\bar{\xi}_{k} \mid k \geq 1\right)
\end{aligned}
$$

for $p$ odd.

The $E^{2}$-term of the Bökstedt spectral sequence for $B P\langle m-1\rangle$ can now be computed from Proposition 2.4 It is

$$
E_{* *}^{2}(B P\langle m-1\rangle)=H_{*}\left(B P\langle m-1\rangle ; \mathbb{F}_{2}\right) \otimes E\left(\sigma \bar{\xi}_{1}^{2}, \ldots, \sigma \bar{\xi}_{m}^{2}, \sigma \bar{\xi}_{k} \mid k \geq m+1\right)
$$

when $p=2$, and

$$
E_{* *}^{2}(B P\langle m-1\rangle)=H_{*}\left(B P\langle m-1\rangle ; \mathbb{F}_{p}\right) \otimes E\left(\sigma \bar{\xi}_{k} \mid k \geq 1\right) \otimes \Gamma\left(\sigma \bar{\tau}_{k} \mid k \geq m\right)
$$

when $p$ is odd. Note that in all cases for $p=2$, as well as the case $m=\infty$ for $p$ odd, the $E^{2}$-term is generated as an $\mathbb{F}_{p}$-algebra by classes in filtration $\leq 1$.

\subsection{Odd-primary differentials}

In the remaining cases, with $p$ odd and $0 \leq m<\infty$, there are non-trivial $d^{p-1}$ differentials in the Bökstedt spectral sequence. These can all be determined by naturality with respect to the map of $S$-algebras $B P\langle m-1\rangle \rightarrow H \mathbb{F}_{p}$, since the map of $E^{2}$-terms

$$
E_{* *}^{2}(B P\langle m-1\rangle) \rightarrow E_{* *}^{2}\left(H \mathbb{F}_{p}\right)=A_{*} \otimes E\left(\sigma \bar{\xi}_{k} \mid k \geq 1\right) \otimes \Gamma\left(\sigma \bar{\tau}_{k} \mid k \geq 0\right)
$$

is injective. Now $H \mathbb{F}_{p}$ is a connective, commutative $S$-algebra, and the $E^{2}$ term is free, hence flat, over $A_{*}=H_{*}\left(H \mathbb{F}_{p} ; \mathbb{F}_{p}\right)$, so we are in the situation of Proposition 4.8. The $A_{*}$-algebra generators (indecomposables) of the $E^{2}$-term are in filtrations $p^{i}$ for $i \geq 0$, by formula (2.5), and the $A_{*}$-coalgebra primitives are all in filtration 1 , by Proposition 2.4 so the shortest non-zero differential 
in lowest total degree, if any, must go from some filtration $p^{i}$ for $i \geq 1$ to filtration 1 .

Indeed, Bökstedt [Bo2, 1.3] found that in the case $R=H \mathbb{F}_{p}$ there are nontrivial $d^{p-1}$-differentials in his spectral sequence computing $H_{*}\left(T H H(R) ; \mathbb{F}_{p}\right)$. In our notation, they are given by the formula

$$
d^{p-1}\left(\gamma_{j}\left(\sigma \bar{\tau}_{k}\right)\right)=\sigma \bar{\xi}_{k+1} \cdot \gamma_{j-p}\left(\sigma \bar{\tau}_{k}\right)
$$

for $j \geq p$, up to a unit in $\mathbb{F}_{p}$. This way of writing Bökstedt's formula first appears in [MS93, p. 21]. A proof of a more general result that implies (5.5) was published by Hunter [Hu96, Thm. 1], as recalled below.

Proposition 5.6 Let $R$ be a commutative $S$-algebra, and let $x \in H_{2 i-1}\left(R ; \mathbb{F}_{p}\right)$ for some $i \geq 1$. Then in the Bökstedt spectral sequence $E_{* *}^{r}(R)$ the differentials $d^{r}$ all vanish for $2 \leq r \leq p-2$, and there is a differential

$$
d^{p-1}\left(\gamma_{p}(\sigma x)\right)=\sigma\left(\beta Q^{i}(x)\right)
$$

up to a unit in $\mathbb{F}_{p}$.

A short proof of Bökstedt's formula in particular cases was later found by Ausoni [Au, 4.3]. More recently, the first author has given a construction of $\mathrm{THH}$ of any $A_{\infty}$ ring spectrum as a kind of geometric realization using cyclohedra [Sta97], similar to Stasheff's bar construction of an $A_{\infty} H$ space in Sta63. This construction exhibits the $d^{p-1}$-differential on $\gamma_{p}(\sigma x)$ as being represented by $\sigma(\langle x, \ldots, x\rangle)$ in the Bökstedt spectral sequence, where $\langle x, \ldots, x\rangle \in H_{2 p i-2}\left(R ; \mathbb{F}_{p}\right)$ denotes the $p$-fold Massey product. In this formulation only the strict associativity of $R$ is needed.

The proposition above applies with $R=H \mathbb{F}_{p}, x=\bar{\tau}_{k}, i=p^{k}$ and $\beta Q^{i}(x)=$ $\beta \bar{\tau}_{k+1}=\bar{\xi}_{k+1}$, to establish Bökstedt's formula (5.5) in the special case of $j=p$. The general case follows from this by induction on $j \geq p$ and the coalgebra structure on the Bökstedt spectral sequence. In more detail, a comparison of $\psi d^{p-1}\left(\gamma_{j}\left(\sigma \bar{\tau}_{k}\right)\right)=\left(d^{p-1} \otimes 1+1 \otimes d^{p-1}\right)\left(\psi \gamma_{j}\left(\sigma \bar{\tau}_{k}\right)\right)$ and $\psi\left(\sigma \bar{\xi}_{k+1} \cdot \gamma_{j-p}\left(\sigma \bar{\tau}_{k}\right)\right)$ shows that the difference $d^{p-1}\left(\gamma_{j}\left(\sigma \bar{\tau}_{k}\right)\right)-\sigma \bar{\xi}_{k+1} \cdot \gamma_{j-p}\left(\sigma \bar{\tau}_{k}\right)$ must be a coalgebra primitive, and there are none such other than zero in its bidegree when $j>p$.

By naturality, the formula (5.5) for $d^{p-1}$ holds also in the Bökstedt spectral sequence for $B P\langle m-1\rangle$ at odd primes $p$, whether this $S$-algebra is commutative or not. We view its $E^{2}=E^{p-1}$-term as the tensor product of the complexes $E\left(\sigma \bar{\xi}_{k+1}\right) \otimes \Gamma\left(\sigma \bar{\tau}_{k}\right)$ for $k \geq m$ and the remaining terms $H_{*}\left(B P\langle m-1\rangle ; \mathbb{F}_{p}\right) \otimes$ 
$E\left(\sigma \bar{\xi}_{1}, \ldots, \sigma \bar{\xi}_{m}\right)$. Applying the Künneth formula, we compute its homology to be

$$
E_{* *}^{p}(B P\langle m-1\rangle)=H_{*}\left(B P\langle m-1\rangle ; \mathbb{F}_{p}\right) \otimes E\left(\sigma \bar{\xi}_{1}, \ldots, \sigma \bar{\xi}_{m}\right) \otimes P_{p}\left(\sigma \bar{\tau}_{k} \mid k \geq m\right) .
$$

At this point we note that also for $0 \leq m<\infty$ and $p$ odd, the $E^{p}$-term of the Bökstedt spectral sequence is generated as an $\mathbb{F}_{p}$-algebra by classes in filtration $\leq 1$.

To proceed, we shall need to assume that the Bökstedt spectral sequence is an algebra spectral sequence. We know that the hypothesis of the following proposition is satisfied in the commutative cases $0 \leq m \leq 2$ and in the $E_{4}$ ring spectrum case $m=\infty$.

Proposition 5.7 Let $p$ and $0 \leq m \leq \infty$ be such that $B P\langle m-1\rangle$ admits the structure of an $E_{2}$ ring spectrum. Then its Bökstedt spectral sequence collapses at the $E^{p}=E^{\infty}$-term, which equals

$$
E_{* *}^{\infty}(B P\langle m-1\rangle)=H_{*}\left(B P\langle m-1\rangle ; \mathbb{F}_{2}\right) \otimes E\left(\sigma \bar{\xi}_{1}^{2}, \ldots, \sigma \bar{\xi}_{m}^{2}, \sigma \bar{\xi}_{k} \mid k \geq m+1\right)
$$

when $p=2$, and

$$
E_{* *}^{\infty}(B P\langle m-1\rangle)=H_{*}\left(B P\langle m-1\rangle ; \mathbb{F}_{p}\right) \otimes E\left(\sigma \bar{\xi}_{1}, \ldots, \sigma \bar{\xi}_{m}\right) \otimes P_{p}\left(\sigma \bar{\tau}_{k} \mid k \geq m\right)
$$

when $p$ is odd. In each case the $E^{\infty}$-term exhibits $H_{*}\left(T H H(B P\langle m-1\rangle) ; \mathbb{F}_{p}\right)$ as a free $H_{*}\left(B P\langle m-1\rangle ; \mathbb{F}_{p}\right)$-module.

Proof By Proposition 4.3 we know that the Bökstedt spectral sequence is an $\mathbb{F}_{p}$-algebra spectral sequence, and by the calculations above we know that its $E^{p}$-term is generated as an $\mathbb{F}_{p}$-algebra by classes in filtrations $\leq 1$, which must be infinite cycles since the spectral sequence is concentrated in the first quadrant. Thus there is no room for any further differentials and $E^{p}=E^{\infty}$. In each case the $E^{\infty}$-term is free as an $H_{*}\left(B P\langle m-1\rangle ; \mathbb{F}_{p}\right)$-module, so there is no room for additive (module-)extensions, either.

\subsection{Algebra extensions}

To determine $H_{*}\left(T H H(B P\langle m-1\rangle) ; \mathbb{F}_{p}\right)$ as an $H_{*}\left(B P\langle m-1\rangle ; \mathbb{F}_{p}\right)$-algebra, we need to resolve the possible multiplicative extensions. For this we will use the first nontrivial Dyer-Lashof operation after the Pontryagin power, the existence of which presupposes that $T H H(B P\langle m-1\rangle)$ is an $E_{2}$ ring spectrum. We shall therefore assume that $p$ and $m$ are such that $B P\langle m-1\rangle$ is an $E_{3}$ ring spectrum. 
For a commutative $S$-algebra $R$, the map $\sigma: \Sigma R \rightarrow T H H(R)$ relates the Dyer-Lashof operations on $R$ to those of $\operatorname{THH}(R)$ by the following formula of Bökstedt [Bo2, 2.9]:

$$
Q^{k}(\sigma x)=\sigma Q^{k}(x) .
$$

See also MS93 p. 22]. There appears to be no published proof of this key relation, so we offer the following slightly more general result.

Recall from Definition [2.6 the $S^{1}$-action map $\alpha: T H H(R) \wedge S_{+}^{1} \rightarrow T H H(R)$, inducing the homomorphism

$$
\begin{aligned}
\alpha: H_{*}\left(T H H(R) ; \mathbb{F}_{p}\right) \otimes H_{*}\left(S_{+}^{1} ; \mathbb{F}_{p}\right) \cong \\
H_{*}\left(T H H(R) \wedge S_{+}^{1} ; \mathbb{F}_{p}\right) \rightarrow H_{*}\left(T H H(R) ; \mathbb{F}_{p}\right)
\end{aligned}
$$

in homology. Let $s_{1} \in H_{1}\left(S_{+}^{1} ; \mathbb{F}_{p}\right)$ be the canonical generator. Also recall that any commutative $S$-algebra $R$ is an $E_{\infty}$ ring spectrum, so that the following proposition applies to such an $R$ with $n=\infty$, and thus for all integers $k$.

Proposition 5.9 Let $R$ be an $E_{n+1}$ ring spectrum, so that $T H H(R)$ is an $E_{n}$ ring spectrum. Then we have

$$
Q^{k}\left(\alpha\left(x \otimes s_{1}\right)\right)=\alpha\left(Q^{k}(x) \otimes s_{1}\right)
$$

for all classes $x \in H_{*}\left(T H H(R) ; \mathbb{F}_{p}\right)$ and integers $k$ such that the operation $Q^{k}(x)$ is naturally defined, i.e., for $k \leq|x|+n-1$ when $p=2$ and for $2 k \leq|x|+n-1$ when $p$ is odd. In particular, we have

$$
Q^{k}(\sigma x)=\sigma Q^{k}(x)
$$

for all classes $x \in H_{*}\left(R ; \mathbb{F}_{p}\right)$ and the same integers $k$.

Proof By [FV, 3.4], or the definition $T H H(R) \cong R \otimes S^{1}$ in the strictly commutative case, the circle acts on $T H H(R)$ by $\mathcal{C}_{n}$-algebra maps. Thus the right adjoint map

$$
\widetilde{\alpha}: T H H(R) \rightarrow F\left(S_{+}^{1}, T H H(R)\right)
$$

is a map of $\mathcal{C}_{n}$-algebras, where the product structure on the right is given by pointwise multiplication, using the strictly cocommutative diagonal map $S_{+}^{1} \rightarrow S_{+}^{1} \wedge S_{+}^{1}$.

Let $D S_{+}^{1}=F\left(S_{+}^{1}, S\right)$ be the functional dual of $S_{+}^{1}$, also with the pointwise multiplication. It has $\bmod p$ homology $H_{*}\left(D S_{+}^{1} ; \mathbb{F}_{p}\right) \cong H^{-*}\left(S_{+}^{1} ; \mathbb{F}_{p}\right)=E\left(\iota_{1}\right)$ for a canonical class $\iota_{1} \in H^{1}\left(S_{+}^{1} ; \mathbb{F}_{p}\right)$ dual to the class $s_{1} \in H_{1}\left(S_{+}^{1} ; \mathbb{F}_{p}\right)$. The Dyer-Lashof operations $Q^{k}$ on $H_{*}\left(D S_{+}^{1} ; \mathbb{F}_{p}\right)$ correspond [BMMS86, III.1.2 and 
VIII.3] to the Steenrod operations $P^{-k}$ on $H^{-*}\left(S_{+}^{1} ; \mathbb{F}_{p}\right)$, hence are trivial for $k \neq 0$. The same conclusions hold for $p=2$, with somewhat different notation.

There is a canonical map of $\mathcal{C}_{n}$-algebras

$$
\nu: T H H(R) \wedge D S_{+}^{1} \rightarrow F\left(S_{+}^{1}, T H H(R)\right),
$$

given by the composition of functions

$$
F(S, T H H(R)) \wedge F\left(S_{+}^{1}, S\right) \rightarrow F\left(S_{+}^{1}, T H H(R)\right) .
$$

Compare [LMS86, III.1]. The map $\nu$ is an equivalence since $S_{+}^{1}$ is a finite CW complex (i.e., by Spanier-Whitehead duality). Hence there are homomorphisms

$$
\begin{aligned}
H_{*}\left(T H H(R) ; \mathbb{F}_{p}\right) \stackrel{\tilde{\alpha}}{\longrightarrow} H_{*}\left(F\left(S_{+}^{1}, T H H(R)\right) ; \mathbb{F}_{p}\right) \\
\cong \uparrow \\
H_{*}\left(T H H(R) ; \mathbb{F}_{p}\right) \otimes H_{*}\left(D S_{+}^{1} ; \mathbb{F}_{p}\right)
\end{aligned}
$$

that take $x \in H_{*}\left(T H H(R) ; \mathbb{F}_{p}\right)$ to

$$
\nu^{-1} \widetilde{\alpha}(x)=x \otimes 1+\alpha\left(x \otimes s_{1}\right) \otimes \iota_{1} .
$$

Since $\widetilde{\alpha}$ and $\nu$ are maps of $\mathcal{C}_{n}$-algebras, we have

$$
Q^{k}\left(\nu^{-1} \widetilde{\alpha}(x)\right)=\nu^{-1} \widetilde{\alpha}\left(Q^{k}(x)\right)
$$

when $k$ is such that $Q^{k}$ is naturally defined on $x \in H_{*}\left(T H H(R) ; \mathbb{F}_{p}\right)$. The external Cartan formula for Dyer-Lashof operations [BMMS86, III.1.1(6)] then gives us

$$
Q^{k}\left(x \otimes 1+\alpha\left(x \otimes s_{1}\right) \otimes \iota_{1}\right)=Q^{k}(x) \otimes 1+Q^{k}\left(\alpha\left(x \otimes s_{1}\right)\right) \otimes \iota_{1}
$$

since $Q^{i}(1)=0$ and $Q^{i}\left(\iota_{1}\right)=0$ for $i \neq 0$. Stringing these formulas together yields

$$
Q^{k}(x) \otimes 1+Q^{k}\left(\alpha\left(x \otimes s_{1}\right)\right) \otimes \iota_{1}=Q^{k}(x) \otimes 1+\alpha\left(Q^{k}(x) \otimes s_{1}\right) \otimes \iota_{1},
$$

and we can read off $Q^{k}\left(\alpha\left(x \otimes s_{1}\right)\right)=\alpha\left(Q^{k}(x) \otimes s_{1}\right)$, as desired.

Specializing to classes $\eta(x) \in H_{*}\left(T H H(R) ; \mathbb{F}_{p}\right)$ that are images under the $E_{n}$ ring spectrum map $\eta: R \rightarrow T H H(R)$ of elements $x \in H_{*}\left(R ; \mathbb{F}_{p}\right)$, we have $\sigma x=\alpha\left(\eta(x) \otimes s_{1}\right)$ and $\sigma Q^{k}(x)=\alpha\left(\eta\left(Q^{k}(x)\right) \otimes s_{1}\right)=\alpha\left(Q^{k}(\eta(x)) \otimes s_{1}\right)$. Thus we obtain Bökstedt's formula $Q^{k}(\sigma x)=\sigma Q^{k}(x)$, valid for the same integers $k$ as above.

The same ideas can be used to prove that $\sigma: H_{*}\left(R ; \mathbb{F}_{p}\right) \rightarrow H_{*+1}\left(T H H(R) ; \mathbb{F}_{p}\right)$ is a graded derivation, when $R$ is sufficiently commutative for this to make sense. 
Proposition 5.10 Let $R$ be an $E_{2}$ ring spectrum, so that $T H H(R)$ is an $S$-algebra. Then we have

$$
\alpha\left(x y \otimes s_{1}\right)=x \cdot \alpha\left(y \otimes s_{1}\right)+(-1)^{|y|} \alpha\left(x \otimes s_{1}\right) \cdot y
$$

for $x, y \in H_{*}\left(T H H(R) ; \mathbb{F}_{p}\right)$. In particular, we have the Leibniz rule

$$
\sigma(x \cdot y)=x \cdot \sigma(y)+(-1)^{|y|} \sigma(x) \cdot y
$$

for $x, y \in H_{*}\left(R ; \mathbb{F}_{p}\right)$.

Proof We keep the notation of the proof of Proposition [5.9. Since $\tilde{\alpha}$ and $\nu$ are maps of $\mathcal{C}_{1}$-algebras,

$$
\nu^{-1} \tilde{\alpha}: H_{*}\left(T H H(R) ; \mathbb{F}_{p}\right) \rightarrow H_{*}\left(T H H(R) ; \mathbb{F}_{p}\right) \otimes E\left(\iota_{1}\right)
$$

is an $\mathbb{F}_{p}$-algebra homomorphism. Thus

$$
x y \otimes 1+\alpha\left(x y \otimes s_{1}\right) \otimes \iota_{1}=\left(x \otimes 1+\alpha\left(x \otimes s_{1}\right) \otimes \iota_{1}\right) \cdot\left(y \otimes 1+\alpha\left(y \otimes s_{1}\right) \otimes \iota_{1}\right)
$$

for $x, y \in H_{*}\left(T H H(R) ; \mathbb{F}_{p}\right)$. Multiplying out and comparing $\iota_{1}$-coefficients gives the claimed formulas.

We also wish to describe the $A_{*}$-comodule structure on $H_{*}\left(T H H(R) ; \mathbb{F}_{p}\right)$. In the cases $R=B P\langle m-1\rangle$ the following observations will suffice. The $A_{*}$ comodule coaction map

$$
\nu: H_{*}\left(R ; \mathbb{F}_{p}\right) \rightarrow A_{*} \otimes H_{*}\left(R ; \mathbb{F}_{p}\right)
$$

is in each case given by restricting the coproduct

$$
\psi: A_{*} \rightarrow A_{*} \otimes A_{*}
$$

given in Subsection 5.1, to the subalgebra $H_{*}\left(R ; \mathbb{F}_{p}\right) \subset A_{*}$, since the latter inclusion is induced by a spectrum map $R \rightarrow H \mathbb{F}_{p}$. The operator

$$
\sigma: H_{*}\left(\Sigma R ; \mathbb{F}_{p}\right) \rightarrow H_{*}\left(T H H(R) ; \mathbb{F}_{p}\right)
$$

is also induced by a spectrum map, hence is an $A_{*}$-comodule homomorphism. Hence the coaction map

$$
\nu: H_{*}\left(T H H(R) ; \mathbb{F}_{p}\right) \rightarrow A_{*} \otimes H_{*}\left(T H H(R) ; \mathbb{F}_{p}\right)
$$

satisfies

$$
\nu \circ \sigma=(1 \otimes \sigma) \nu
$$

As before, we know that the hypotheses of the following proposition are all satisfied in the commutative cases $0 \leq m \leq 2$, and for parts (a) and (c) in the $E_{4}$ ring spectrum case $m=\infty$. 
Theorem 5.12 (a) Let $p$ and $0 \leq m \leq \infty$ be such that $B P\langle m-1\rangle$ admits the structure of an $E_{3}$ ring spectrum. Then for $m<\infty$ we have

$$
\begin{aligned}
& H_{*}\left(T H H(B P\langle m-1\rangle) ; \mathbb{F}_{2}\right)= \\
& H_{*}\left(B P\langle m-1\rangle ; \mathbb{F}_{2}\right) \otimes E\left(\sigma \bar{\xi}_{1}^{2}, \ldots, \sigma \bar{\xi}_{m}^{2}\right) \otimes P\left(\sigma \bar{\xi}_{m+1}\right)
\end{aligned}
$$

when $p=2$, and

$$
\begin{aligned}
H_{*}\left(T H H(B P\langle m-1\rangle) ; \mathbb{F}_{p}\right) & = \\
& H_{*}\left(B P\langle m-1\rangle ; \mathbb{F}_{p}\right) \otimes E\left(\sigma \bar{\xi}_{1}, \ldots, \sigma \bar{\xi}_{m}\right) \otimes P\left(\sigma \bar{\tau}_{m}\right)
\end{aligned}
$$

when $p$ is odd, as $H_{*}\left(B P\langle m-1\rangle ; \mathbb{F}_{p}\right)$-algebras. For $m=\infty$ we have

$$
H_{*}\left(T H H(B P) ; \mathbb{F}_{2}\right)=H_{*}\left(B P ; \mathbb{F}_{2}\right) \otimes E\left(\sigma \bar{\xi}_{k}^{2} \mid k \geq 1\right)
$$

when $p=2$, and

$$
H_{*}\left(T H H(B P) ; \mathbb{F}_{p}\right)=H_{*}\left(B P ; \mathbb{F}_{p}\right) \otimes E\left(\sigma \bar{\xi}_{k} \mid k \geq 1\right)
$$

when $p$ is odd, as $H_{*}\left(B P ; \mathbb{F}_{p}\right)$-algebras.

(b) When $p$ and $m$ are such that $B P\langle m-1\rangle$ is a commutative $S$-algebra, then these are isomorphisms of primitively generated $H_{*}\left(B P\langle m-1\rangle ; \mathbb{F}_{p}\right)$-Hopf algebras.

(c) The $A_{*}$-comodule coaction on $H_{*}\left(\operatorname{THH}(B P\langle m-1\rangle) ; \mathbb{F}_{p}\right)$ is given on the tensor factor $H_{*}\left(B P\langle m-1\rangle ; \mathbb{F}_{p}\right)$ by restricting the coproduct on $A_{*}$. For $p=2$ the algebra generators $\sigma \bar{\xi}_{k}^{2}$ for $1 \leq k \leq m$ are $A_{*}$-comodule primitives, while

$$
\nu\left(\sigma \bar{\xi}_{m+1}\right)=1 \otimes \sigma \bar{\xi}_{m+1}+\bar{\xi}_{1} \otimes \sigma \bar{\xi}_{m}^{2} .
$$

For $p$ odd the algebra generators $\sigma \bar{\xi}_{k}$ for $1 \leq k \leq m$ are $A_{*}$-comodule primitives, while

$$
\nu\left(\sigma \bar{\tau}_{m}\right)=1 \otimes \sigma \bar{\tau}_{m}+\bar{\tau}_{0} \otimes \sigma \bar{\xi}_{m} .
$$

As usual, $\bar{\xi}_{0}$ is read as 1 in such formulas. Thus for $m=0, \sigma \bar{\xi}_{1}$ and $\sigma \bar{\tau}_{0}$ are also primitive.

Proof (a) We first resolve the algebra extensions in the $E^{\infty}$-terms from Proposition [5.7. In each case the Bökstedt spectral sequence is one of commutative $H_{*}\left(B P\langle m-1\rangle ; \mathbb{F}_{p}\right)$-algebras, by the $E_{3}$ ring spectrum hypothesis and Proposition 4.3. By the functoriality of the zeroth Postnikov section, the $S$ algebra maps $B P\langle m-1\rangle \rightarrow H \mathbb{Z}_{(p)} \rightarrow H \mathbb{F}_{p}$ and their composite are $E_{3}$ ring spectrum maps. (We only need this for $m \neq 0$.) Since the induced map in homology is injective, we can read off the $E_{3}$ ring spectrum Dyer-Lashof operations in $H_{*}\left(B P\langle m-1\rangle ; \mathbb{F}_{p}\right)$ from the formulas in $A_{*}=H_{*}\left(H \mathbb{F}_{p} ; \mathbb{F}_{p}\right)$. This 
tells us that $Q^{2^{k+1}-1}\left(\bar{\xi}_{k}^{2}\right)=0$ (by the Cartan formula) and $Q^{2^{k}}\left(\bar{\xi}_{k}\right)=\bar{\xi}_{k+1}$ for $p=2$, and that $Q^{p^{k}}\left(\bar{\tau}_{k}\right)=\bar{\tau}_{k+1}$ for $p$ odd.

We will also use the formulas $x^{2}=Q^{k}(x)$ for $|x|=k$ and $p=2$, and $x^{p}=Q^{k}(x)$ for $|x|=2 k$ and $p$ odd, from BMMS86, III.1.1(4)]. Likewise we will use the formulas $Q^{k}(\sigma x)=\sigma Q^{k}(x)$ for $|x|=k-1$ and $p=2$, and $Q^{k}(\sigma x)=\sigma Q^{k}(x)$ for $|x|=2 k-1$ and $p$ odd, from Proposition [5.9. These are all are valid in the homology of the $E_{2}$ ring spectrum $T H H(B P\langle m-1\rangle)$.

With these preliminaries, we are ready to compute. For $p=2$ the squares in $H_{*}\left(T H H(B P\langle m-1\rangle) ; \mathbb{F}_{2}\right)$ of the algebra generators in $E_{* *}^{\infty}(B P\langle m-1\rangle)$ are

$$
\left(\sigma \bar{\xi}_{k}^{2}\right)^{2}=Q^{2^{k+1}-1}\left(\sigma \bar{\xi}_{k}^{2}\right)=\sigma Q^{2^{k+1}-1}\left(\bar{\xi}_{k}^{2}\right)=0
$$

for $k=1, \ldots, m$ and

$$
\left(\sigma \bar{\xi}_{k}\right)^{2}=Q^{2^{k}}\left(\sigma \bar{\xi}_{k}\right)=\sigma Q^{2^{k}}\left(\bar{\xi}_{k}\right)=\sigma \bar{\xi}_{k+1}
$$

for $k \geq m+1$.

For $p$ odd the classes $\sigma \bar{\xi}_{k}$ remain exterior, since they are of odd degree in the graded commutative algebra $H_{*}\left(T H H(B P\langle m-1\rangle) ; \mathbb{F}_{p}\right)$. The $p$-th powers of the truncated polynomial generators in $E_{* *}^{\infty}(B P\langle m-1\rangle)$ are

$$
\left(\sigma \bar{\tau}_{k}\right)^{p}=Q^{p^{k}}\left(\sigma \bar{\tau}_{k}\right)=\sigma Q^{p^{k}}\left(\bar{\tau}_{k}\right)=\sigma \bar{\tau}_{k+1}
$$

for $k \geq m$. Hence these truncated polynomial algebras assemble to a polynomial algebra on the single generator $\sigma \bar{\tau}_{m}$.

(b) In the strictly commutative case, Proposition 4.9 tells us that all the algebra generators are coalgebra primitive, since they are of the form form $\sigma x$ in Hochschild filtration 1.

(c) To compute the $A_{*}$-comodule structure we use that $\sigma$ is a graded derivation, in view of Proposition 5.10 so $\sigma\left(y^{p}\right)=0$ and $\sigma(1)=0$. Then for $p=2$ and $1 \leq k \leq m$ we have

$$
\nu\left(\sigma \bar{\xi}_{k}^{2}\right)=\sum_{i+j=k} \bar{\xi}_{i}^{2} \otimes \sigma \bar{\xi}_{j}^{2+1}=1 \otimes \sigma \bar{\xi}_{k}^{2}
$$

while

$$
\nu\left(\sigma \bar{\xi}_{m+1}\right)=\sum_{i+j=m+1} \bar{\xi}_{i} \otimes \sigma \bar{\xi}_{j}^{2}=1 \otimes \sigma \bar{\xi}_{m+1}+\bar{\xi}_{1} \otimes \sigma \bar{\xi}_{m}^{2} .
$$

For $p$ odd and $1 \leq k \leq m$ we get

$$
\nu\left(\sigma \bar{\xi}_{k}\right)=\sum_{i+j=k} \bar{\xi}_{i} \otimes \sigma \bar{\xi}_{j}^{p^{i}}=1 \otimes \sigma \bar{\xi}_{k}
$$


while

$$
\nu\left(\sigma \bar{\tau}_{m}\right)=1 \otimes \sigma \bar{\tau}_{m}+\sum_{i+j=m} \bar{\tau}_{i} \otimes \sigma \bar{\xi}_{j}^{p}=1 \otimes \sigma \bar{\tau}_{m}+\bar{\tau}_{0} \otimes \sigma \bar{\xi}_{m}
$$

We can get similar conclusions under different hypotheses, by use of naturality with respect to the maps $B P \rightarrow B P\langle m-1\rangle \rightarrow H \mathbb{F}_{p}$. Strickland's result Str99, 2.10] suggests that it may be doable, but nontrivial, to relate a given $E_{2}$ ring spectrum structure on $B P\langle m-1\rangle$ to the one on $B P$ by such a structured map.

Corollary 5.13 Let $p$ and $2<m<\infty$ be such that there is an $E_{2}$ ring spectrum map $B P \rightarrow B P\langle m-1\rangle$. Then there is a split extension of associative $\mathbb{F}_{p}$-algebras

$$
\begin{aligned}
E\left(\sigma \bar{\xi}_{1}^{2}, \ldots, \sigma \bar{\xi}_{m}^{2}\right) & \rightarrow \\
H_{*}\left(T H H(B P\langle m-1\rangle) ; \mathbb{F}_{2}\right) & \rightarrow H_{*}\left(B P\langle m-1\rangle ; \mathbb{F}_{2}\right) \otimes P\left(\sigma \bar{\xi}_{m+1}\right)
\end{aligned}
$$

when $p=2$, and

$$
\begin{aligned}
E\left(\sigma \bar{\xi}_{1}, \ldots, \sigma \bar{\xi}_{m}\right) & \rightarrow \\
& H_{*}\left(T H H(B P\langle m-1\rangle) ; \mathbb{F}_{p}\right) \rightarrow H_{*}\left(B P\langle m-1\rangle ; \mathbb{F}_{p}\right) \otimes P\left(\sigma \bar{\tau}_{m}\right)
\end{aligned}
$$

when $p$ is odd.

If furthermore $H_{*}\left(T H H(B P\langle m-1\rangle) ; \mathbb{F}_{p}\right)$ is commutative, for which it suffices that $T H H(B P\langle m-1\rangle)$ is homotopy commutative, then these extensions are trivial and

$$
\begin{aligned}
& H_{*}\left(T H H(B P\langle m-1\rangle) ; \mathbb{F}_{2}\right)= \\
& H_{*}\left(B P\langle m-1\rangle ; \mathbb{F}_{2}\right) \otimes E\left(\sigma \bar{\xi}_{1}^{2}, \ldots, \sigma \bar{\xi}_{m}^{2}\right) \otimes P\left(\sigma \bar{\xi}_{m+1}\right)
\end{aligned}
$$

for $p=2$, and

$$
\begin{aligned}
H_{*}\left(T H H(B P\langle m-1\rangle) ; \mathbb{F}_{p}\right) & = \\
& H_{*}\left(B P\langle m-1\rangle ; \mathbb{F}_{p}\right) \otimes E\left(\sigma \bar{\xi}_{1}, \ldots, \sigma \bar{\xi}_{m}\right) \otimes P\left(\sigma \bar{\tau}_{m}\right)
\end{aligned}
$$

for $p$ odd, as commutative $H_{*}\left(B P\langle m-1\rangle ; \mathbb{F}_{p}\right)$-algebras.

Proof For brevity we write $R=B P\langle m-1\rangle$. The zeroth Postnikov section provides an $E_{2}$ ring spectrum map $R \rightarrow H \mathbb{Z}_{(p)}$, which we can continue to $H \mathbb{F}_{p}$. The $E_{2}$ ring spectrum maps $B P \rightarrow R \rightarrow H \mathbb{F}_{p}$ then induce $S$-algebra maps

$$
T H H(B P) \rightarrow T H H(R) \rightarrow T H H\left(H \mathbb{F}_{p}\right) .
$$


The Bökstedt spectral sequences at the two ends were determined in Theorem 5.12 as the cases $m=\infty$ and $m=0$, respectively: $H_{*}\left(T H H(B P) ; \mathbb{F}_{p}\right)=$ $H_{*}\left(B P ; \mathbb{F}_{p}\right) \otimes E\left(\sigma \bar{\xi}_{k} \mid k \geq 1\right)$ and $H_{*}\left(T H H\left(H \mathbb{F}_{p}\right) ; \mathbb{F}_{p}\right)=A_{*} \otimes P\left(\sigma \bar{\tau}_{0}\right)$, for $p$ odd. The case $p=2$ is similar, and will be omitted.

The left hand map embeds the exterior algebra $E\left(\sigma \bar{\xi}_{1}, \ldots, \sigma \bar{\xi}_{m}\right)$ as a graded commutative subalgebra of $H_{*}\left(T H H(R) ; \mathbb{F}_{p}\right)$. It maps trivially (by the augmentation) under the second map.

The right hand map embeds $H_{*}\left(R ; \mathbb{F}_{p}\right)$ in $A_{*}$. It also embeds the remaining factor $P_{p}\left(\sigma \bar{\tau}_{k} \mid k \geq m\right)$ in the Bökstedt $E^{\infty}$-term as the polynomial subalgebra of $P\left(\sigma \bar{\tau}_{0}\right)$ that is generated by the single class $\sigma \bar{\tau}_{m}=\left(\sigma \bar{\tau}_{0}\right)^{p^{m}}$. This uses the multiplicative relations $\left(\sigma \bar{\tau}_{k}\right)^{p}=\sigma \bar{\tau}_{k+1}$ for $k \geq 0$ in $H_{*}\left(T H H\left(H \mathbb{F}_{p}\right) ; \mathbb{F}_{p}\right)$.

The product of the unit map $R \rightarrow T H H(R)$ and the suspension operator $\sigma$ provide a splitting $H_{*}\left(R ; \mathbb{F}_{p}\right) \otimes P\left(\sigma \bar{\tau}_{m}\right) \rightarrow H_{*}\left(T H H(R) ; \mathbb{F}_{p}\right)$. In the commutative case, this splitting amounts to a trivialization.

From here on we only consider the topological Hochschild homology of strictly commutative $S$-algebras, not of $E_{n}$ ring spectra for any finite $n$.

For later reference we extract from Theorem $[5.12$ the following special cases, which correspond to $m=2$. Recall that $H_{*}\left(k u ; \mathbb{F}_{2}\right)=\left(A / / E_{1}\right)_{*} \subset A_{*}$ and $H_{*}\left(\ell ; \mathbb{F}_{p}\right)=\left(A / / E_{1}\right)_{*} \subset A_{*}$.

Corollary 5.14 (a) There is an isomorphism

$$
H_{*}\left(T H H(k u) ; \mathbb{F}_{2}\right) \cong H_{*}\left(k u ; \mathbb{F}_{2}\right) \otimes E\left(\sigma \bar{\xi}_{1}^{2}, \sigma \bar{\xi}_{2}^{2}\right) \otimes P\left(\sigma \bar{\xi}_{3}\right)
$$

of primitively generated $H_{*}\left(k u ; \mathbb{F}_{2}\right)$-Hopf algebras.

The $A_{*}$-comodule coaction $\nu: H_{*}\left(T H H(k u) ; \mathbb{F}_{2}\right) \rightarrow A_{*} \otimes H_{*}\left(T H H(k u) ; \mathbb{F}_{2}\right)$ is given on $H_{*}\left(k u ; \mathbb{F}_{2}\right)$ by restricting the coproduct $\psi: A_{*} \rightarrow A_{*} \otimes A_{*}$, and on the algebra generators by $\nu\left(\sigma \bar{\xi}_{1}^{2}\right)=1 \otimes \sigma \bar{\xi}_{1}^{2}, \nu\left(\sigma \bar{\xi}_{2}^{2}\right)=1 \otimes \sigma \bar{\xi}_{2}^{2}$ and

$$
\nu\left(\sigma \bar{\xi}_{3}\right)=1 \otimes \sigma \bar{\xi}_{3}+\bar{\xi}_{1} \otimes \sigma \bar{\xi}_{2}^{2} .
$$

(b) There is an isomorphism

$$
H_{*}\left(T H H(\ell) ; \mathbb{F}_{p}\right) \cong H_{*}\left(\ell ; \mathbb{F}_{p}\right) \otimes E\left(\sigma \bar{\xi}_{1}, \sigma \bar{\xi}_{2}\right) \otimes P\left(\sigma \bar{\tau}_{2}\right)
$$

of primitively generated $H_{*}\left(\ell ; \mathbb{F}_{p}\right)$-Hopf algebras.

The $A_{*}$-comodule coaction $\nu: H_{*}\left(T H H(\ell) ; \mathbb{F}_{p}\right) \rightarrow A_{*} \otimes H_{*}\left(T H H(\ell) ; \mathbb{F}_{p}\right)$ is given on $H_{*}\left(\ell ; \mathbb{F}_{p}\right)$ by restricting the coproduct $\psi: A_{*} \rightarrow A_{*} \otimes A_{*}$, and on the algebra generators by $\nu\left(\sigma \bar{\xi}_{1}\right)=1 \otimes \sigma \bar{\xi}_{1}, \nu\left(\sigma \bar{\xi}_{2}\right)=1 \otimes \sigma \bar{\xi}_{2}$ and

$$
\nu\left(\sigma \bar{\tau}_{2}\right)=1 \otimes \sigma \bar{\tau}_{2}+\bar{\tau}_{0} \otimes \sigma \bar{\xi}_{2} .
$$




\section{The higher real cases}

For $p=2$ there are a few more known examples of commutative $S$-algebras such that $H^{*}\left(R ; \mathbb{F}_{p}\right)$ is a cyclic $A$-module. Let $k o$ be the connective real $K$-theory spectrum, with $H^{*}\left(k o ; \mathbb{F}_{2}\right) \cong A / / A_{1}=A \otimes_{A_{1}} \mathbb{F}_{2}$, and let tmf be the HopkinsMahowald topological modular forms spectrum, with $H^{*}\left(t m f ; \mathbb{F}_{2}\right) \cong A / / A_{2}=$ $A \otimes_{A_{2}} \mathbb{F}_{2}$. See e.g. [Re01, 21.5]. Here $A_{n} \subset A$ is the subalgebra generated by $S q^{1}, \ldots, S q^{2^{n}}$, so $A_{1}$ has rank 8 and $A_{2}$ has rank 64 . It is well known that $k o$ is a commutative $S$-algebra, and in the case of tmf this is a consequence of the Hopkins-Miller theory, as being presented in the commutative case by Goerss and Hopkins.

Proposition 6.1 There are maps of commutative $S$-algebras $k o \rightarrow H \mathbb{F}_{2}$ and tmf $\rightarrow H \mathbb{F}_{2}$ that induce the following identifications:

$$
H_{*}\left(k o ; \mathbb{F}_{2}\right)=P\left(\bar{\xi}_{1}^{4}, \bar{\xi}_{2}^{2}, \bar{\xi}_{k} \mid k \geq 3\right)
$$

and

$$
H_{*}\left(t m f ; \mathbb{F}_{2}\right)=P\left(\bar{\xi}_{1}^{8}, \bar{\xi}_{2}^{4}, \bar{\xi}_{3}^{2}, \bar{\xi}_{k} \mid k \geq 4\right)
$$

Proof The maps can be obtained from the zeroth Postnikov sections $k o \rightarrow$ $H \mathbb{Z}$ and tmf $\rightarrow H \mathbb{Z}$. The homology computations are then immediate by dualization from $H^{*}\left(k o ; \mathbb{F}_{2}\right) \cong A / / A_{1}$, cf. [Sto63], and $H^{*}\left(t m f ; \mathbb{F}_{2}\right) \cong A / / A_{2}$.

We now follow the outline of Section 5. By Proposition 2.4 the $E^{2}$-terms of the respective Bökstedt spectral sequences are

$$
E_{* *}^{2}(k o)=H_{*}\left(k o ; \mathbb{F}_{2}\right) \otimes E\left(\sigma \bar{\xi}_{1}^{4}, \sigma \bar{\xi}_{2}^{2}, \sigma \bar{\xi}_{k} \mid k \geq 3\right)
$$

and

$$
E_{* *}^{2}(t m f)=H_{*}\left(t m f ; \mathbb{F}_{2}\right) \otimes E\left(\sigma \bar{\xi}_{1}^{8}, \sigma \bar{\xi}_{2}^{4}, \sigma \bar{\xi}_{3}^{2}, \sigma \bar{\xi}_{k} \mid k \geq 4\right) .
$$

By Corollary 4.4 both spectral sequences collapse at the $E^{2}$-term, so $E_{* *}^{2}(R)=$ $E_{* *}^{\infty}(R)$. To resolve the algebra extensions we use the Dyer-Lashof operations and Proposition 5.9. The squares in $H_{*}\left(T H H(k o) ; \mathbb{F}_{2}\right)$ of the algebra generators in $E_{* *}^{\infty}(k o)$ are

$$
\begin{aligned}
& \left(\sigma \bar{\xi}_{1}^{4}\right)^{2}=Q^{5}\left(\sigma \bar{\xi}_{1}^{4}\right)=\sigma Q^{5}\left(\bar{\xi}_{1}^{4}\right)=0 \\
& \left(\sigma \bar{\xi}_{2}^{2}\right)^{2}=Q^{7}\left(\sigma \bar{\xi}_{2}^{2}\right)=\sigma Q^{7}\left(\bar{\xi}_{2}^{2}\right)=0
\end{aligned}
$$

by the formula $Q^{k}\left(y^{2}\right)=0$ for $p=2$ and $k$ odd, and

$$
\left(\sigma \bar{\xi}_{k}\right)^{2}=Q^{2^{k}}\left(\sigma \bar{\xi}_{k}\right)=\sigma Q^{2^{k}}\left(\bar{\xi}_{k}\right)=\sigma \bar{\xi}_{k+1}
$$


for all $k \geq 3$. Similar calculations show that $\left(\sigma \bar{\xi}_{1}^{8}\right)^{2}=0,\left(\sigma \bar{\xi}_{2}^{4}\right)^{2}=0$ and $\left(\sigma \bar{\xi}_{3}^{2}\right)^{2}=0$ in $H_{*}\left(T H H(t m f) ; \mathbb{F}_{2}\right)$, while $\left(\sigma \bar{\xi}_{k}\right)^{2}=\sigma \bar{\xi}_{k+1}$ for all $k \geq 4$. The $A_{*}$-comodule coaction map $\nu$ on the resulting algebra generators is obtained from the coproduct on $A_{*}$ and formula (5.11), as in the proof of Theorem 5.12. The result is as follows:

Theorem 6.2 (a) There is an isomorphism

$$
H_{*}\left(T H H(k o) ; \mathbb{F}_{2}\right) \cong H_{*}\left(k o ; \mathbb{F}_{2}\right) \otimes E\left(\sigma \bar{\xi}_{1}^{4}, \sigma \bar{\xi}_{2}^{2}\right) \otimes P\left(\sigma \bar{\xi}_{3}\right)
$$

of primitively generated $H_{*}\left(k o ; \mathbb{F}_{2}\right)$-Hopf algebras. The $A_{*}$-comodule structure is given on $H_{*}\left(k o ; \mathbb{F}_{2}\right)$ by restricting the coproduct on $A_{*}$, and on the algebra generators by $\nu\left(\sigma \bar{\xi}_{1}^{4}\right)=1 \otimes \sigma \bar{\xi}_{1}^{4}$ and

$$
\begin{aligned}
& \nu\left(\sigma \bar{\xi}_{2}^{2}\right)=1 \otimes \sigma \bar{\xi}_{2}^{2}+\bar{\xi}_{1}^{2} \otimes \sigma \bar{\xi}_{1}^{4} \\
& \nu\left(\sigma \bar{\xi}_{3}\right)=1 \otimes \sigma \bar{\xi}_{3}+\bar{\xi}_{1} \otimes \sigma \bar{\xi}_{2}^{2}+\bar{\xi}_{2} \otimes \sigma \bar{\xi}_{1}^{4} .
\end{aligned}
$$

(b) There is an isomorphism

$$
H_{*}\left(T H H(t m f) ; \mathbb{F}_{2}\right) \cong H_{*}\left(t m f ; \mathbb{F}_{2}\right) \otimes E\left(\sigma \bar{\xi}_{1}^{8}, \sigma \bar{\xi}_{2}^{4}, \sigma \bar{\xi}_{3}^{2}\right) \otimes P\left(\sigma \bar{\xi}_{4}\right)
$$

of primitively generated $H_{*}\left(t m f ; \mathbb{F}_{2}\right)$-Hopf algebras. The $A_{*}$-comodule structure is given on $H_{*}\left(t m f ; \mathbb{F}_{2}\right)$ by restricting the coproduct on $A_{*}$, and on the algebra generators by $\nu\left(\sigma \bar{\xi}_{1}^{8}\right)=1 \otimes \sigma \bar{\xi}_{1}^{8}$ and

$$
\begin{aligned}
& \nu\left(\sigma \bar{\xi}_{2}^{4}\right)=1 \otimes \sigma \bar{\xi}_{2}^{4}+\bar{\xi}_{1}^{4} \otimes \sigma \bar{\xi}_{1}^{8} \\
& \nu\left(\sigma \bar{\xi}_{3}^{2}\right)=1 \otimes \sigma \bar{\xi}_{3}^{2}+\bar{\xi}_{1}^{2} \otimes \sigma \bar{\xi}_{2}^{4}+\bar{\xi}_{2}^{2} \otimes \sigma \bar{\xi}_{1}^{8} \\
& \nu\left(\sigma \bar{\xi}_{4}\right)=1 \otimes \sigma \bar{\xi}_{4}+\bar{\xi}_{1} \otimes \sigma \bar{\xi}_{3}^{2}+\bar{\xi}_{2} \otimes \sigma \bar{\xi}_{2}^{4}+\bar{\xi}_{3} \otimes \sigma \bar{\xi}_{1}^{8} .
\end{aligned}
$$

Proof Assemble the computations above.

\section{The real and complex image-of- $J$}

We now turn to the various image-of- $J$ spectra that are commutative $S$-algebras. Their mod $p$ cohomology is no longer cyclic as a module over the Steenrod algebra, but of rank 2, so extra work is needed to describe their homology as an $A_{*}$-comodule algebra. 


\subsection{The image-of- $J$ spectra}

For any prime $p$ let the $p$-local, connective complex image-of- $J$ spectrum be $j u=K\left(\mathbb{F}_{r}\right)_{(p)}$, where $r=3$ for $p=2$ and $r$ is a prime power that topologically generates the $p$-adic units for $p$ odd. Being the localized algebraic $K$-theory of a field, $j u$ is a commutative $S$-algebra May77, VIII.3.1]. For $p=2$ there is a cofiber sequence of spectra

$$
j u \stackrel{\kappa}{\longrightarrow} k u_{(2)} \stackrel{\psi^{3}-1}{\longrightarrow} b u_{(2)},
$$

where $b u \simeq \Sigma^{2} k u$ is the 1 -connected cover of $k u$ and $\psi^{3}$ is the Adams operation. For odd $p$ the cofiber sequence appears as

$$
j u \stackrel{\kappa}{\longrightarrow} \ell \stackrel{\psi^{r}-1}{\longrightarrow} \Sigma^{q} \ell
$$

with $q=2 p-2$, where $\ell \subset k u_{(p)}$ is the $p$-local, connective Adams summand and $\psi^{r}$ is the $r$-th Adams operation May77, V.5.16].

Let the 2-local, connective real image-of- $J$ spectrum be $j=K \mathcal{N}\left(\mathbb{F}_{3}\right)_{(2)}$, as defined in May77, VIII.3.1]. Being the localized algebraic $K$-theory of a symmetric bimonoidal category, $j$ is a commutative $S$-algebra. There is a cofiber sequence of spectra

$$
j \stackrel{\kappa}{\longrightarrow} k o_{(2)} \stackrel{\psi^{3}-1}{\longrightarrow} \operatorname{bspin}_{(2)},
$$

where bspin $\simeq \Sigma^{4} k s p$ is the 3-connected cover of $k o$ May77, V.5.16].

The fiber map $\kappa: j u \rightarrow \ell$ is a map of commutative $S$-algebras, at least after $p$-adic completion, because there is a discrete model $K\left(k^{\prime}\right)_{p}$ for $\ell_{p}$ with $k^{\prime}$ a suitable subfield of the algebraic closure of $\mathbb{F}_{r}$, and applying the functor $K(-)_{p}$

to the field inclusions $\mathbb{F}_{r} \subset k^{\prime} \subset \overline{\mathbb{F}}_{r}$ produces the commutative $S$-algebra maps

$$
j u_{p} \stackrel{\kappa}{\longrightarrow} \ell_{p} \subset k u_{p} .
$$

See [May77, VIII.3.2] and [MS93, §9]. Similarly, $\kappa: j \rightarrow k o$ becomes a map of commutative $S$-algebras after 2 -adic completion, since there is a discrete model $K \mathcal{O}\left(\overline{\mathbb{F}}_{3}\right)_{2}$ for $k o_{2}$, and $\kappa$ can be identified with the natural map

$$
K \mathcal{N}\left(\mathbb{F}_{3}\right)_{2} \rightarrow K \mathcal{O}\left(\overline{\mathbb{F}}_{3}\right)_{2}
$$

See May77, VIII.2.6 and 3.2]. 


\subsection{Cohomology modules}

Recall that

$$
H^{*}\left(\ell ; \mathbb{F}_{p}\right)=A / / E_{1} \cong \mathbb{F}_{p}\left\{1, P^{1}, \ldots, P^{p}, \ldots\right\},
$$

where $E_{1}=E\left(Q_{0}, Q_{1}\right) \subset A$ is the exterior algebra generated by $Q_{0}=\beta$ and $Q_{1}=\left[P^{1}, \beta\right]$, and that

$$
H^{*}\left(k o ; \mathbb{F}_{2}\right)=A / / A_{1} \cong \mathbb{F}_{2}\left\{1, S q^{4}, S q^{2} S q^{4} \equiv S q^{6}, S q^{1} S q^{2} S q^{4} \equiv S q^{7}, \ldots\right\},
$$

where $A_{1}=\left\langle S q^{1}, S q^{2}\right\rangle \subset A$ is the subalgebra generated by $S q^{1}$ and $S q^{2}$. There are also $A$-module isomorphisms

$$
\begin{aligned}
H^{*}\left(b o ; \mathbb{F}_{2}\right) & \cong \Sigma A / A S q^{2} \\
H^{*}\left(\text { bso } ; \mathbb{F}_{2}\right) & \cong \Sigma^{2} A / A S q^{3} \\
H^{*}\left(\text { bspin } ; \mathbb{F}_{2}\right) & \cong \Sigma^{4} A / A\left\{S q^{1}, S q^{2} S q^{3}\right\} .
\end{aligned}
$$

See [AP76, 2.5, 2.4 and p. 501]. Here $S q^{2} S q^{3}=S q^{5}+S q^{4} S q^{1}$ in admissible form, but the shorter expression is perhaps more memorable. For $p$ odd we let $A_{n} \subset A$ be the subalgebra generated by $\beta, P^{1}, \ldots, P^{p^{n-1}}$. In particular, $A_{1}=\left\langle\beta, P^{1}\right\rangle$ contains $E_{1}$, and

$$
A / / A_{1} \cong \mathbb{F}_{p}\left\{1, P^{p}, P^{1} P^{p} \equiv-P^{p+1}, \beta P^{1} P^{p} \equiv Q_{2}, \ldots\right\} .
$$

Lemma 7.6 (a) For $p=2$ the map $\psi^{3}-1: k u_{(2)} \rightarrow \Sigma^{2} k u_{(2)}$ induces right multiplication by $S q^{2}$ on $\bmod 2$ cohomology:

$$
\left(\psi^{3}-1\right)^{*}=S q^{2}: \Sigma^{2} A / / E_{1} \rightarrow A / / E_{1} .
$$

(b) For $p$ odd the map $\psi^{r}-1: \ell \rightarrow \Sigma^{q} \ell$ induces right multiplication by $P^{1}$ on mod $p$ cohomology:

$$
\left(\psi^{r}-1\right)^{*}=P^{1}: \Sigma^{q} A / / E_{1} \rightarrow A / / E_{1} .
$$

(c) For $p=2$ the map $\psi^{3}-1: k o_{(2)} \rightarrow \operatorname{bspin}_{(2)}$ induces right multiplication by $S q^{4}$ on mod 2 cohomology:

$$
\left(\psi^{3}-1\right)^{*}=S q^{4}: \Sigma^{4} A / A\left\{S q^{1}, S q^{2} S q^{3}\right\} \rightarrow A / / A_{1} .
$$

Case (c) is due to Mahowald and Milgram MaMi76, 3.4].

Proof The $S$-algebra unit map $e: S_{(p)} \rightarrow j u$ is well-known to be 2 -connected for $p=2$ and $(p q-2)$-connected for $p$ odd, since this is the degree of the first element $\beta_{1}$ in the $p$-primary cokernel of $J[\underline{\operatorname{Ra04}} 1.1 .14]$. Hence $e^{*}: H^{*}\left(j u ; \mathbb{F}_{p}\right) \rightarrow$ 
$H^{*}\left(S ; \mathbb{F}_{p}\right)=\mathbb{F}_{p}$ is cohomologically 2 -connected for $p=2$ and cohomologically $(p q-2)$-connected for $p$ odd, meaning that the homomorphism is injective in the stated degree and an isomorphism in lower degrees. In particular, $H^{2}\left(j u ; \mathbb{F}_{2}\right)=0$ for $p=2$ and $H^{q}\left(j u ; \mathbb{F}_{p}\right)=0$ for $p$ odd.

So in the long exact cohomology sequence

$$
\Sigma^{2} A / / E_{1} \stackrel{\left(\psi^{3}-1\right)^{*}}{\longrightarrow} A / / E_{1} \stackrel{\kappa^{*}}{\longrightarrow} H^{*}\left(j u ; \mathbb{F}_{2}\right)
$$

associated to the cofiber sequence (17.2), the non-zero class of $S q^{2}$ in $A / / E_{1}$ maps to zero under $\kappa^{*}$, hence is in the image of $\left(\psi^{3}-1\right)^{*}$. The latter is a left $A$-module homomorphism, and can only take $\Sigma^{2}(1)$ to $S q^{2}$, hence is given by right multiplication by $S q^{2}$. This proves (a). For part (b) we use the same argument for the exact sequence

$$
\Sigma^{q} A / / E_{1} \stackrel{\left(\psi^{r}-1\right)^{*}}{\longrightarrow} A / / E_{1} \stackrel{\kappa^{*}}{\longrightarrow} H^{*}\left(j u ; \mathbb{F}_{p}\right)
$$

associated to (7.3), in cohomological degree $q$. The non-zero class of $P^{1}$ in $A / / E_{1}$ maps to zero under $\kappa^{*}$, hence must equal the image of $\Sigma^{q}(1)$ under $\left(\psi^{r}-1\right)^{*}$. This proves (b).

The unit map $e: S_{(2)} \rightarrow j$ is likewise well-known to be 6-connected, since this is the degree of the first element $\nu^{2}$ in the 2-primary cokernel of $J$, so $e^{*}: H^{*}\left(j ; \mathbb{F}_{2}\right) \rightarrow H^{*}\left(S ; \mathbb{F}_{2}\right)=\mathbb{F}_{2}$ is cohomologically 6 -connected. In particular, $H^{4}\left(j ; \mathbb{F}_{2}\right)=0$. So in the long exact cohomology sequence associated to the cofiber sequence (7.4)

$$
\Sigma^{4} A / A\left\{S q^{1}, S q^{2} S q^{3}\right\} \stackrel{\left(\psi^{3}-1\right)^{*}}{\longrightarrow} A / / A_{1} \stackrel{\kappa^{*}}{\longrightarrow} H^{*}\left(j ; \mathbb{F}_{2}\right)
$$

the non-zero class of $S q^{4}$ in $A / / A_{1}$ maps to zero under $\kappa^{*}$, hence is in the image of $\left(\psi^{3}-1\right)^{*}$. The only class that can hit it is $\Sigma^{4}(1)$, which proves (c).

Lemma 7.10 (a) For $p=2$ there is a uniquely split extension of $A$-modules

$$
0 \rightarrow A / / A_{1} \rightarrow H^{*}\left(j u ; \mathbb{F}_{2}\right) \rightarrow \Sigma^{3} A / / A_{1} \rightarrow 0 .
$$

Hence there is a canonical $A$-module isomorphism $H^{*}\left(j u ; \mathbb{F}_{2}\right) \cong A / / A_{1}\{1, x\}$, with $x$ a class in degree 3 .

(b) For $p$ odd there is a non-split extension of $A$-modules

$$
0 \rightarrow A / / A_{1} \rightarrow H^{*}\left(j u ; \mathbb{F}_{p}\right) \rightarrow \Sigma^{p q-1} A / / A_{1} \rightarrow 0 .
$$

As an $A$-module, $H^{*}\left(j u ; \mathbb{F}_{p}\right)$ is generated by two classes 1 and $x$ in degrees 0 and $(p q-1)$, respectively, with $\beta(x)=P^{p}(1)$. 
(c) There is a unique non-split extension of $A$-modules

$$
0 \rightarrow A / / A_{2} \rightarrow H^{*}\left(j ; \mathbb{F}_{2}\right) \rightarrow A \otimes_{A_{2}} \Sigma^{7} K \rightarrow 0 .
$$

The cyclic $A_{2}$-module $K=A_{2} / A_{2}\left\{S q^{1}, S q^{7}, S q^{4} S q^{6}+S q^{6} S q^{4}\right\}$ has rank 17 over $\mathbb{F}_{2}$. As an $A$-module, $H^{*}\left(j ; \mathbb{F}_{2}\right)$ is generated by two classes 1 and $x$ in degrees 0 and 7 , respectively, with $S q^{1}(x)=S q^{8}(1)$.

For case (b), see also Rog03, 5.1(b)]. Case (c) is due to Davis Da75, Thm. 1], who also shows that $H^{*}\left(j ; \mathbb{F}_{2}\right)$ is a free $A / / A_{3}$-module.

Proof (a) In the long exact sequence extending (7.7) the $A$-module homomorphism $\left(\psi^{3}-1\right)^{*}$ is induced up from the $A_{1}$-module homomorphism

$$
S q^{2}: \Sigma^{2} A_{1} / / E_{1} \rightarrow A_{1} / / E_{1}=\mathbb{F}_{2}\left\{1, S q^{2}\right\}
$$

with kernel $\Sigma^{2} \mathbb{F}_{2}\left\{S q^{2}\right\}$ and cokernel $\mathbb{F}_{2}\{1\}$. Since $A$ is flat (in fact free) over $A_{1}$, it follows that $\operatorname{ker}\left(\psi^{3}-1\right)^{*} \cong \Sigma^{4} A / / A_{1}$ and $\operatorname{cok}\left(\psi^{3}-1\right)^{*} \cong A / / A_{1}$. Hence there is an extension of $A$-modules

$$
0 \rightarrow A / / A_{1} \rightarrow H^{*}\left(j u ; \mathbb{F}_{2}\right) \rightarrow \Sigma^{3} A / / A_{1} \rightarrow 0,
$$

as asserted. The group of such extensions is trivial, by the change-of-rings isomorphism

$$
\operatorname{Ext}_{A}^{1}\left(\Sigma^{3} A / / A_{1}, A / / A_{1}\right) \cong \operatorname{Ext}_{A_{1}}^{1}\left(\Sigma^{3} \mathbb{F}_{2}, A / / A_{1}\right) .
$$

For in any $A_{1}$-module extension

$$
0 \rightarrow A / / A_{1} \rightarrow E \rightarrow \Sigma^{3} \mathbb{F}_{2} \rightarrow 0
$$

let $x \in E$ be the unique class in degree 3 that maps to $\Sigma^{3}(1)$. Then $S q^{2} x=0$ since $A / / A_{1}=\mathbb{F}_{2}\left\{1, S q^{4}, S q^{6}, S q^{7}, \ldots\right\}$ is trivial in degree 5. Furthermore $S q^{1} x=S q^{4}(1)$ would contradict the Adem relation $S q^{2} S q^{2}=S q^{3} S q^{1}$, since $S q^{3} S q^{4} \equiv S q^{7}$ in $A / / A_{1}$. So $S q^{1} x=0$ and the extension $E$ is trivial.

Two choices of splitting maps for the trivial extension describing $H^{*}\left(j u ; \mathbb{F}_{2}\right)$ differ by an $A$-module homomorphism $\Sigma^{3} A / / A_{1} \rightarrow A / / A_{1}$, which must be zero since $A / / A_{1}$ is trivial in degree 3 . Therefore the splitting is unique, as claimed.

(b) Similarly, in (7.8) the $A$-module homomorphism $\left(\psi^{r}-1\right)^{*}$ is induced up from the $A_{1}$-module homomorphism

$$
P^{1}: \Sigma^{q} A_{1} / / E_{1} \rightarrow A_{1} / / E_{1}=\mathbb{F}_{p}\left\{1, P^{1}, \ldots, P^{p-1}\right\}
$$

with kernel $\Sigma^{q} \mathbb{F}_{p}\left\{P^{p-1}\right\}$ and cokernel $\mathbb{F}_{p}\{1\}$. As above it follows that $\operatorname{ker}\left(\psi^{r}-\right.$ $1)^{*} \cong \Sigma^{p q} A / / A_{1}$ and $\operatorname{cok}\left(\psi^{r}-1\right)^{*} \cong A / / A_{1}$. Hence there is an extension of $A$-modules

$$
0 \rightarrow A / / A_{1} \rightarrow H^{*}\left(j u ; \mathbb{F}_{p}\right) \rightarrow \Sigma^{p q-1} A / / A_{1} \rightarrow 0
$$


as asserted. This time the group of extensions is non-trivial; in fact it is isomorphic to $\mathbb{Z} / p$ and generated by the extension above. To see this, we again use the change-of-rings isomorphism

$$
\operatorname{Ext}_{A}^{1}\left(\Sigma^{p q-1} A / / A_{1}, A / / A_{1}\right) \cong \operatorname{Ext}_{A_{1}}^{1}\left(\Sigma^{p q-1} \mathbb{F}_{p}, A / / A_{1}\right)
$$

and consider $A_{1}$-module extensions

$$
0 \rightarrow A / / A_{1} \rightarrow E \rightarrow \Sigma^{p q-1} \mathbb{F}_{p} \rightarrow 0 .
$$

Let $x \in E$ be the unique class that maps to $\Sigma^{p q-1}(1)$. Then $P^{1} x=0$ since $A / / A_{1}=\mathbb{F}_{p}\left\{1, P^{p}, P^{1} P^{p}, \ldots\right\}$ is trivial in degree $(p+1) q-1$. But $\beta x$ is a multiple of $P^{p}$, and this multiple in $\mathbb{Z} / p$ classifies the extension.

To see that $\beta x$ is non-zero in the case of $H^{*}\left(j u ; \mathbb{F}_{p}\right)$, recall again that the first class $\beta_{1}$ in the cokernel of $J$ is in degree $(p q-2)$ and has order $p$. Let $c \rightarrow S_{(p)} \rightarrow j u$ be the usual cofiber sequence. Then by the Hurewicz and universal coefficient theorems, the lowest class $x$ in $H^{*}\left(\Sigma c ; \mathbb{F}_{p}\right)$ sits in degree $(p q-1)$ and supports a non-trivial mod $p$ Bockstein $\beta x \neq 0$. Furthermore, $H^{*}\left(\Sigma c ; \mathbb{F}_{p}\right) \cong H^{*}\left(j u ; \mathbb{F}_{p}\right)$ in positive degrees $*>0$, so also in $H^{*}\left(j u ; \mathbb{F}_{p}\right)$ we have $\beta x \neq 0$.

(c) In (7.9), the $A$-module homomorphism $\left(\psi^{3}-1\right)^{*}$ is induced up from the $A_{2}$-module homomorphism

$$
S q^{4}: \Sigma^{4} A_{2} / A_{2}\left\{S q^{1}, S q^{2} S q^{3}\right\} \rightarrow A_{2} / / A_{1} .
$$

A direct calculation shows that $A_{2} / A_{2}\left\{S q^{1}, S q^{2} S q^{3}\right\}$ has rank 24 and $A_{2} / / A_{1}$ has rank 8 , as $\mathbb{F}_{2}$-vector spaces. The cokernel of the homomorphism $S q^{4}$ is $A_{2} / / A_{2}=\mathbb{F}_{2}\{1\}$, of rank 1 , so its kernel $\Sigma^{8} K$ has rank 17 . Here

$$
\begin{gathered}
\Sigma^{4} K=\mathbb{F}_{2}\left\{S q^{4}, S q^{6}, S q^{7}, S q^{6} S q^{2}, S q^{9}, S q^{10}+S q^{8} S q^{2}, S q^{7} S q^{3},\right. \\
S q^{11}+S q^{9} S q^{2}, S q^{10} S q^{2}, S q^{13}+S q^{10} S q^{3}, S q^{11} S q^{2}, S q^{11} S q^{3}, \\
\left.S q^{13} S q^{2}+S q^{12} S q^{3}, S q^{13} S q^{3}, S q^{17}+S q^{15} S q^{2}, S q^{17} S q^{2}+S q^{16} S q^{3}, S q^{17} S q^{3}\right\}
\end{gathered}
$$

as a submodule of $A_{2} / A_{2}\left\{S q^{1}, S q^{2} S q^{3}\right\}$. By another direct calculation, $\Sigma^{4} K$ is in fact the cyclic $A_{2}$-submodule generated by $S q^{4}$. The annihilator ideal turns out to be generated by $S q^{1}, S q^{7}$ and $S q^{4} S q^{6}+S q^{6} S q^{4}=S q^{10}+S q^{8} S q^{2}+S q^{7} S q^{3}$ (in admissible form), so

$$
K \cong A_{2} / A_{2}\left\{S q^{1}, S q^{7}, S q^{4} S q^{6}+S q^{6} S q^{4}\right\}
$$

Hence there is an extension of $A$-modules

$$
0 \rightarrow A / / A_{2} \rightarrow H^{*}\left(j ; \mathbb{F}_{2}\right) \rightarrow A \otimes_{A_{2}} \Sigma^{7} K \rightarrow 0
$$


with $A \otimes_{A_{2}} \Sigma^{7} K \cong \Sigma^{7} A / A\left\{S q^{1}, S q^{7}, S q^{4} S q^{6}+S q^{6} S q^{4}\right\}$. The group of such $A$-module extensions is

$$
\operatorname{Ext}_{A}^{1}\left(A \otimes A_{2} \Sigma^{7} K, A / / A_{2}\right) \cong \mathbb{Z} / 2,
$$

and the extension is determined by the action of $S q^{1}$ on the generator $x$ in degree 7 that maps to $\Sigma^{7}(1) .\left(S q^{7} x=0\right.$ by the Adem relation $S q^{1} S q^{7}=0$ and the fact that $S q^{1}$ acts injectively from degree 14 of $A / / A_{2}$. $\left(S q^{4} S q^{6}+\right.$ $\left.S q^{6} S q^{4}\right) x=0$ since $A / / A_{2}$ is trivial in degree 17.)

To see that $S q^{1}(x)=S q^{8}(1) \neq 0$ in $H^{*}(j ; \mathbb{Z} / 2)$, we once again use the cofiber sequence $c \rightarrow S_{(2)} \rightarrow j$ and the fact that $c$ is 5 -connected with $\pi_{6}(c)=$ $\mathbb{Z} / 2\left\{\nu^{2}\right\}$. Hence the lowest class $x$ in $H^{*}\left(\Sigma c ; \mathbb{F}_{2}\right)$ sits in degree 7 and supports a non-trivial $S q^{1} x \neq 0$. Again, $H^{*}\left(\Sigma c ; \mathbb{F}_{2}\right) \cong H^{*}\left(j ; \mathbb{F}_{2}\right)$ in positive degrees, so also in $H^{*}\left(j ; \mathbb{F}_{2}\right)$ we have $S q^{1} x \neq 0$. The only possible nonzero value is the $S q^{8}$ from $A / / A_{2}$.

We display the $A_{2}$-module $\Sigma^{4} K \subset A_{2} / A_{2}\left\{S q^{1}, S q^{2} S q^{3}\right\}$ below. Here $(i)$ or $(i, j)$ denotes an admissible class with lexicographically leading term $S q^{i}$ or $S q^{i} S q^{j}$, respectively. The arrows indicate the $S q^{1}$ - and $S q^{2}$-operations. The $S q^{4}$-operations can be deduced from the relations $S q^{4}(6)=(10)$ and $S q^{4}(13)=$ (17), but are omitted to avoid cluttering the diagram.

(4)

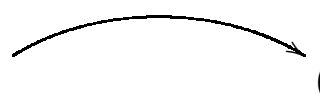

$(6)$
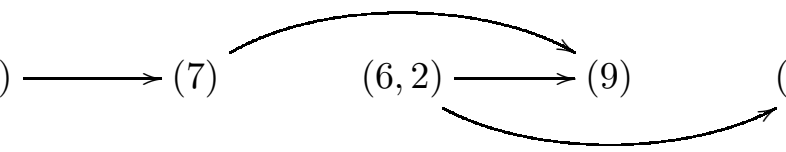

(10)

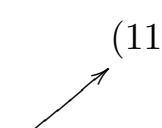

$(11)$

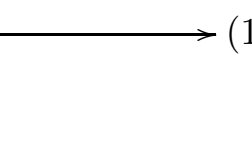

$(13)$
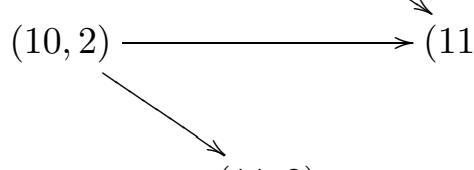

$11,3)$

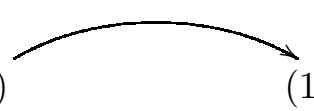

$(17,2) \longrightarrow(17,3)$

\subsection{Homology algebras}

Let us write $\left(A / / A_{1}\right)_{*} \subset A_{*}$ for the $A_{*}$-comodule subalgebra dual to the quotient $A$-module coalgebra $A / / A_{1}$ of $A$. For $p=2$ we recall from Proposition 6.1 
that

$$
\left(A / / A_{1}\right)_{*}=P\left(\bar{\xi}_{1}^{4}, \bar{\xi}_{2}^{2}, \bar{\xi}_{k} \mid k \geq 3\right) \cong H_{*}\left(k o ; \mathbb{F}_{2}\right)
$$

For $p$ odd

$$
\left(A / / A_{1}\right)_{*}=P\left(\bar{\xi}_{1}^{p}, \bar{\xi}_{k} \mid k \geq 2\right) \otimes E\left(\bar{\tau}_{k} \mid k \geq 2\right),
$$

but there is no spectrum with $\bmod p$ homology realizing $\left(A / / A_{1}\right)_{*}$. For $p=2$ and for $p$ odd there are extensions of $A_{*}$-comodules

$$
0 \rightarrow \Sigma^{3}\left(A / / A_{1}\right)_{*} \rightarrow H_{*}\left(j u ; \mathbb{F}_{2}\right) \stackrel{\kappa}{\longrightarrow}\left(A / / A_{1}\right)_{*} \rightarrow 0
$$

and

$$
0 \rightarrow \Sigma^{p q-1}\left(A / / A_{1}\right)_{*} \rightarrow H_{*}\left(j u ; \mathbb{F}_{p}\right) \stackrel{\kappa}{\longrightarrow}\left(A / / A_{1}\right)_{*} \rightarrow 0,
$$

dual to the $A$-module extensions of Lemma 7.10(a) and (b), respectively, where in both cases $\kappa$ is an $A_{*}$-comodule algebra homomorphism.

Likewise, we write $\left(A / / A_{2}\right)_{*} \subset A_{*}$ for the $A_{*}$-comodule subalgebra dual to the quotient $A$-module coalgebra $A / / A_{2}$ of $A$. For $p=2$ we recall from Proposition 6.1 that

$$
\left(A / / A_{2}\right)_{*}=P\left(\bar{\xi}_{1}^{8}, \bar{\xi}_{2}^{4}, \bar{\xi}_{3}^{2}, \bar{\xi}_{k} \mid k \geq 4\right) \cong H_{*}\left(t m f ; \mathbb{F}_{2}\right) .
$$

There is an extension of $A_{*}$-comodules

$$
0 \rightarrow A_{*} \square_{A_{2 *}} \Sigma^{7} K_{*} \rightarrow H_{*}\left(j ; \mathbb{F}_{2}\right) \stackrel{\kappa}{\longrightarrow}\left(A / / A_{2}\right)_{*} \rightarrow 0,
$$

dual to the $A$-module extension of Lemma 7.10)(c), where $\kappa$ is an $A_{*}$-comodule algebra homomorphism. Here $K_{*} \subset A_{2 *}$ is the $A_{2 *}$-comodule dual to the cyclic $A_{2}$-module $K=A_{2} / A_{2}\left\{S q^{1}, S q^{7}, S q^{4} S q^{6}+S q^{6} S q^{4}\right\}$.

Proposition 7.12 (a) For $p=2$, let $b \in H_{3}\left(j u ; \mathbb{F}_{2}\right)$ be the image of $\Sigma^{3}(1)$ in $\Sigma^{3}\left(A / / A_{1}\right)_{*}$. Then there is an $A_{*}$-comodule algebra isomorphism

$$
H_{*}\left(j u ; \mathbb{F}_{2}\right) \cong\left(A / / A_{1}\right)_{*} \otimes E(b),
$$

where $\left(A / / A_{1}\right)_{*}$ has the subalgebra structure from $A_{*}$ and $b$ is $A_{*}$-comodule primitive.

The Dyer-Lashof operations in $H_{*}\left(j u ; \mathbb{F}_{2}\right)$ satisfy $Q^{4}(b)=0, Q^{5}\left(\bar{\xi}_{1}^{4}\right)=0$, $Q^{7}\left(\bar{\xi}_{2}^{2}\right)=0$ and $Q^{2^{k}}\left(\bar{\xi}_{k}\right)=\bar{\xi}_{k+1}$ for all $k \geq 3$, so $H_{*}\left(j u ; \mathbb{F}_{2}\right)$ is generated by $\bar{\xi}_{1}^{4}, \bar{\xi}_{2}^{2}, \bar{\xi}_{3}$ and $b$ as an algebra over the Dyer-Lashof algebra.

(b) For $p$ odd, let $b \in H_{p q-1}\left(j u ; \mathbb{F}_{p}\right)$ be the image of $\Sigma^{p q-1}(1)$ in $\Sigma^{p q-1}\left(A / / A_{1}\right)_{*}$. There is an algebra isomorphism

$$
\left(A / / A_{1}\right)_{*} \otimes E(b) \cong H_{*}\left(j u ; \mathbb{F}_{p}\right)
$$


that takes the algebra generators $\bar{\xi}_{1}^{p}, \bar{\xi}_{k}, \bar{\tau}_{k}$ (for $k \geq 2$ ) and $b$ to classes $\tilde{\xi}_{1}^{p}$, $\tilde{\xi}_{k}, \tilde{\tau}_{k}$ and $b$ in $H_{*}\left(j u ; \mathbb{F}_{p}\right)$, respectively. These map under $\kappa$ to $\bar{\xi}_{1}^{p}, \bar{\xi}_{k}, \bar{\tau}_{k}$ and 0 , respectively.

The Dyer-Lashof operations on $H_{*}\left(j u ; \mathbb{F}_{p}\right)$ satisfy $Q^{p q / 2}(b)=0$ and $Q^{p^{k}}\left(\tilde{\tau}_{k}\right)=$ $\tilde{\tau}_{k+1}$ for all $k \geq 2$, and $\beta\left(\tilde{\tau}_{k}\right)=\tilde{\xi}_{k}$ for all $k \geq 2$. Thus $H_{*}\left(j u ; \mathbb{F}_{p}\right)$ is generated as an algebra over the Dyer-Lashof algebra by $\tilde{\xi}_{1}^{p}, \tilde{\xi}_{2}, \tilde{\tau}_{2}$ and $b$.

The $A_{*}$-comodule structure is determined by

$$
\begin{aligned}
\nu(b) & =1 \otimes b \\
\nu\left(\tilde{\xi}_{1}^{p}\right) & =1 \otimes \tilde{\xi}_{1}^{p}-\tau_{0} \otimes b+\bar{\xi}_{1}^{p} \otimes 1 \\
\nu\left(\tilde{\xi}_{2}\right) & =1 \otimes \tilde{\xi}_{2}+\bar{\xi}_{1} \otimes \tilde{\xi}_{1}^{p}+\tau_{1} \otimes b+\bar{\xi}_{2} \otimes 1 \\
\nu\left(\tilde{\tau}_{2}\right) & =1 \otimes \tilde{\tau}_{2}+\bar{\tau}_{0} \otimes \tilde{\xi}_{2}+\bar{\tau}_{1} \otimes \tilde{\xi}_{1}^{p}-\tau_{0} \tau_{1} \otimes b+\bar{\tau}_{2} \otimes 1 .
\end{aligned}
$$

(The class $b \in H_{p q-1}\left(j u ; \mathbb{F}_{p}\right)$ maps under the connecting map for the cofiber sequence $c \rightarrow S_{(p)} \rightarrow j u$ to the mod $p$ Hurewicz image of $\beta_{1} \in \pi_{p q-2}(c)$, so the letter $b$ is chosen to correspond to $\beta$.)

(c) There is a square-zero extension of $A_{*}$-comodule algebras

$$
0 \rightarrow A_{*} \square_{A_{2 *}} \Sigma^{7} K_{*} \rightarrow H_{*}\left(j ; \mathbb{F}_{2}\right) \stackrel{\kappa}{\longrightarrow}\left(A / / A_{2}\right)_{*} \rightarrow 0,
$$

where $\kappa$ is split as an algebra homomorphism. As an $\left(A / / A_{2}\right)_{*}$-module,

$$
\operatorname{ker}(\kappa)=A_{*} \square_{A_{2 *}} \Sigma^{7} K_{*} \cong\left(A / / A_{2}\right)_{*} \otimes \Sigma^{7} K_{*}
$$

is free of rank 17. There is an algebra isomorphism

$$
H_{*}\left(j ; \mathbb{F}_{2}\right) \cong\left(A / / A_{2}\right)_{*} \otimes\left(\mathbb{F}_{2} \oplus \Sigma^{7} K_{*}\right)
$$

where $\mathbb{F}_{2} \oplus \Sigma^{7} K_{*}$ is the split square-zero extension of $\mathbb{F}_{2}$ with kernel $\Sigma^{7} K_{*}$ of rank 17.

Proof (a) Let $x \in H^{3}\left(j u ; \mathbb{F}_{2}\right)$ be the class that maps to $\Sigma^{3}(1)$ in the uniquely split $A$-module extension of Lemma 7.10(a). Then $\psi(x)=x \otimes 1+1 \otimes x$ since $H^{*}\left(j u ; \mathbb{F}_{2}\right)=0$ for $0<*<3$, so $E(x)=\mathbb{F}_{2}\{1, x\}$ (no algebra structure is implied) is a sub-coalgebra of $H^{*}\left(j u ; \mathbb{F}_{2}\right)$ and there a surjective composite $A$-module coalgebra homomorphism

$$
A \otimes E(x) \rightarrow A \otimes H^{*}\left(j u ; \mathbb{F}_{2}\right) \rightarrow H^{*}\left(j u ; \mathbb{F}_{2}\right) .
$$

Since the $A$-module extension is split, the generators $S q^{1}$ and $S q^{2}$ of $A_{1}$ act trivially on 1 and $x$ in $H^{*}\left(j u ; \mathbb{F}_{2}\right)$, so the surjection factors through a surjection

$$
A / / A_{1} \otimes E(x) \rightarrow H^{*}\left(j u ; \mathbb{F}_{2}\right),
$$


which by a dimension count must be an $A$-module coalgebra isomorphism.

Dually, let $b \in H_{3}\left(j u ; \mathbb{F}_{2}\right)$ be the image of $\Sigma^{3}(1)$ in the split $A_{*}$-comodule extension

$$
0 \rightarrow \Sigma^{3}\left(A / / A_{1}\right)_{*} \rightarrow H_{*}\left(j u ; \mathbb{F}_{2}\right) \stackrel{\kappa}{\longrightarrow}\left(A / / A_{1}\right)_{*} \rightarrow 0 .
$$

Then $b$ is dual to $x$, and the dual of the above isomorphism is an $A_{*}$-comodule algebra isomorphism

$$
H_{*}\left(j u ; \mathbb{F}_{2}\right) \cong\left(A / / A_{1}\right)_{*} \otimes E(b) .
$$

In particular, the unique $A_{*}$-comodule splitting $\left(A / / A_{1}\right)_{*} \rightarrow H_{*}\left(j u ; \mathbb{F}_{2}\right)$ is an algebra map, $b$ is an $A_{*}$-comodule primitive, and $b^{2}=0$.

To determine the Dyer-Lashof operations that we will need in $H_{*}\left(j u ; \mathbb{F}_{2}\right)$ we make use of the Nishida relations and the known $A_{*}$-comodule structure. Some of the Nishida relations that we shall use are

$$
S q_{*}^{1} Q^{s}= \begin{cases}Q^{s-1} & \text { for } s \text { even } \\ 0 & \text { for } s \text { odd }\end{cases}
$$

and

$$
S q_{*}^{2} Q^{s}=\left\{\begin{array}{lll}
Q^{s-2}+Q^{s-1} S q_{*}^{1} & \text { for } s \equiv 0,1 & \bmod 4 \\
Q^{s-1} S q_{*}^{1} & \text { for } s \equiv 2,3 & \bmod 4 .
\end{array}\right.
$$

See [BMMS86, III.1.1(8)].

The Dyer-Lashof operation $Q^{4}(b)$ lands in $H_{7}\left(j u ; \mathbb{F}_{2}\right) \cong \mathbb{F}_{2}\left\{\bar{\xi}_{3}, \bar{\xi}_{1}^{4} b\right\}$. From the $A_{*}$-comodule structure we can read off the dual Steenrod operations $S q_{*}^{1}\left(\bar{\xi}_{3}\right)=$ $\bar{\xi}_{2}^{2}$ and $S q_{*}^{4}\left(\bar{\xi}_{1}^{4} b\right)=b$, since $S q^{i}$ is dual to $\xi_{1}^{i}$. These are linearly independent, so $Q^{4}(b)$ is determined by its images under $S q_{*}^{1}$ and $S q_{*}^{4}$. By a Nishida relation we get that $S q_{*}^{1} Q^{4}(b)=Q^{3}(b)$, and $Q^{3}(b)=b^{2}=0$ since $|b|=3$ and $b$ is an exterior class. By another Nishida relation $S q_{*}^{4} Q^{4}(b)=Q^{2} S q_{*}^{2}(b)$, and $S q_{*}^{2}(b)=$ 0 since $b$ is $A_{*}$-comodule primitive. Thus $S q_{*}^{1} Q^{4}(b)=0$ and $S q_{*}^{4} Q^{4}(b)=0$, and the only possibility is that $Q^{4}(b)=0$.

The operation $Q^{5}\left(\bar{\xi}_{1}^{4}\right)$ lands in $H_{9}\left(j u ; \mathbb{F}_{2}\right) \cong \mathbb{F}_{2}\left\{\bar{\xi}_{2}^{2} b\right\}$. By a Nishida relation $S q_{*}^{2} Q^{5}\left(\bar{\xi}_{1}^{4}\right)=\left(Q^{3}+Q^{4} S q_{*}^{1}\right)\left(\bar{\xi}_{1}^{4}\right)=0$, while $S q_{*}^{2}\left(\bar{\xi}_{2}^{2} b\right)=\bar{\xi}_{1}^{4} b \neq 0$. So $Q^{5}\left(\bar{\xi}_{1}^{4}\right)$ must be zero.

The operation $Q^{7}\left(\bar{\xi}_{2}^{2}\right)$ lands in $H_{13}\left(j u ; \mathbb{F}_{2}\right) \cong \mathbb{F}_{2}\left\{\bar{\xi}_{2}^{2} \bar{\xi}_{3}, \bar{\xi}_{1}^{4} \bar{\xi}_{2}^{2} b\right\}$. Its image under $\kappa$ in $H_{*}\left(k u ; \mathbb{F}_{2}\right) \subset A_{*}$ must vanish, by the Cartan formula, so in fact $Q^{7}\left(\bar{\xi}_{2}^{2}\right) \in \mathbb{F}_{2}\left\{\bar{\xi}_{1}^{4} \bar{\xi}_{2}^{2} b\right\}$. By a Nishida relation $S q_{*}^{2} Q^{7}\left(\bar{\xi}_{2}^{2}\right)=Q^{6} S q_{*}^{1}\left(\bar{\xi}_{2}^{2}\right)=0$, while $S q_{*}^{2}\left(\bar{\xi}_{1}^{4} \bar{\xi}_{2}^{2} b\right)=\left(\bar{\xi}_{1}^{4}\right)^{2} b \neq 0$, so $Q^{7}\left(\bar{\xi}_{2}^{2}\right)$ must be zero. 
The claim that $Q^{2^{k}}\left(\bar{\xi}_{k}\right)=\bar{\xi}_{k+1}$ follows in the same way as in $A_{*}=H_{*}\left(H \mathbb{F}_{2} ; \mathbb{F}_{2}\right)$, see BMMS86, §III.6]. It suffices to show that $S q_{*}^{2^{m}} Q^{2^{k}}\left(\bar{\xi}_{k}\right)=S q_{*}^{2^{m}}\left(\bar{\xi}_{k+1}\right)$ for $0 \leq m \leq k$, because the only $A_{*}$-comodule primitives in $H_{*}\left(j u ; \mathbb{F}_{2}\right)$ are 1 and $b$. The right hand side equals $\bar{\xi}_{k}^{2}$ for $m=0$, and is zero otherwise, by the formula for $\psi\left(\bar{\xi}_{k+1}\right)$. The Nishida relations imply that $S q_{*}^{1} Q^{2^{k}}\left(\bar{\xi}_{k}\right)=Q^{2^{k}-1}\left(\bar{\xi}_{k}\right)=\bar{\xi}_{k}^{2}$, while $S q_{*}^{2} Q^{2^{k}}\left(\bar{\xi}_{k}\right)=Q^{2^{k}-1} S q_{*}^{1}\left(\bar{\xi}_{k}\right)=Q^{2^{k}-1}\left(\bar{\xi}_{k-1}^{2}\right)=0$ by the Cartan formula. For $2 \leq m \leq k$ we have $S q_{*}^{2^{m}} Q^{2^{k}}\left(\bar{\xi}_{k}\right)=Q^{2^{k}-2^{m-1}} S q_{*}^{2^{m-1}}\left(\bar{\xi}_{k}\right)=Q^{2^{k}-2^{m-1}}(0)=$ 0 by the formula for $\psi\left(\bar{\xi}_{k}\right)$ in $A_{*}$. Hence $Q^{2^{k}}\left(\bar{\xi}_{k}\right)=\bar{\xi}_{k+1}$ in $H_{*}\left(j u ; \mathbb{F}_{2}\right)$ is the only possibility.

(b) Let $x \in H^{p q-1}\left(j u ; \mathbb{F}_{p}\right)$ be the class that maps to $\Sigma^{p q-1}(1)$ in the $A$-module extension of Lemma 7.10(b). Then $\psi(x)=x \otimes 1+1 \otimes x$ since $H^{*}\left(j u ; \mathbb{F}_{p}\right)=0$ for $0<*<p q-1$, so $E(x)=\mathbb{F}_{p}\{1, x\}$ is a sub-coalgebra of $H^{*}\left(j u ; \mathbb{F}_{p}\right)$ and there a surjective composite $A$-module coalgebra homomorphism

$$
A \otimes E(x) \rightarrow A \otimes H^{*}\left(j u ; \mathbb{F}_{p}\right) \rightarrow H^{*}\left(j u ; \mathbb{F}_{p}\right) .
$$

Dually, let $b \in H_{p q-1}\left(j u ; \mathbb{F}_{p}\right)$ be the image of $\Sigma^{p q-1}(1)$ in the $A_{*}$-comodule extension

$$
0 \rightarrow \Sigma^{p q-1}\left(A / / A_{1}\right)_{*} \rightarrow H_{*}\left(j u ; \mathbb{F}_{p}\right) \stackrel{\kappa}{\longrightarrow}\left(A / / A_{1}\right)_{*} \rightarrow 0 .
$$

Then $E(b)$ is a quotient algebra of $H_{*}\left(j u ; \mathbb{F}_{p}\right)$ and the dual of the surjection above is an injective $A_{*}$-comodule algebra homomorphism

$$
H_{*}\left(j u ; \mathbb{F}_{p}\right) \rightarrow A_{*} \otimes E(b) .
$$

We shall describe $H_{*}\left(j u ; \mathbb{F}_{p}\right)$ in terms of its image under this injection.

Since $\left(A / / A_{1}\right)_{*}$ is a free graded commutative algebra, we can choose an algebra section $s:\left(A / / A_{1}\right)_{*} \rightarrow H_{*}\left(j u ; \mathbb{F}_{p}\right)$ to the surjection $\kappa: H_{*}\left(j u ; \mathbb{F}_{p}\right) \rightarrow\left(A / / A_{1}\right)_{*}$. We write $\tilde{\xi}_{1}^{p}=s\left(\bar{\xi}_{1}^{p}\right), \tilde{\xi}_{k}=s\left(\bar{\xi}_{k}\right)$ and $\tilde{\tau}_{k}=s\left(\bar{\tau}_{k}\right)$ for the lifted classes in $H_{*}\left(j u ; \mathbb{F}_{p}\right)$. Since $\Sigma^{p q-1}\left(A / / A_{1}\right)$ vanishes in the degrees of $\bar{\xi}_{1}^{p}$ and $\bar{\tau}_{2}$, the respective lifts $\tilde{\xi}_{1}^{p}$ and $\tilde{\tau}_{2}$ are unique, and we can use the commutative $S$-algebra structure on $j u$ and the resulting Dyer-Lashof operations on $H_{*}\left(j u ; \mathbb{F}_{p}\right)$ to fix the other lifts by the formulas $\tilde{\xi}_{k}=\beta\left(\tilde{\tau}_{k}\right)$ and $\tilde{\tau}_{k+1}=Q^{p^{k}}\left(\tilde{\tau}_{k}\right)$ for $k \geq 2$. This specifies the algebra section $s$ uniquely. (But beware, $s$ is not an $A_{*}$-comodule homomorphism!)

Since $|b|=p q-1$ is odd we have $b^{2}=0$ and the exterior algebra $E(b)$ is a subalgebra of $H_{*}\left(j u ; \mathbb{F}_{p}\right)$. Writing $i: E(b) \rightarrow H_{*}\left(j u ; \mathbb{F}_{p}\right)$ for the inclusion, we obtain an algebra map

$$
s \otimes i:\left(A / / A_{1}\right)_{*} \otimes E(b) \rightarrow H_{*}\left(j u ; \mathbb{F}_{p}\right),
$$


which we claim is an isomorphism. By a dimension count it suffices to show that its composite with the injection $H_{*}\left(j u ; \mathbb{F}_{p}\right) \rightarrow A_{*} \otimes E(b)$ is injective. We have a diagram of algebra maps

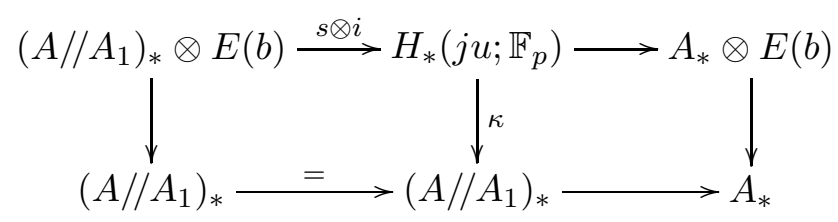

where the vertical maps take $b$ to zero. The lower map takes $\xi \in\left(A / / A_{1}\right)_{*}$ to $\xi \in A_{*}$, so the upper composite takes $\xi \otimes 1$ to $\xi \otimes 1(\bmod b)$. Hence the latter takes $\xi \otimes b$ to $\xi \otimes b\left(\bmod b^{2}=0\right)$, and it follows that the upper composite indeed is injective.

In low degrees, $H^{*}\left(j u ; \mathbb{F}_{p}\right) \cong \mathbb{F}_{p}\left\{1, x, P^{p}(1), P^{1} P^{p}(1), \beta P^{1} P^{p}(1), \ldots\right\}$ is dual to $H_{*}\left(j u ; \mathbb{F}_{p}\right) \cong \mathbb{F}_{p}\left\{1, b,-\tilde{\xi}_{1}^{p}, \tilde{\xi}_{2}, \tilde{\tau}_{2}, \ldots\right\}$. By Lemma 7.10 (b) we have $\beta(x)=$ $P^{p}(1)$, so dually the $A_{*}$-comodule coactions are given by

$$
\begin{aligned}
\nu(b) & =1 \otimes b \\
\nu\left(\tilde{\xi}_{1}^{p}\right) & =1 \otimes \tilde{\xi}_{1}^{p}-\tau_{0} \otimes b+\bar{\xi}_{1}^{p} \otimes 1 \\
\nu\left(\tilde{\xi}_{2}\right) & =1 \otimes \tilde{\xi}_{2}+\bar{\xi}_{1} \otimes \tilde{\xi}_{1}^{p}+\tau_{1} \otimes b+\bar{\xi}_{2} \otimes 1 \\
\nu\left(\tilde{\tau}_{2}\right) & =1 \otimes \tilde{\tau}_{2}+\bar{\tau}_{0} \otimes \tilde{\xi}_{2}+\bar{\tau}_{1} \otimes \tilde{\xi}_{1}^{p}-\tau_{0} \tau_{1} \otimes b+\bar{\tau}_{2} \otimes 1 .
\end{aligned}
$$

In particular, the images in $A_{*} \otimes E(b)$ of these classes are $1 \otimes b,-\tau_{0} \otimes b+\bar{\xi}_{1}^{p} \otimes 1$, $\tau_{1} \otimes b+\bar{\xi}_{2} \otimes 1$ and $-\tau_{0} \tau_{1} \otimes b+\bar{\tau}_{2} \otimes 1$, respectively.

The Dyer-Lashof operation $Q^{p q / 2}(b)$ lands in $H_{p^{2} q-1}\left(j u ; \mathbb{F}_{p}\right)=\mathbb{F}_{p}\left\{\tilde{\xi}_{1}^{p q / 2} b\right\}$. Here $P_{*}^{p q / 2}\left(\tilde{\xi}_{1}^{p q / 2} b\right)=b$ is non-zero, in view of the formula above for $\nu\left(\tilde{\xi}_{1}^{p}\right)$. By a Nishida relation $P_{*}^{p q / 2} Q^{p q / 2}(b)=Q^{q / 2} P_{*}^{q / 2}(b)=0$, so $Q^{p q / 2}(b)=0$.

(c) Let $x \in H^{7}\left(j ; \mathbb{F}_{2}\right)$ be the class that maps to $\Sigma^{7}(1)$ in the $A$-module extension of Lemma [7.10)(c). Then $\psi(x)=x \otimes 1+1 \otimes x$ since $H^{*}\left(j ; \mathbb{F}_{2}\right)=0$ for $0<*<7$, so $E(x)=\mathbb{F}_{2}\{1, x\}$ is a sub-coalgebra of $H^{*}\left(j ; \mathbb{F}_{2}\right)$ and there is a surjective composite $A$-module coalgebra homomorphism

$$
A \otimes E(x) \rightarrow A \otimes H^{*}\left(j ; \mathbb{F}_{2}\right) \rightarrow H^{*}\left(j ; \mathbb{F}_{2}\right) .
$$

Dually, let $b \in H_{7}\left(j ; \mathbb{F}_{2}\right)$ be the image of $\Sigma^{7}(1)$ in the $A_{*}$-comodule extension

$$
0 \rightarrow A_{*} \square_{A_{2 *}} \Sigma^{7} K_{*} \rightarrow H_{*}\left(j ; \mathbb{F}_{2}\right) \stackrel{\kappa}{\longrightarrow}\left(A / / A_{2}\right)_{*} \rightarrow 0 .
$$

Here $K_{*} \subset A_{2 *}$ has rank 17 , and contains $1 \in K_{*} \subset A_{2 *}$ in degree 0 . The dual of the surjection above is an injective $A_{*}$-comodule algebra homomorphism

$$
H_{*}\left(j ; \mathbb{F}_{2}\right) \rightarrow A_{*} \otimes E(b)
$$


that may otherwise be described as the composite of the $A_{*}$-comodule coaction $H_{*}\left(j ; \mathbb{F}_{2}\right) \rightarrow A_{*} \otimes H_{*}\left(j ; \mathbb{F}_{2}\right)$ and the algebra surjection $H_{*}\left(j ; \mathbb{F}_{2}\right) \rightarrow E(b)$.

We obtain a vertical map of $A_{*}$-comodule extensions

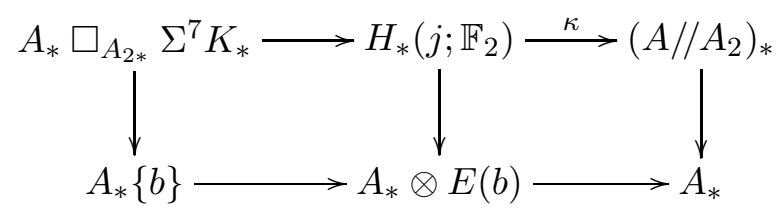

where the right hand square consists of $A_{*}$-comodule algebra homomorphisms, and the vertical maps are injective. At the left hand side we find the composite map

$$
A_{*} \square_{A_{2 *}} \Sigma^{7} K_{*} \rightarrow A_{*} \square_{A_{2 *}} \Sigma^{7} A_{2 *} \cong \Sigma^{7} A_{*} \cong A_{*}\{b\}
$$

that is dual to the surjection $A\{x\} \cong \Sigma^{7} A \rightarrow A \otimes_{A_{2}} \Sigma^{7} K$.

In the lower row the ideal $A_{*}\{b\}$ has square zero, since $b^{2}=0$, so also the ideal $\operatorname{ker}(\kappa)=A_{*} \square_{A_{2 *}} \Sigma^{7} K_{*}$ is a square-zero ideal CE56, XIV.2] in $H_{*}\left(j ; \mathbb{F}_{2}\right)$. Its module action by $H_{*}\left(j ; \mathbb{F}_{2}\right)$ therefore descends to one by $\left(A / / A_{2}\right)_{*}$. It can be described in terms of the algebra product $\phi$ on $A_{*}$ by the following commutative diagram with injective vertical maps:

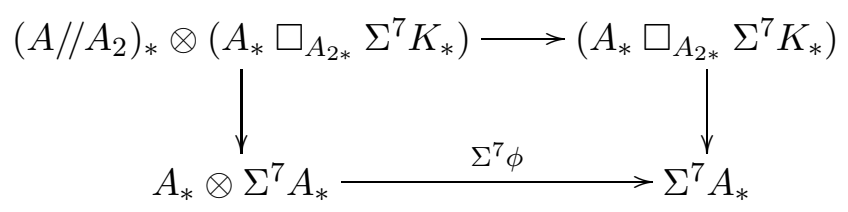

The proof is easy given the algebra embedding of $H_{*}\left(j ; \mathbb{F}_{2}\right)$ into $A_{*} \otimes E(b)$.

In fact, the square-zero ideal $A_{*} \square_{A_{2 *}} \Sigma^{7} K_{*}$ is a free $\left(A / / A_{2}\right)_{*}$-module of rank 17. For $A_{2} \subset A$ is a direct summand as an $A_{2}$-module, so dually $A_{*} \rightarrow A_{2 *}$ admits an $A_{2 *}$-comodule section $s: A_{2 *} \rightarrow A_{*}$. For example, the image of $s$ may be $\mathbb{F}_{2}\left\{\bar{\xi}_{1}^{i} \bar{\xi}_{2}^{j} \bar{\xi}_{3}^{k} \mid i<8, j<4, k<2\right\} \subset A_{*}$. We then have a map

$$
s \square i d: \Sigma^{7} K_{*} \cong A_{2 *} \square_{A_{2 *}} \Sigma^{7} K_{*} \rightarrow A_{*} \square_{A_{2 *}} \Sigma^{7} K_{*} .
$$

Its composite with the inclusion $A_{*} \square_{A_{2 *}} \Sigma^{7} K_{*} \rightarrow A_{*} \square_{A_{2 *}} \Sigma^{7} A_{2 *} \cong \Sigma^{7} A_{*}$ factors as the two inclusions $\Sigma^{7} K_{*} \rightarrow \Sigma^{7} A_{2 *} \rightarrow \Sigma^{7} A_{*}$.

Combining $s \square i d$ with the $\left(A / / A_{2}\right)_{*}$-module action on $A_{*} \square_{A_{2 *}} \Sigma^{7} K_{*}$ we obtain the left hand map $f$ in a commuting diagram

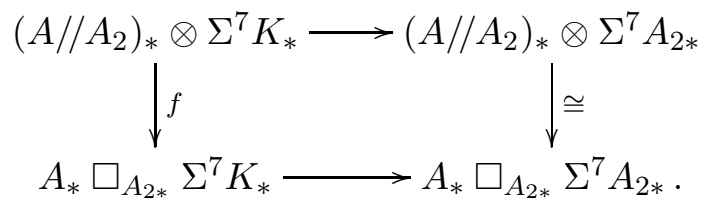


Here $A_{*} \square_{A_{2 *}} \Sigma^{7} A_{2 *} \cong \Sigma^{7} A_{*}$ and the right hand isomorphism exhibits $\Sigma^{7} A_{*}$ as a free $\left(A / / A_{2}\right)_{*}$-module on the generators given by the section $s: \Sigma^{7} A_{2 *} \rightarrow \Sigma^{7} A_{*}$. (It is a case of the Milnor-Moore comodule algebra theorem [MiMo65, 4.7].) The upper map is injective, hence so is

$$
f:\left(A / / A_{2}\right)_{*} \otimes \Sigma^{7} K_{*} \rightarrow A_{*} \square_{A_{2 *}} \Sigma^{7} K_{*} .
$$

But both sides have the same, finite dimension over $\mathbb{F}_{2}$ in each degree, so in fact $f$ is an isomorphism of $\left(A / / A_{2}\right)_{*}$-modules.

The fact that $\kappa$ splits as an algebra homomorphism is clear since $\left(A / / A_{2}\right)_{*}$ is a free graded commutative algebra over $\mathbb{F}_{2}$. However, the splitting is not an $A_{*}$ comodule homomorphism, and the $\left(A / / A_{2}\right)_{*}$-module isomorphism $f$ is not an $A_{*}$-comodule isomorphism. The $A_{*}$-comodule algebra structure on $H_{*}\left(j ; \mathbb{F}_{2}\right)$ may, if desired, be obtained by describing the image of the algebra generators under the $A_{*}$-comodule algebra embedding $H_{*}\left(j ; \mathbb{F}_{2}\right) \rightarrow A_{*} \otimes E(b)$.

Proposition 7.13 (a) For $p=2$ the Bökstedt spectral sequence $E_{* *}^{r}(j u)$ collapses at the $E^{2}$-term, with

$$
E_{* *}^{\infty}(j u) \cong H_{*}\left(j u ; \mathbb{F}_{2}\right) \otimes E\left(\sigma \bar{\xi}_{1}^{4}, \sigma \bar{\xi}_{2}^{2}, \sigma \bar{\xi}_{k} \mid k \geq 3\right) \otimes \Gamma(\sigma b) .
$$

(b) For $p$ odd the Bökstedt spectral sequence $E_{* *}^{r}(j u)$ collapses at the $E^{p}$ term, with

$$
E_{* *}^{\infty}(j u) \cong H_{*}\left(j u ; \mathbb{F}_{p}\right) \otimes E\left(\sigma \tilde{\xi}_{1}^{p}, \sigma \tilde{\xi}_{2}\right) \otimes P_{p}\left(\sigma \tilde{\tau}_{k} \mid k \geq 2\right) \otimes \Gamma(\sigma b) .
$$

(c) The Bökstedt spectral sequence for $j$ at $p=2$ has $E^{2}$-term

$$
E_{* *}^{2}(j) \cong H H_{*}\left(\left(A / / A_{2}\right)_{*}\right) \otimes H H_{*}\left(\mathbb{F}_{2} \oplus \Sigma^{7} K_{*}\right)
$$

where

$$
H H_{*}\left(\left(A / / A_{2}\right)_{*}\right) \cong\left(A / / A_{2}\right)_{*} \otimes E\left(\sigma \bar{\xi}_{1}^{8}, \sigma \bar{\xi}_{2}^{4}, \sigma \bar{\xi}_{3}^{2}, \sigma \bar{\xi}_{k} \mid k \geq 4\right)
$$

and

$$
H H_{q}\left(\mathbb{F}_{2} \oplus \Sigma^{7} K_{*}\right) \cong\left[\left(\Sigma^{7} K_{*}\right)^{\otimes q}\right]^{C_{q}} \oplus\left[\left(\Sigma^{7} K_{*}\right)^{\otimes q+1}\right]_{C_{q+1}} .
$$

In particular, $E_{* *}^{2}(j)$ is not flat as a module over $H_{*}\left(j ; \mathbb{F}_{2}\right)$.

Proof (a) The Bökstedt spectral sequence for $j u$ at $p=2$ begins

$$
E_{* *}^{2}(j u)=H_{*}\left(j u ; \mathbb{F}_{2}\right) \otimes E\left(\sigma \bar{\xi}_{1}^{4}, \sigma \bar{\xi}_{2}^{2}, \sigma \bar{\xi}_{k} \mid k \geq 3\right) \otimes \Gamma(\sigma b) .
$$

Proposition 4.8 applies, so a shortest non-zero differential must map from an algebra indecomposable to a coalgebra primitive and $A_{*}$-comodule primitive. 
Here we are referring to the $A_{*}$-comodule $H_{*}\left(j u ; \mathbb{F}_{2}\right)$-Hopf algebra structure on $E_{* *}^{2}(j u)$. The only possible algebra indecomposables are the $\gamma_{2^{k}}(\sigma b)$ in degrees $2^{k+2}$, for $k \geq 2$. The coalgebra primitives are $H_{*}\left(j u ; \mathbb{F}_{2}\right)\left\{\sigma b, \sigma \bar{\xi}_{1}^{4}, \sigma \bar{\xi}_{2}^{2}, \sigma \bar{\xi}_{k} \mid\right.$ $k \geq 3\}$, all in filtration $s=1$. A calculation with $\nu\left(\sigma \bar{\xi}_{2}^{2}\right)$ and $\nu\left(\sigma \bar{\xi}_{3}\right)$ shows that among these, the $A_{*}$-comodule primitives are equal to

$$
E(b) \otimes \mathbb{F}_{2}\left\{\sigma b, \sigma \bar{\xi}_{1}^{4}, \sigma \bar{\xi}_{k} \mid k \geq 4\right\} .
$$

These live in degrees $4,5,7,8,2^{k}$ and $2^{k}+3$, for $k \geq 4$. The image of a differential on $\gamma_{2^{k}}(\sigma b)$ must be in total degree $2^{k+2}-1$, for $k \geq 2$, but these degrees do not contain any simultaneous coalgebra- and comodule primitives. Therefore there are no non-zero differentials, and the spectral sequence collapses at the $E^{2}$-term.

(b) For $p$ odd the spectral sequence begins

$$
E_{* *}^{2}(j u)=H_{*}\left(j u ; \mathbb{F}_{p}\right) \otimes E\left(\sigma \tilde{\xi}_{1}^{p}, \sigma \tilde{\xi}_{k} \mid k \geq 2\right) \otimes \Gamma\left(\sigma b, \sigma \tilde{\tau}_{k} \mid k \geq 2\right) .
$$

By Proposition 5.6 we have $E^{2}=E^{p-1}$ and there are differentials

$$
d^{p-1}\left(\gamma_{p}\left(\sigma \tilde{\tau}_{k}\right)\right)=\sigma \tilde{\xi}_{k+1}
$$

for $k \geq 2$. This uses the relation $\beta Q^{p^{k}}\left(\tilde{\tau}_{k}\right)=\beta\left(\tilde{\tau}_{k+1}\right)=\tilde{\xi}_{k+1}$. There is also a potential differential

$$
d^{p-1}\left(\gamma_{p}(\sigma b)\right)=\sigma\left(\beta Q^{p q / 2}(b)\right),
$$

but $\beta Q^{p q / 2}(b)$ is in degree $p^{2} q-2$ of $H_{*}\left(j u ; \mathbb{F}_{p}\right)$, which is a trivial group, so this differential is zero. Hence

$$
E_{* *}^{p}(j u)=H_{*}\left(j u ; \mathbb{F}_{p}\right) \otimes E\left(\sigma \tilde{\xi}_{1}^{p}, \sigma \tilde{\xi}_{2}\right) \otimes P_{p}\left(\sigma \tilde{\tau}_{k} \mid k \geq 2\right) \otimes \Gamma(\sigma b) .
$$

Proposition 4.8 applies again, so a shortest differential must map from one of the algebra indecomposables $\gamma_{p^{k}}(\sigma b)$ in degrees $p^{k+1} q$, for $k \geq 2$. Its target must be among the coalgebra primitives, which are $H_{*}\left(j u ; \mathbb{F}_{p}\right)\left\{\sigma b, \sigma \tilde{\xi}_{1}^{p}, \sigma \tilde{\xi}_{2}, \sigma \tilde{\tau}_{k} \mid k \geq\right.$ $2\}$, all in filtration $s=1$. The target must also be $A_{*}$-comodule primitive. The formulas for the $A_{*}$-comodule structure on $H_{*}\left(j u ; \mathbb{F}_{p}\right)$ imply the following formulas:

$$
\begin{aligned}
\nu(\sigma b) & =1 \otimes \sigma b \\
\nu\left(\sigma \tilde{\xi}_{1}^{p}\right) & =1 \otimes \sigma \tilde{\xi}_{1}^{p}-\tau_{0} \otimes \sigma b \\
\nu\left(\sigma \tilde{\xi}_{2}\right) & =1 \otimes \sigma \tilde{\xi}_{2}+\bar{\xi}_{1} \otimes \sigma \tilde{\xi}_{1}^{p}+\tau_{1} \otimes \sigma b \\
\nu\left(\sigma \tilde{\tau}_{2}\right) & =1 \otimes \sigma \tilde{\tau}_{2}+\bar{\tau}_{0} \otimes \sigma \tilde{\xi}_{2}+\bar{\tau}_{1} \otimes \sigma \tilde{\xi}_{1}^{p}-\tau_{0} \tau_{1} \otimes \sigma b .
\end{aligned}
$$

The $\sigma \tilde{\tau}_{k}$ are $A_{*}$-comodule primitives for $k \geq 3$, in view of the relations $\left(\sigma \tilde{\tau}_{k}\right)^{p}=$ $\sigma \tilde{\tau}_{k+1}$ for $k \geq 2$, the formula for $\nu\left(\sigma \tilde{\tau}_{2}\right)$ and the fact that $\bar{\tau}_{0}, \bar{\tau}_{1}$ and $\tau_{0} \tau_{1}$ all 
square to zero. A calculation then shows that the simultaneous coalgebra- and comodule primitives are equal to

$$
E(b) \otimes \mathbb{F}_{p}\left\{\sigma b, \sigma \tilde{\tau}_{k} \mid k \geq 3\right\} .
$$

These live in degrees $p q, 2 p q-1,2 p^{k}$ and $2 p^{k}+p q-1$ for $k \geq 3$. The image of a differential $d^{r}\left(\gamma_{p^{k}}(\sigma b)\right)$ is in degree $p^{k+1} q-1$ for $k \geq 2$, which contains none of the possible target classes. Hence there are no further differentials, and the spectral sequence collapses at the $E^{p}$-term.

(c) By the Künneth formula

$$
E_{* *}^{2}(j)=H H_{*}\left(H_{*}\left(j ; \mathbb{F}_{2}\right)\right) \cong H H_{*}\left(\left(A / / A_{2}\right)_{*}\right) \otimes H H_{*}\left(\mathbb{F}_{2} \oplus \Sigma^{7} K_{*}\right) .
$$

Here the first tensor factor was identified in the discussion of tmf in Section 6 . By the following Lemma 7.14

$$
H H_{q}\left(\mathbb{F}_{2} \oplus \Sigma^{7} K_{*}\right) \cong\left[\Sigma^{7} K_{*}^{\otimes q}\right]^{C_{q}} \oplus\left[\Sigma^{7} K_{*}^{\otimes(q+1)}\right]_{C_{q+1}},
$$

and e.g. $H H_{1}\left(\mathbb{F}_{2} \oplus \Sigma^{7} K_{*}\right)$ is not flat as an $\mathbb{F}_{2} \oplus \Sigma^{7} K_{*}$-module.

Lemma 7.14 Let $k$ be a field and $V$ a graded $k$-vector space. The Hochschild homology of the split square-zero extension $k \oplus V$, with unit $(1,0)$ and multiplication $\left(k_{1}, v_{1}\right) \cdot\left(k_{2}, v_{2}\right)=\left(k_{1} k_{2}, k_{1} v_{2}+k_{2} v_{1}\right)$, is

$$
H H_{q}(k \oplus V) \cong\left[V^{\otimes q}\right]^{C_{q}} \oplus\left[V^{\otimes(q+1)}\right]_{C_{q+1}}
$$

where $\left[V^{\otimes q}\right]^{C_{q}} \subset V^{\otimes q}$ denotes the invariants of the cyclic group $C_{q}$ of order $q$ acting by cyclic permutations on $V^{\otimes q}$, and $\left[V^{\otimes q}\right]_{C_{q}}$ denotes the coinvariants of this action. When $\operatorname{dim}_{k} V \geq 2$ the Hochschild homology $H H_{*}(k \oplus V)$ is not flat as a module over $k \oplus V$.

Proof We compute $H H_{*}(k \oplus V)$ as the homology of the normalized Hochschild complex $N C_{*}(k \oplus V)$ with

$$
N C_{q}(k \oplus V)=(k \oplus V) \otimes V^{\otimes q} \cong V^{\otimes q} \oplus V^{\otimes(q+1)} .
$$

Here we remind the reader that the normalized Hochschild complex of a $k$ algebra $\Lambda$ is given by $N C_{q}(\Lambda)=\Lambda \otimes(\Lambda / k)^{\otimes q}$. We are considering the case $\Lambda=k \oplus V$, with $\Lambda / k=V$.

Since $V$ is a square-zero ideal, the Hochschild boundary $\partial$ is the direct sum over $q \geq 1$ of the operators

$$
1+(-1)^{q} t_{q}: V^{\otimes q} \rightarrow V^{\otimes q},
$$

i.e., $1+(-1)^{q} t_{q}$ on the $V^{\otimes q}$-summand and zero on the $V^{\otimes(q+1)}$-summand, where $t_{q}\left(v_{1} \otimes \cdots \otimes v_{q}\right)=(-1)^{\epsilon} v_{q} \otimes v_{1} \otimes \cdots \otimes v_{q-1}$ with $\epsilon=\left|v_{q}\right|\left(\left|v_{1}\right|+\cdots+\left|v_{q-1}\right|\right)$. 
Let the generator $T \in C_{q}$ act on $V^{\otimes q}$ as $(-1)^{q+1} t_{q}$, so $\partial$ is the sum of the operators $1-T$ (and $T^{q}$ acts as the identity). Clearly, then, the Hochschild homology is the direct sum over $q \geq 1$ of the kernels

$$
\operatorname{ker}(1-T)=\left[V^{\otimes q}\right]^{C_{q}}
$$

in degree $q$, and the cokernels

$$
\operatorname{cok}(1-T)=\left[V^{\otimes q}\right]_{C_{q}}
$$

in degree $(q-1)$, plus the term $k=V^{\otimes 0}$ in degree 0 .

For an example of the failure of flatness, let $V=k\{x, y\}$ with $x, y$ in odd degrees and $q=1$. Then $H H_{1}(k \oplus V) \cong V \oplus V_{C_{2}}^{\otimes 2} \cong k\{\sigma x, \sigma y, x \sigma x, x \sigma y \equiv y \sigma x, y \sigma y\}$ is not flat over $k \oplus V$.

Theorem 7.15 (a) For $p=2$ there is an isomorphism

$$
H_{*}\left(T H H(j u) ; \mathbb{F}_{2}\right) \cong H_{*}\left(j u ; \mathbb{F}_{2}\right) \otimes E\left(\sigma \bar{\xi}_{1}^{4}, \sigma \bar{\xi}_{2}^{2}\right) \otimes P\left(\sigma \bar{\xi}_{3}\right) \otimes \Gamma(\sigma b)
$$

of $H_{*}\left(j u ; \mathbb{F}_{2}\right)$-Hopf algebras.

(b) For $p$ odd there is an isomorphism

$$
H_{*}\left(T H H(j u) ; \mathbb{F}_{p}\right) \cong H_{*}\left(j u ; \mathbb{F}_{p}\right) \otimes E\left(\sigma \tilde{\xi}_{1}^{p}, \sigma \tilde{\xi}_{2}\right) \otimes P\left(\sigma \tilde{\tau}_{2}\right) \otimes \Gamma(\sigma b)
$$

of $H_{*}\left(j u ; \mathbb{F}_{p}\right)$-Hopf algebras.

Proof (a) In view of Proposition 7.13(a) we must identify the possible algebra extensions between the $E^{\infty}$-term

$$
E_{* *}^{\infty}(j u) \cong H_{*}\left(j u ; \mathbb{F}_{2}\right) \otimes E\left(\sigma \bar{\xi}_{1}^{4}, \sigma \bar{\xi}_{2}^{2}, \sigma \bar{\xi}_{k} \mid k \geq 3\right) \otimes \Gamma(\sigma b)
$$

and the abutment $H_{*}\left(T H H(j u) ; \mathbb{F}_{2}\right)$. Here $H_{*}\left(j u ; \mathbb{F}_{2}\right) \cong\left(A / / A_{1}\right)_{*} \otimes E(b)$ by Proposition 7.12

In $H_{*}\left(T H H(j u) ; \mathbb{F}_{2}\right)$ we have $(\sigma b)^{2}=Q^{4}(\sigma b)=\sigma Q^{4}(b)=0,\left(\sigma \bar{\xi}_{1}^{4}\right)^{2}=0$, $\left(\sigma \bar{\xi}_{2}^{2}\right)^{2}=0$ and $\left(\sigma \bar{\xi}_{k}\right)^{2}=\sigma \bar{\xi}_{k+1}$ for all $k \geq 3$, by Proposition [5.9] and the statement about Dyer-Lashof operations in Proposition 7.12(a). It remains to prove that we can find classes

$$
\gamma_{2^{k}} \in H_{4 \cdot 2^{k}}\left(T H H(j u) ; \mathbb{F}_{2}\right)
$$

that are represented by $\gamma_{2^{k}}(\sigma b)$ in $E_{* *}^{\infty}(j u)$ and satisfy $\gamma_{2^{k}}^{2}=0$, for all $k \geq 0$. We have just seen that we can take $\gamma_{1}=\sigma b$. So fix a number $k \geq 1$, and assume inductively that we have chosen classes $\gamma_{2^{m}}$ for $0 \leq m<k$ that are represented by $\gamma_{2^{m}}(\sigma b)$ and satisfy $\gamma_{2^{m}}^{2}=0$. 
We shall prove below that a class $\gamma_{2^{k}}$ representing $\gamma_{2^{k}}(\sigma b)$ can be chosen so that its square $\gamma_{2^{k}}^{2}$ is both an $H_{*}\left(j u ; \mathbb{F}_{2}\right)$-coalgebra primitive and an $A_{*}$-comodule primitive. We saw in the proof of Proposition [7.13)(a) that the simultaneous coalgebra- and comodule primitives are

$$
E(b) \otimes \mathbb{F}_{2}\left\{\sigma b, \sigma \bar{\xi}_{1}^{4}, \sigma \bar{\xi}_{m} \mid m \geq 4\right\} .
$$

When $k \geq 1$, the only such class in the degree of $\gamma_{2^{k}}^{2}$ is $\sigma \bar{\xi}_{k+3}=\left(\sigma \bar{\xi}_{k+2}\right)^{2}$. So either $\gamma_{2^{k}}^{2}=0$ or $\gamma_{2^{k}}^{2}=\left(\sigma \bar{\xi}_{k+2}\right)^{2}$. In the latter case we change $\gamma_{2^{k}}$ by subtracting $\sigma \bar{\xi}_{k+2}$, which does not alter the representative at the $E^{\infty}$-term. Thereby we have achieved $\gamma_{2^{k}}^{2}=0$, which will complete the inductive step.

To show that $\gamma_{2^{k}}^{2}$ can be arranged to be a coalgebra- and comodule primitive, we make use of the maps of $E^{\infty}$-terms and abutments induced by the commutative $S$-algebra homomorphism $\kappa: j u \rightarrow k u_{(2)}$. The target $E^{\infty}$-term is

$$
E_{* *}^{\infty}(k u) \cong H_{*}\left(k u ; \mathbb{F}_{2}\right) \otimes E\left(\sigma \bar{\xi}_{1}^{2}, \sigma \bar{\xi}_{2}^{2}, \sigma \bar{\xi}_{k} \mid k \geq 3\right)
$$

with abutment

$$
H_{*}\left(T H H(k u) ; \mathbb{F}_{2}\right) \cong H_{*}\left(k u ; \mathbb{F}_{2}\right) \otimes E\left(\sigma \bar{\xi}_{1}^{2}, \sigma \bar{\xi}_{2}^{2}\right) \otimes P\left(\sigma \bar{\xi}_{3}\right) .
$$

Here $H_{*}\left(k u ; \mathbb{F}_{2}\right) \cong\left(A / / E_{1}\right)_{*}$. Note that in degrees less than that of $\gamma_{2^{k}}, \kappa$ maps $H_{*}\left(T H H(j u) ; \mathbb{F}_{2}\right)$ modulo classes that square to zero, which by the inductive hypothesis is $\left(A / / A_{1}\right)_{*} \otimes P\left(\sigma \bar{\xi}_{3}\right)$, injectively into $H_{*}\left(T H H(k u) ; \mathbb{F}_{2}\right)$ modulo classes that square to zero, which is $\left(A / / E_{1}\right)_{*} \otimes P\left(\sigma \bar{\xi}_{3}\right)$. We shall refer to this property as the "near-injectivity of $\kappa$ ".

So choose any class $\gamma_{2^{k}}$ in $H_{*}\left(T H H(j u) ; \mathbb{F}_{2}\right)$ that is represented by $\gamma_{2^{k}}(\sigma b)$ in $E_{* *}^{\infty}(j u)$. We shall arrange that its image $\kappa \gamma_{2^{k}}$ in $H_{*}\left(T H H(k u) ; \mathbb{F}_{2}\right)$ squares to zero. If not, we can write

$$
\kappa \gamma_{2^{k}} \equiv c \cdot\left(\sigma \bar{\xi}_{3}\right)^{\ell}
$$

with $c \in\left(A / / E_{1}\right)_{*}$ not equal to zero, modulo classes in $H_{*}\left(T H H(k u) ; \mathbb{F}_{2}\right)$ that square to zero, and modulo similar terms with $c$ of lower degree (or equivalently, with higher exponent $\ell$ ). So $c \cdot\left(\sigma \bar{\xi}_{3}\right)^{\ell}$ is the "leading term" of $\kappa \gamma_{2^{k}}$ in $H_{*}\left(T H H(k u) ; \mathbb{F}_{2}\right)$ modulo classes that square to zero.

We divide into three cases. First, if $\ell=0$ and $\kappa \gamma_{2^{k}} \equiv c$ with $c \in\left(A / / E_{1}\right)_{*}$, we can apply the Hopf algebra counit $\epsilon: \operatorname{THH}(R) \rightarrow R$ to see that $c=\epsilon\left(\kappa \gamma_{2^{k}}\right)=$ $\kappa\left(\epsilon \gamma_{2^{k}}\right)$ is in the image of $\kappa: H_{*}\left(j u ; \mathbb{F}_{2}\right) \rightarrow H_{*}\left(k u ; \mathbb{F}_{2}\right)$, so that in fact $c \in$ $\left(A / / A_{1}\right)_{*}$. We can then replace $\gamma_{2^{k}}$ by $\gamma_{2^{k}}-c$ without altering its representative in $E^{\infty}$, and thus eliminate the case $\ell=0$.

Second, if $\ell=1$ and $\kappa \gamma_{2^{k}} \equiv c \cdot \sigma \bar{\xi}_{3}$ with $c \in\left(A / / E_{1}\right)_{*}$, we need to argue that $c \in\left(A / / A_{1}\right)_{*}$. If not, we can write $c=\bar{\xi}_{1}^{2} \cdot d$ with $d \in\left(A / / A_{1}\right)_{*}$. Here 
$\nu(c)=\psi(c) \equiv c \otimes 1+d \otimes \bar{\xi}_{1}^{2}$ modulo terms with first tensor factor in degree less than $|d|$. Note from the structure of $H_{*}\left(T H H(k u) ; \mathbb{F}_{2}\right)$ that $\kappa \gamma_{2^{k}} \equiv c \cdot \sigma \bar{\xi}_{3}$ modulo classes that square to zero, and similar terms $e \cdot\left(\sigma \bar{\xi}_{3}\right)^{\ell}$ with $\ell \geq 2$, so $|e|<|d|$. So $\nu\left(\kappa \gamma_{2^{k}}\right) \equiv c \otimes \sigma \bar{\xi}_{3}+d \otimes \bar{\xi}_{1}^{2} \sigma \bar{\xi}_{3}$ modulo classes that square to zero, and terms with first factor in lower degree. This must be the image of $\nu\left(\gamma_{2^{k}}\right)$ under $1 \otimes \kappa$, so

$$
\nu\left(\gamma_{2^{k}}\right) \equiv c \otimes \sigma \bar{\xi}_{3}+d \otimes x
$$

for some class $x \in H_{*}\left(T H H(j u) ; \mathbb{F}_{2}\right)$ with $\kappa(x) \equiv \bar{\xi}_{1}^{2} \sigma \bar{\xi}_{3}$. But there exists no such class $x$, since $H_{*}\left(T H H(j u) ; \mathbb{F}_{2}\right)$ modulo classes that square to zero is trivial in degree 10. (This is clear by inspection of the Bökstedt spectral sequence, where the group that could cause a problem, $E_{2,8}^{2}(j u)$, is in fact zero.) This contradiction shows that $c \in\left(A / / A_{1}\right)_{*}$, and we can alter $\gamma_{2^{k}}$ by $c \cdot \sigma \bar{\xi}_{3}$ without altering the representative at $E^{\infty}$, and thus eliminate the case $\ell=1$.

Third, for the remaining cases $\ell \geq 2$ we consider the coalgebra coproduct. We find

$$
\psi\left(\kappa \gamma_{2^{k}}\right) \equiv \sum_{i+j=\ell} c \cdot\left(\sigma \bar{\xi}_{3}\right)^{i} \otimes\left(\sigma \bar{\xi}_{3}\right)^{j}
$$

in $H_{*}\left(T H H(k u) ; \mathbb{F}_{2}\right) \otimes_{H_{*}\left(k u ; \mathbb{F}_{2}\right)} H_{*}\left(T H H(k u) ; \mathbb{F}_{2}\right)$, modulo classes that square to zero and similar terms with $c$ of lower degree. Since $\ell \geq 2$ this sum includes some terms $c \cdot\left(\sigma \bar{\xi}_{3}\right)^{i} \otimes\left(\sigma \bar{\xi}_{3}\right)^{j}$ with both $i$ and $j$ positive, so that $c \cdot\left(\sigma \bar{\xi}_{3}\right)^{i}$ and $\left(\sigma \bar{\xi}_{3}\right)^{j}$ are both in degree less than that of $\gamma_{2^{k}}$. This is the image under $\kappa \otimes \kappa$ of $\psi\left(\gamma_{2^{k}}\right)$ in $H_{*}\left(T H H(j u) ; \mathbb{F}_{2}\right) \otimes_{H_{*}\left(j u ; \mathbb{F}_{2}\right)} H_{*}\left(T H H(j u) ; \mathbb{F}_{2}\right)$, so it follows by the near-injectivity of $\kappa$ that $c$ in fact lies in $\left(A / / A_{1}\right)_{*} \subset\left(A / / E_{1}\right)_{*}$.

Then we can change the chosen $\gamma_{2^{k}}$ in $H_{*}\left(T H H(j u) ; \mathbb{F}_{2}\right)$ by subtracting $c \cdot\left(\sigma \bar{\xi}_{3}\right)^{\ell}$ from it, and thus remove the "leading" term $c \cdot\left(\sigma \bar{\xi}_{3}\right)^{\ell}$ from $\kappa \gamma_{2^{k}}$. By repeating this process we can arrange that $\gamma_{2^{k}}$ has be chosen so that $\kappa \gamma_{2^{k}}$ is zero modulo classes that square to zero, i.e., that $\kappa \gamma_{2^{k}}^{2}=0$.

Then $\psi\left(\gamma_{2^{k}}\right) \equiv \gamma_{2^{k}} \otimes 1+1 \otimes \gamma_{2^{k}}$ modulo classes that square to zero. For $\psi\left(\kappa \gamma_{2^{k}}^{2}\right)=0$ so any other terms in $\psi\left(\gamma_{2^{k}}\right)$ must map under $\kappa$ to classes that square to zero, hence square to zero themselves by the near-injectivity of $\kappa$. Hence $\gamma_{2^{k}}^{2}$ is a coalgebra primitive.

Next consider the $A_{*}$-comodule coaction. If $\nu\left(\gamma_{2^{k}}\right) \equiv 1 \otimes \gamma_{2^{k}}$ modulo classes that square to zero, then $\nu\left(\gamma_{2^{k}}^{2}\right)=1 \otimes \gamma_{2^{k}}^{2}$ and $\gamma_{2^{k}}^{2}$ is an $A_{*}$-comodule primitive, as desired. Otherwise, we can write

$$
\nu\left(\gamma_{2^{k}}\right) \equiv a \otimes\left(\sigma \bar{\xi}_{3}\right)^{\ell}
$$


with $a \in A_{*}$ in positive degree, modulo classes in $A_{*} \otimes H_{*}\left(T H H(j u) ; \mathbb{F}_{2}\right)$ that square to zero, and modulo similar terms with $a$ of lower degree. Then $\nu\left(\gamma_{2^{k}}^{2}\right) \equiv$ $a^{2} \otimes\left(\sigma \bar{\xi}_{3}\right)^{2 \ell}$ modulo terms with $a$ of lower degree. Applying $\kappa$ yields $a^{2} \otimes$ $\left(\sigma \bar{\xi}_{3}\right)^{2 \ell}=0$, since $\kappa \gamma_{2^{k}}^{2}=0$, so $a^{2}=0$. This is impossible for $a \neq 0$, so we conclude that $\gamma_{2^{k}}^{2}$ is indeed an $A_{*}$-comodule primitive.

This completes the proof.

(b) Also in the odd primary case we must identify the algebra extensions between the $E^{\infty}$-term from Proposition 17.13(b)

$$
E_{* *}^{\infty}(j u)=H_{*}\left(j u ; \mathbb{F}_{p}\right) \otimes E\left(\sigma \tilde{\xi}_{1}^{p}, \sigma \tilde{\xi}_{2}\right) \otimes P_{p}\left(\sigma \tilde{\tau}_{k} \mid k \geq 2\right) \otimes \Gamma(\sigma b)
$$

and the abutment $H_{*}\left(T H H(j u) ; \mathbb{F}_{p}\right)$. In the latter we have $(\sigma b)^{p}=Q^{p q / 2}(\sigma b)=$ $\sigma Q^{p q / 2}(b)=0$ and $\left(\sigma \tilde{\tau}_{k}\right)^{p}=Q^{p^{k}}\left(\sigma \tilde{\tau}_{k}\right)=\sigma Q^{p^{k}}\left(\tilde{\tau}_{k}\right)=\sigma \tilde{\tau}_{k+1}$, by Propositions [5.9] and $\mathbf{7 . 1 2}(\mathrm{b})$. The classes $\sigma \tilde{\xi}_{1}^{p}$ and $\sigma \tilde{\xi}_{2}$ are in odd degree, and therefore have square zero, since $p$ is odd.

It remains to prove that we can find classes $\gamma_{p^{k}} \in H_{p^{k+1} q}\left(T H H(j u) ; \mathbb{F}_{p}\right)$ that are represented by $\gamma_{p^{k}}(\sigma b)$ in $E_{* *}^{\infty}(j u)$ and satisfy $\gamma_{p^{k}}^{p}=0$, for all $k \geq 0$. We have just verified this for $k=0$.

The remaining inductive proof follows exactly the same strategy as in the $p=2$ case. Instead of working modulo classes that square to zero we work modulo classes with $p$-th power equal to zero. The $S$-algebra map $\kappa: j u \rightarrow \ell$ induces an algebra homomorphism in homology that has the required "nearinjectivity" property, etc. In the tricky case when $\kappa \gamma_{p^{k}} \equiv c \cdot \sigma \tilde{\tau}_{2}$, modulo classes in $H_{*}\left(T H H(\ell) ; \mathbb{F}_{p}\right)$ that have trivial $p$-th power, and modulo similar terms with $c$ of lower degree, we have $c \in\left(A / / E_{1}\right)_{*}$ and must argue that in fact $c \in\left(A / / A_{1}\right)_{*}$. Writing $c=\bar{\xi}_{1}^{f} \cdot d$, with $d \in\left(A / / A_{1}\right)_{*}$ and $0 \leq f<p$ we assume $0<f<p$ and reach a contradiction. The coaction $\nu\left(\kappa \gamma_{p^{k}}\right)$ contains a term

$f \bar{\xi}_{1}^{f-1} d \otimes \bar{\xi}_{1} \sigma \bar{\tau}_{2}$, and we must check that there is no class $x \in H_{*}\left(T H H(j u) ; \mathbb{F}_{p}\right)$ with $\kappa x=\bar{\xi}_{1} \sigma \bar{\tau}_{2}$. This is again clear by the Bökstedt spectral sequence. The rest of the proof can safely be omitted.

\section{Topological $K$-theory revisited}

We now wish to pass from the homology of the spectra $T H H(k u)$ and $T H H(k o)$ to their homotopy. The first case is the analogue for $p=2$ of the discussion in [MS93, $\S \S 5-7]$, where McClure and Staffeldt compute the mod $p$ homotopy groups $\pi_{*}(T H H(\ell) ; \mathbb{Z} / p)$ of the Adams summand $\ell \subset k u_{(p)}$ for odd primes $p$. 
The idea is to compute homotopy groups using the Adams spectral sequence

$$
E_{2}^{s, t}=\operatorname{Ext}_{A_{*}}^{s, t}\left(\mathbb{F}_{p}, H_{*}\left(X ; \mathbb{F}_{p}\right)\right) \Longrightarrow \pi_{t-s}(X)_{p}^{\wedge},
$$

which is strongly convergent for bounded below spectra $X$ of $\mathbb{Z}_{p}$-finite type. This is difficult for $X=T H H(k u)$ or $T H H(k o)$ at $p=2$, just as for $T H H(\ell)$ at $p$ odd, but becomes manageable after introducing suitable finite coefficients, e.g. after smashing $T H H(k u)$ with the mod 2 Moore spectrum $M=C_{2}$, or smashing $T H H(k o)$ with the Mahowald spectrum $Y=C_{2} \wedge C_{\eta}$ Mah82. Here $C_{f}$ denotes the mapping cone of a map $f$. Neither of these finite CW spectra are ring spectra, so an extra argument is needed to have products on $T H H(k u) \wedge M$ and $T H H(k o) \wedge Y$. We shall manage with the following very weak version of a ring spectrum.

Definition 8.1 A $\mu$-spectrum (or an $A_{2}$ ring spectrum) is a spectrum $R$ with a unit $\eta: S \rightarrow R$ and multiplication $\mu: R \wedge R \rightarrow R$ that is left and right unital, but not necessarily associative or commutative.

The following result is similar to [0k79, 1.5], but slightly easier.

Lemma 8.2 Let $R$ be a $\mu$-spectrum and let

$$
S^{k} \stackrel{f}{\longrightarrow} S^{0} \stackrel{i}{\longrightarrow} C_{f} \stackrel{\pi}{\longrightarrow} S^{k+1}
$$

be a cofiber sequence such that $i d_{R} \wedge i d_{C_{f}} \wedge f: R \wedge \Sigma^{k} C_{f} \rightarrow R \wedge C_{f}$ is nullhomotopic. Then there exists a multiplication $\mu:\left(R \wedge C_{f}\right) \wedge\left(R \wedge C_{f}\right) \rightarrow\left(R \wedge C_{f}\right)$ that makes $R \wedge C_{f}$ a $\mu$-spectrum and $i d_{R} \wedge i: R \rightarrow R \wedge C_{f}$ a map of $\mu$-spectra.

Proof A choice of null-homotopy provides a splitting $m: R \wedge C_{f} \wedge C_{f} \rightarrow R \wedge C_{f}$ in the cofiber sequence

$$
R \wedge \Sigma^{k} C_{f} \stackrel{i d \wedge i d \wedge f}{\longrightarrow} R \wedge C_{f} \stackrel{i d \wedge i d \wedge i}{\longrightarrow} R \wedge C_{f} \wedge C_{f} \stackrel{i d \wedge i d \wedge \pi}{\longrightarrow} R \wedge \Sigma^{k+1} C_{f}
$$

which satisfies $m(i d \wedge i d \wedge i) \simeq i d$ (right unitality). The difference $i d-m(i d \wedge$ $i \wedge i d): R \wedge C_{f} \rightarrow R \wedge C_{f}$ restricts trivially over $i d \wedge i: R \rightarrow R \wedge C_{f}$, so extends over $i d \wedge \pi: R \wedge C_{f} \rightarrow R \wedge S^{k+1}$ to a map $\phi: R \wedge S^{k+1} \rightarrow R \wedge C_{f}$. There is a short exact sequence

$$
\left[R \wedge \Sigma^{k+1} C_{f}, R \wedge C_{f}\right] \stackrel{i^{*}}{\longrightarrow}\left[R \wedge S^{k+1}, R \wedge C_{f}\right] \stackrel{f^{*}}{\longrightarrow}\left[R \wedge S^{2 k+1}, R \wedge C_{f}\right]
$$

where the homomorphism $f^{*}=(i d \wedge f \wedge i d)^{*}$ is trivial, because it can be identified with $(i d \wedge i d \wedge f)^{*}:\left[R \wedge C_{f}, R\right] \rightarrow\left[R \wedge \Sigma^{k} C_{f}, R\right]$ under SpanierWhitehead duality, and we have assumed that this map $i d \wedge i d \wedge f$ is nullhomotopic. (The Spanier-Whitehead dual of $C_{f}$ is $\Sigma^{-(k+1)} C_{f}$.) Thus $i^{*}=$ 
$\left(i d \wedge \Sigma^{k+1} i\right)^{*}$ is surjective and $\phi$ extends further over $i d \wedge \Sigma^{k+1} i$ to a map $\psi: R \wedge \Sigma^{k+1} C_{f} \rightarrow R \wedge C_{f}$. Then we can subtract $\psi \circ(i d \wedge i d \wedge \pi)$ from $m$ so as to make $m(i d \wedge i \wedge i d) \simeq i d$ (left unitality), without destroying the right unitality. Once this is achieved, the required multiplication is defined as the composite

$$
\begin{aligned}
\left(R \wedge C_{f}\right) \wedge\left(R \wedge C_{f}\right) \stackrel{i d \wedge \tau \wedge i d}{\longrightarrow} R \wedge R \wedge C_{f} \wedge C_{f} \\
\left.\right|_{\mu \wedge i d \wedge i d} \\
R \wedge C_{f} \wedge C_{f} \stackrel{m}{\longrightarrow}\left(R \wedge C_{f}\right),
\end{aligned}
$$

where $\tau$ denotes the twist map.

In the case $f=2: S^{0} \rightarrow S^{0}$, the Moore spectrum $M=C_{2}$ has cohomology $H^{*}\left(M ; \mathbb{F}_{2}\right)=E\left(S q^{1}\right)$, which equals $E_{1} / / E\left(Q_{1}\right)$ as an $E_{1}$-module. By the Cartan formula $S q^{2}$ acts nontrivially on $H^{*}\left(M \wedge M ; \mathbb{F}_{2}\right)$, and therefore $M$ does not split off from $M \wedge M$. So $M$ is not a $\mu$-spectrum, and the map $2: M \rightarrow M$ is not null-homotopic. It must therefore factor as the composite

$$
M \stackrel{\pi}{\longrightarrow} S^{1} \stackrel{\eta}{\longrightarrow} S^{0} \stackrel{i}{\longrightarrow} M .
$$

See e.g. AT65, 1.1].

Similarly, in the case $f=\eta: S^{1} \rightarrow S^{0}$ the mapping cone $C_{\eta}$ has cohomology $H^{*}\left(C_{\eta} ; \mathbb{F}_{2}\right)=E\left(S q^{2}\right)$. By the Cartan formula $S q^{4}$ acts nontrivially on $H^{*}\left(C_{\eta} \wedge\right.$ $\left.C_{\eta} ; \mathbb{F}_{2}\right)$, and therefore $C_{\eta}$ does not split off from $C_{\eta} \wedge C_{\eta}$. In particular, the map $\eta: \Sigma C_{\eta} \rightarrow C_{\eta}$ is not null-homotopic, and must factor as the composite

$$
\Sigma C_{\eta} \stackrel{\pi}{\longrightarrow} S^{3} \stackrel{\nu}{\longrightarrow} S^{0} \stackrel{i}{\longrightarrow} C_{\eta} .
$$

Here $\nu \in \pi_{3}(S)$ is the Hopf invariant one class. The Mahowald spectrum $Y=$ $C_{2} \wedge C_{\eta}$ has cohomology $H^{*}\left(Y ; \mathbb{F}_{2}\right)=E\left(S q^{1}, S q^{2}\right)$, which equals $A_{1} / / E\left(Q_{1}\right)$ as an $A_{1}$-module.

Lemma 8.5 (a) $T H H(k u) \wedge M$ is a $\mu$-spectrum.

(b) $T H H(k o) \wedge C_{\eta}$ and $T H H(k o) \wedge Y$ are $\mu$-spectra.

Proof (a) Let $T=T H H(k u)$. By (8.3) the map $1 \wedge 2: T \wedge M \rightarrow T \wedge M$ factors through $1 \wedge \eta: \Sigma T \rightarrow T$, which in turn factors as

$$
\Sigma T \stackrel{1 \wedge \eta}{\longrightarrow} T \wedge k u \rightarrow T
$$


since $T=T H H(k u)$ is a $k u$-module spectrum. But $\eta \in \pi_{1}(S)$ maps to zero in $\pi_{1}(k u)$, so this map is null-homotopic, and Lemma 8.2 applies.

(b) Let $T=T H H(k o)$ and $R=T H H(k o) \wedge C_{\eta}$. By (8.4) the map $1 \wedge \eta: \Sigma R \rightarrow$ $R$ factors through $1 \wedge \nu: \Sigma^{3} T \rightarrow T$, which in turn factors as

$$
\Sigma^{3} T \stackrel{1 \wedge \nu}{\longrightarrow} T \wedge k o \rightarrow T,
$$

since $T=T H H(k o)$ is a $k o$-module spectrum. But $\nu \in \pi_{3}(S)$ maps to zero in $\pi_{3}(k o)$, so $1 \wedge \eta: \Sigma R \rightarrow R$ is null-homotopic, and Lemma 8.2 applies again to prove that $R$ is a $\mu$-spectrum under $T$.

Using once more that $1 \wedge \eta: \Sigma R \rightarrow R$ is null-homotopic we get that $1 \wedge 2: R \wedge$ $M \rightarrow R \wedge M$ is null-homotopic by (8.3), and so $R \wedge M=T H H(k o) \wedge Y$ is a $\mu$-spectrum under $R$.

Lemma 8.6 Let $B \subset A$ be a sub Hopf algebra and let $N$ be an $A_{*}$-comodule algebra. Then there is an isomorphism of $A_{*}$-comodule algebras

$$
(A / / B)_{*} \otimes N \cong A_{*} \square_{B_{*}} N .
$$

Here $B_{*}$ is the quotient Hopf algebra of $A_{*}$ dual to $B$, and $(A / / B)_{*}=A_{*} \square_{B_{*}} \mathbb{F}_{p}$ is dual to $A \otimes_{B} \mathbb{F}_{p}$.

Proof This is analogous to the usual $G$-homeomorphism $G / H \times X \cong G \times{ }_{H} X$ for a $G$-space $X$ and subgroup $H \subset G$. Let $i:(A / / B)_{*} \rightarrow A_{*}$ be the inclusion and $\nu: N \rightarrow A_{*} \otimes N$ the coaction. The composite homomorphism

$$
(A / / B)_{*} \otimes N \stackrel{i \otimes \nu}{\longrightarrow} A_{*} \otimes A_{*} \otimes N \stackrel{\phi \otimes 1}{\longrightarrow} A_{*} \otimes N
$$

equalizes the two maps to $A_{*} \otimes B_{*} \otimes N$, and hence factors uniquely through $A_{*} \square_{B_{*}} N$. An explicit inverse can be constructed using the Hopf algebra conjugation $\chi$ on $A_{*}$.

Recall the $n$-th connective Morava $K$-theory spectrum $k(n)$, with homotopy $\pi_{*} k(n)=\mathbb{F}_{p}\left[v_{n}\right]$ where $\left|v_{n}\right|=2\left(p^{n}-1\right)$, and cohomology $H^{*}\left(k(n) ; \mathbb{F}_{p}\right) \cong$ $A / / E\left(Q_{n}\right)$ [BM72]. Dually, $H_{*}\left(k(n) ; \mathbb{F}_{p}\right) \cong\left(A / / E\left(Q_{n}\right)\right)_{*} \subset A_{*}$. In particular, for $n=1$ and $p=2$ we have $k(1) \simeq k u \wedge M$ with $H_{*}\left(k(1) ; \mathbb{F}_{2}\right) \cong\left(A / / E\left(Q_{1}\right)\right)_{*} \cong$ $P\left(\bar{\xi}_{1}, \bar{\xi}_{2}^{2}, \bar{\xi}_{k} \mid k \geq 3\right)$. The $n$-th periodic Morava $K$-theory spectrum $K(n)$ is the telescope $v_{n}^{-1} k(n)$ of the iterated maps $v_{n}: k(n) \rightarrow \Sigma^{2\left(1-p^{n}\right)} k(n)$, with homotopy $\pi_{*} K(n)=\mathbb{F}_{p}\left[v_{n}, v_{n}^{-1}\right]$. These are (non-commutative) $S$-algebras for all $p$ and $n$ ([Rob89, [La03]). 
Proposition 8.7 (a) There are $A_{*}$-comodule algebra isomorphisms

$$
\begin{aligned}
H_{*}\left(T H H(k u) \wedge M ; \mathbb{F}_{2}\right) & \cong\left(A / / E\left(Q_{1}\right)\right)_{*} \otimes E\left(\sigma \bar{\xi}_{1}^{2}, \sigma \bar{\xi}_{2}^{2}\right) \otimes P\left(\sigma \bar{\xi}_{3}\right) \\
& \cong H_{*}\left(k(1) ; \mathbb{F}_{2}\right) \otimes E\left(\lambda_{1}, \lambda_{2}\right) \otimes P(\mu) .
\end{aligned}
$$

Here $\nu\left(\sigma \bar{\xi}_{1}^{2}\right)=1 \otimes \sigma \bar{\xi}_{1}^{2}, \nu\left(\sigma \bar{\xi}_{2}^{2}\right)=1 \otimes \sigma \bar{\xi}_{2}^{2}$, and $\nu\left(\sigma \bar{\xi}_{3}\right)=1 \otimes \sigma \bar{\xi}_{3}+\bar{\xi}_{1} \otimes \sigma \bar{\xi}_{2}^{2}$.

The classes $\lambda_{1}=\sigma \bar{\xi}_{1}^{2}, \lambda_{2}=\sigma \bar{\xi}_{2}^{2}$ and $\mu=\sigma \bar{\xi}_{3}+\bar{\xi}_{1} \cdot \sigma \bar{\xi}_{2}^{2}$ (in degrees 3, 7 and 8, respectively) are $A_{*}$-comodule primitives.

(b) There are $A_{*}$-comodule algebra isomorphisms

$$
\begin{aligned}
H_{*}\left(T H H(k o) \wedge Y ; \mathbb{F}_{2}\right) & \cong\left(A / / E\left(Q_{1}\right)\right)_{*} \otimes E\left(\sigma \bar{\xi}_{1}^{4}, \sigma \bar{\xi}_{2}^{2}\right) \otimes P\left(\sigma \bar{\xi}_{3}\right) \\
& \cong H_{*}\left(k(1) ; \mathbb{F}_{2}\right) \otimes E\left(\lambda_{1}, \lambda_{2}\right) \otimes P(\mu) .
\end{aligned}
$$

Here $\nu\left(\sigma \bar{\xi}_{1}^{4}\right)=1 \otimes \sigma \bar{\xi}_{1}^{4}, \nu\left(\sigma \bar{\xi}_{2}^{2}\right)=1 \otimes \sigma \bar{\xi}_{2}^{2}+\bar{\xi}_{1}^{2} \otimes \sigma \bar{\xi}_{1}^{4}$ and $\nu\left(\sigma \bar{\xi}_{3}\right)=1 \otimes \sigma \bar{\xi}_{3}+$ $\bar{\xi}_{1} \otimes \sigma \bar{\xi}_{2}^{2}+\bar{\xi}_{2} \otimes \sigma \bar{\xi}_{1}^{4}$.

The exterior classes $\lambda_{1}=\sigma \bar{\xi}_{1}^{4}$ and $\lambda_{2}=\sigma \bar{\xi}_{2}^{2}+\bar{\xi}_{1}^{2} \cdot \sigma \bar{\xi}_{1}^{4}$ are $A_{*}$-comodule primitives, while $\mu=\sigma \bar{\xi}_{3}+\bar{\xi}_{1} \cdot \sigma \bar{\xi}_{2}^{2}$ has

$$
\nu(\mu)=1 \otimes \mu+\bar{\xi}_{1}^{2} \otimes \bar{\xi}_{1} \cdot \lambda_{1}+\left(\bar{\xi}_{2}+\bar{\xi}_{1}^{3}\right) \otimes \lambda_{1} .
$$

The squared class $\mu^{2}=\left(\sigma \bar{\xi}_{3}\right)^{2}$ is $A_{*}$-comodule primitive.

In each case, the homology algebra on the left hand side has the unit and product induced by the $\mu$-spectrum structure from Lemma 8.5. This product is in fact associative and graded commutative, in view of the exhibited additive and multiplicative isomorphism with the associative and graded commutative algebra on the right hand side.

Proof (a) By Corollary 5.14(a) there is an $A_{*}$-comodule algebra isomorphism

$$
H_{*}\left(T H H(k u) \wedge M ; \mathbb{F}_{2}\right) \cong\left(A / / E_{1}\right)_{*} \otimes\left(E_{1} / / E\left(Q_{1}\right)\right)_{*} \otimes E\left(\sigma \bar{\xi}_{1}^{2}, \sigma \bar{\xi}_{2}^{2}\right) \otimes P\left(\sigma \bar{\xi}_{3}\right)
$$

with the diagonal $A_{*}$-comodule structure on the first two tensor factors, and the claimed coaction on the remaining generators.

Since $H_{*}\left(M ; \mathbb{F}_{2}\right) \cong\left(E_{1} / / E\left(Q_{1}\right)\right)_{*}$ is in fact an $A_{*}$-comodule algebra, there is an $A_{*}$-comodule algebra isomorphism

$$
\left(A / / E_{1}\right)_{*} \otimes\left(E_{1} / / E\left(Q_{1}\right)\right)_{*} \cong A_{*} \square_{E_{1 *}}\left(E_{1} / / E\left(Q_{1}\right)\right)_{*} \cong\left(A / / E\left(Q_{1}\right)\right)_{*}
$$

by Lemma 8.6. We are free to replace the polynomial generator $\sigma \bar{\xi}_{3}$ by the primitive class $\mu$, since their difference $\bar{\xi}_{1} \cdot \sigma \bar{\xi}_{2}^{2}$ has square zero.

(b) By Theorem 6.2 (a) there is an $A_{*}$-comodule algebra isomorphism

$$
H_{*}\left(T H H(k o) \wedge Y ; \mathbb{F}_{2}\right) \cong\left(A / / A_{1}\right)_{*} \otimes\left(A_{1} / / E\left(Q_{1}\right)\right)_{*} \otimes E\left(\sigma \bar{\xi}_{1}^{4}, \sigma \bar{\xi}_{2}^{2}\right) \otimes P\left(\sigma \bar{\xi}_{3}\right),
$$


with the claimed coaction on the exterior and polynomial generators. Again, by Lemma 8.6 there is an isomorphism of $A_{*}$-comodule algebras

$$
\left(A / / A_{1}\right)_{*} \otimes\left(A_{1} / / E\left(Q_{1}\right)\right)_{*} \cong A_{*} \square_{A_{1 *}}\left(A_{1} / / E\left(Q_{1}\right)\right)_{*} \cong\left(A / / E\left(Q_{1}\right)\right)_{*} .
$$

The classes $\lambda_{1}, \lambda_{2}$ and $\mu$ are defined as in the statement of the proposition, and their coactions are obtained by direct calculation.

Lemma 8.8 (a) The $E_{2}$-term of the Adams spectral sequence converging to $\pi_{*}(T H H(k u) \wedge M)$ is

$$
E_{2}^{* *} \cong P\left(v_{1}\right) \otimes E\left(\lambda_{1}, \lambda_{2}\right) \otimes P(\mu)
$$

with $\lambda_{1}, \lambda_{2}, \mu$ and $v_{1}=\left[\xi_{2}\right]$ in bidegrees $(0,5),(0,7),(0,8)$ and $(1,3)$, respectively.

(b) The $E_{2}$-term of the Adams spectral sequence for $\pi_{*}(T H H(k o) \wedge Y)$ is

$$
E_{2}^{* *} \cong\left(P\left(v_{1}\right) \otimes E\left(\lambda_{2}, \lambda_{3}\right) \oplus E\left(\lambda_{2}\right)\left\{\lambda_{1}\right\}\right) \otimes P\left(\mu^{2}\right)
$$

with $\lambda_{1}, \lambda_{2}, \lambda_{3}=\lambda_{1} \mu, \mu^{2}$ and $v_{1}=\left[\xi_{2}\right]$ in bidegrees $(0,5),(0,7),(0,13)$, $(0,16)$ and $(1,3)$, respectively. It is the homology of the algebra

$$
P\left(v_{1}\right) \otimes E\left(\lambda_{1}, \lambda_{2}\right) \otimes P(\mu)
$$

with respect to the differential $d(\mu)=v_{1} \lambda_{1}$, with cycles $\lambda_{1}, \lambda_{2}$ and $v_{1}$.

Proof (a) By change-of-rings, the $E_{2}$-term of the Adams spectral sequence for $X=T H H(k u) \wedge M$ is

$$
\begin{aligned}
E_{2}^{* *} & =\operatorname{Ext}_{A_{*}}^{* *}\left(\mathbb{F}_{2},\left(A / / E\left(Q_{1}\right)\right)_{*} \otimes E\left(\lambda_{1}, \lambda_{2}\right) \otimes P(\mu)\right) \\
& \cong \operatorname{Ext}_{E\left(Q_{1}\right)_{*}}^{* *}\left(\mathbb{F}_{2}, E\left(\lambda_{1}, \lambda_{2}\right) \otimes P(\mu)\right) \\
& \cong P\left(v_{1}\right) \otimes E\left(\lambda_{1}, \lambda_{2}\right) \otimes P(\mu)
\end{aligned}
$$

as a graded algebra. Here $E\left(Q_{1}\right)_{*}=E\left(\xi_{2}\right)$ and $\operatorname{Ext}_{E\left(\xi_{2}\right)}^{* *}\left(\mathbb{F}_{2}, \mathbb{F}_{2}\right)=P\left(v_{1}\right)$ with $v_{1}=\left[\xi_{2}\right]$ in the cobar complex.

(b) The $E_{2}$-term of the Adams spectral sequence for $X=T H H(k o) \wedge Y$ is

$$
\begin{aligned}
E_{2}^{* *} & =\operatorname{Ext}_{A_{*}}^{* *}\left(\mathbb{F}_{2},\left(A / / E\left(Q_{1}\right)\right)_{*} \otimes E\left(\lambda_{1}, \lambda_{2}\right) \otimes P(\mu)\right) \\
& \cong \operatorname{Ext}_{E\left(Q_{1}\right)_{*}}^{* *}\left(\mathbb{F}_{2}, E\left(\lambda_{1}, \lambda_{2}\right) \otimes P(\mu)\right) \\
& \cong \operatorname{Ext}_{E\left(Q_{1}\right)_{*}}^{* *}\left(\mathbb{F}_{2}, \mathbb{F}_{2}\left\{1, \lambda_{1}, \lambda_{2}, \mu, \lambda_{1} \lambda_{2}, \lambda_{1} \mu, \lambda_{2} \mu, \lambda_{1} \lambda_{2} \mu\right\} \otimes P\left(\mu^{2}\right)\right) .
\end{aligned}
$$

Here

$$
\begin{aligned}
& \mathbb{F}_{2}\left\{1, \lambda_{1}, \lambda_{2}, \mu, \lambda_{1} \lambda_{2}, \lambda_{1} \mu, \lambda_{2} \mu, \lambda_{1} \lambda_{2} \mu\right\} \cong \\
& \mathbb{F}_{2}\left\{1, \lambda_{2}, \lambda_{1} \mu, \lambda_{1} \lambda_{2} \mu\right\} \oplus E\left(Q_{1}\right)_{*}\left\{\lambda_{1}, \lambda_{1} \lambda_{2}\right\}
\end{aligned}
$$


as $E\left(Q_{1}\right)_{*}$-comodules. For $\lambda_{1}, \lambda_{2}$ and $\mu^{2}$ are $A_{*}$-comodule primitives, while $\nu(\mu)$ maps to $1 \otimes \mu+\xi_{2} \otimes \lambda_{1}$ in $E\left(Q_{1}\right)_{*} \otimes H_{*}\left(T H H(k o) \wedge Y ; \mathbb{F}_{2}\right)$. So

$$
E_{2}^{* *}=\left(P\left(v_{1}\right) \otimes E\left(\lambda_{2}, \lambda_{1} \mu\right) \oplus E\left(\lambda_{2}\right)\left\{\lambda_{1}\right\}\right) \otimes P\left(\mu^{2}\right) \text {. }
$$

We let $\lambda_{3}=\lambda_{1} \mu$ to obtain the claimed formula.

To determine the differentials in the Adams spectral sequence for $\pi_{*}(T H H(k u) \wedge$ $M)$ or $\pi_{*}(T H H(k o) \wedge Y)$ we first compute the $v_{1}$-periodic homotopy. This is in turn easy to derive from the $K(1)$-homology. Recall that $B P_{*} B P \cong B P_{*}\left[t_{k} \mid\right.$ $k \geq 1]$ with $\left|t_{k}\right|=2\left(2^{k}-1\right)$ and $K(1)_{*}=\mathbb{F}_{2}\left[v_{1}, v_{1}^{-1}\right]$ with $\left|v_{1}\right|=2$.

Lemma 8.9 For $p=2$ there are isomorphisms of $K(1)_{*}$-algebras

$$
K(1)_{*}(k u) \cong K(1)_{*}\left[t_{k} \mid k \geq 1\right] /\left(v_{1} t_{k}^{2}=v_{1}^{2^{k}} t_{k}\right) \cong K(1)_{*}\left[u_{k} \mid k \geq 1\right] /\left(u_{k}^{2}=u_{k}\right)
$$

and

$$
K(1)_{*}(k o) \cong K(1)_{*}\left[t_{k} \mid k \geq 2\right] /\left(v_{1} t_{k}^{2}=v_{1}^{2^{k}} t_{k}\right) \cong K(1)_{*}\left[u_{k} \mid k \geq 2\right] /\left(u_{k}^{2}=u_{k}\right) .
$$

Proof We first follow the proof of [MS93 5.3]. We have $K(1)_{*}(B P) \cong$ $K(1)_{*} \otimes_{B P_{*}} B P_{*}(B P) \cong K(1)_{*}\left[t_{k} \mid k \geq 1\right]$, where $\left|t_{k}\right|=2\left(2^{k}-1\right)$. The spectrum $k u_{(2)}=B P\langle 1\rangle$ can be constructed from $B P$ by Baas-Sullivan cofiber sequences killing the classes $v_{k+1}$ for $k \geq 1$, which map to

$$
\eta_{R}\left(v_{k+1}\right) \equiv v_{1} t_{k}^{2}+v_{1}^{2^{k}} t_{k} \bmod \left(\eta_{R}\left(v_{2}\right), \ldots, \eta_{R}\left(v_{k}\right)\right)
$$

by $\mathrm{Ra04}, 6.1 .13]$. So each $\eta_{R}\left(v_{k+1}\right) \in K(1)_{*}(B P)$ is not a zero divisor mod $\left(\eta_{R}\left(v_{2}\right), \ldots, \eta_{R}\left(v_{k}\right)\right)$, and

$$
K(1)_{*}(k u) \cong K(1)_{*}\left[t_{k} \mid k \geq 1\right] /\left(v_{1} t_{k}^{2}=v_{1}^{2^{k}} t_{k}\right) .
$$

Substituting $u_{k}=v_{1}^{1-2^{k}} t_{k}$, the relations become $u_{k}^{2}=u_{k}$ for each $k \geq 1$.

The cofiber sequence

$$
\Sigma k o \stackrel{\eta}{\longrightarrow} k o \stackrel{c}{\longrightarrow} k u \stackrel{\partial}{\longrightarrow} \Sigma^{2} k o
$$

induces a short exact sequence

$$
0 \rightarrow K(1)_{*}(k o) \stackrel{c_{*}}{\longrightarrow} K(1)_{*}(k u) \stackrel{\partial_{*}}{\longrightarrow} \Sigma^{2} K(1)_{*}(k o) \rightarrow 0
$$

since multiplication by $\eta$ is zero in $K(1)$-homology. The connecting map $\partial$ right multiplies by $S q^{2}$ in mod 2 cohomology, so right "comultiplies" with the dual class $\bar{\xi}_{1}^{2}$ in mod 2 homology, which corresponds to $t_{1}$ in $B P_{*} B P$ [Za72, p. 488]. From the coproduct formula for $\Delta\left(t_{j}\right)$ [Ra04, A2.1.27(e)] it follows that $\partial_{*}\left(t_{1}\right)=\Sigma^{2}(1)$ while $\partial_{*}\left(t_{k}\right)=0$ for $k \geq 2$. Hence we can identify $K(1)_{*}(k o)$ with the claimed subalgebra of $K(1)_{*}(k u)$, via the $K(1)_{*}$-algebra homomorphism $c_{*}$. 
Lemma 8.10 The unit maps $R \rightarrow T H H(R)$ induce isomorphisms

$$
K(1)_{*}(k u) \stackrel{\cong}{\longrightarrow} K(1)_{*} T H H(k u)
$$

and

$$
K(1)_{*}(k o) \stackrel{\cong}{\longrightarrow} K(1)_{*} T H H(k o) .
$$

Proof The proof of [MS93, 5.3] continues as follows. There is a $K(n)$-based Bökstedt spectral sequence

$$
E_{* *}^{2}=H H_{*}^{K(n)_{*}}\left(K(n)_{*}(R)\right) \Longrightarrow K(n)_{*} T H H(R)
$$

for every $S$-algebra $R$, derived like the one in (4.1), but by applying $K(n)$ homology to the skeleton filtration of $\operatorname{THH}(R)$. The identification of the $E^{2}$ term uses the Künneth formula for Morava $K$-theory. When $K(n)_{*}(R)$ is concentrated in degrees $* \equiv 0 \bmod \left|v_{n}\right|$, then $K(n)_{*}(R) \cong K(n)_{*} \otimes_{\mathbb{F}_{p}} K(n)_{0}(R)$ and we can rewrite the $E^{2}$-term as

$$
E_{* *}^{2} \cong K(n)_{*} \otimes_{\mathbb{F}_{p}} H H_{*}^{\mathbb{F}_{p}}\left(K(n)_{0}(R)\right) .
$$

This is the case for $n=1, p=2$ and $R=k u$, when $K(1)_{0}(k u)$ is the colimit over $m$ of the algebras $\mathbb{F}_{2}\left[u_{k} \mid 1 \leq k \leq m\right] /\left(u_{k}^{2}=u_{k}\right) \cong \prod_{i=1}^{2^{m}} \mathbb{F}_{2}$. Then for each $m$ the unit map $\prod_{i=1}^{2^{m}} \mathbb{F}_{2} \rightarrow H H_{*}^{\mathbb{F}_{2}}\left(\prod_{i=1}^{2^{m}} \mathbb{F}_{2}\right)$ is an isomorphism, so by passage to the colimit the unit map

$$
K(1)_{0}(k u) \rightarrow H H_{*}^{\mathbb{F}_{2}}\left(K(1)_{0}(k u)\right)
$$

is an isomorphism. Hence the $K(1)$-based Bökstedt spectral sequence collapses at the edge $s=0$, and the unit map $k u \rightarrow T H H(k u)$ induces the asserted isomorphism.

Likewise, $K(1)_{0}(k o)$ is the colimit of the algebras $\mathbb{F}_{2}\left[u_{k} \mid 2 \leq k \leq m\right] /\left(u_{k}^{2}=\right.$ $\left.u_{k}\right) \cong \prod_{i=1}^{2^{m-1}} \mathbb{F}_{2}$, and the same argument shows that the unit map $k o \rightarrow$ $T H H(k o)$ induces an isomorphism in $K(1)$-homology.

The mod 2 Moore spectrum $M$ admits a degree 8 self-map $v_{1}^{4}: M \rightarrow \Sigma^{-8} M$ that induces multiplication by $v_{1}^{4}$ in $k u_{*}(M)=k(1)_{*}=P\left(v_{1}\right)$. Smashing with $C_{\eta}$ yields a self-map $v_{1}^{4}: Y \rightarrow \Sigma^{-8} Y$, which admits a fourth root $v_{1}: Y \rightarrow \Sigma^{-2} Y$ (up to nilpotent maps). It induces multiplication by $v_{1}$ in $k o_{*}(Y)=k(1)_{*}=$ $P\left(v_{1}\right)$. See [DM82, 1.2].

Lemma 8.11 Let $X$ be a spectrum such that $K(1)_{*}(X)=0$. Then $v_{1}^{-1} \pi_{*}(X \wedge$ $Y)=0$ and $v_{1}^{-4} \pi_{*}(X \wedge M)=0$. 
Proof Recall that $j$ is the homotopy fiber of the map $\psi^{3}-1: k o_{(2)} \rightarrow \operatorname{bspin}_{(2)}$. The unit map $e: S \rightarrow j$ induces an equivalence of mapping telescopes

$$
v_{1}^{-1}(S \wedge Y) \stackrel{\simeq}{\longrightarrow} v_{1}^{-1}(j \wedge Y) .
$$

See e.g. the case $n=0$ of Mah82, 1.4]. Here $v_{1}^{-1}\left(k o_{(2)} \wedge Y\right) \simeq v_{1}^{-1} k(1)=K(1)$ and likewise $v_{1}^{-1}\left(\operatorname{bspin}_{(2)} \wedge Y\right) \simeq K(1)$, so there is a cofiber sequence of spectra

$$
v_{1}^{-1} Y \rightarrow K(1) \stackrel{\psi}{\longrightarrow} K(1) \text {. }
$$

Furthermore, $v_{1}^{-1} Y \simeq v_{1}^{-4} Y$ sits in a cofiber sequence

$$
v_{1}^{-4} \Sigma M \stackrel{\eta}{\longrightarrow} v_{1}^{-4} M \rightarrow v_{1}^{-4} Y
$$

where $\eta$ is nilpotent $\left(\eta^{4}=0\right)$.

From the first cofiber sequence it follows that if $K(1)_{*}(X)=0$, then $\pi_{*}(X \wedge$ $\left.v_{1}^{-1} Y\right)=v_{1}^{-1} \pi_{*}(X \wedge Y)=0$. From the second cofiber sequence it then follows that multiplication by $\eta$ is an isomorphism on $\pi_{*}\left(X \wedge v_{1}^{-4} M\right)=v_{1}^{-4} \pi_{*}(X \wedge M)$. Since $\eta$ is nilpotent this implies that $v_{1}^{-4} \pi_{*}(X \wedge M)=0$.

Corollary 8.12 The unit maps induce isomorphisms

$$
K(1)_{*}=v_{1}^{-4} \pi_{*}(k u \wedge M) \stackrel{\cong}{\longrightarrow} v_{1}^{-4} \pi_{*}(T H H(k u) \wedge M)
$$

and

$$
K(1)_{*}=v_{1}^{-1} \pi_{*}(k o \wedge Y) \stackrel{\cong}{\longrightarrow} v_{1}^{-1} \pi_{*}(T H H(k o) \wedge Y) .
$$

Proof Apply Lemmas 8.10 and 8.11 to the cofiber of the unit map $R \rightarrow$ $T H H(R)$, for $R=k u$ and $R=k o$, respectively.

Theorem 8.13 Consider the Adams spectral sequence

$$
E_{2}^{* *}=P\left(v_{1}\right) \otimes E\left(\lambda_{1}, \lambda_{2}\right) \otimes P(\mu)
$$

for $\pi_{*}(T H H(k u) \wedge M)$, with $\lambda_{1}=\sigma \bar{\xi}_{1}^{2}, \lambda_{2}=\sigma \bar{\xi}_{2}^{2}, \mu=\sigma \bar{\xi}_{3}+\bar{\xi}_{1} \cdot \sigma \bar{\xi}_{2}^{2}$ and $v_{1}=\left[\xi_{2}\right]$ in bidegrees $(s, t)=(0,3),(0,7),(0,8)$ and $(1,3)$, respectively. Recursively define

$$
\lambda_{n}=\lambda_{n-2} \mu^{2^{n-3}}
$$

for $n \geq 3$. Likewise define $r(1)=2, r(2)=4, r(n)=2^{n}+r(n-2), s(1)=3$, $s(2)=7$ and $s(n)=2^{n}+s(n-2)$, for $n \geq 3$. So $\lambda_{n}$ has bidegree $(0, s(n))$ and $2 r(n)+s(n)=2^{n+2}-1$. 
Then the Adams spectral sequence has differentials generated by

$$
d^{r(n)}\left(\mu^{2^{n-1}}\right)=v_{1}^{r(n)} \lambda_{n}
$$

for all $n \geq 1$. This leaves the $E_{\infty}$-term

$$
E_{\infty}^{* *}=P\left(v_{1}\right)\{1\} \oplus \bigoplus_{n=1}^{\infty} P_{r(n)}\left(v_{1}\right)\left\{\lambda_{n}\right\} \otimes E\left(\lambda_{n+1}\right) \otimes P\left(\mu^{2^{n}}\right) .
$$

Hence $\pi_{*}(T H H(k u) \wedge M)=\pi_{*}(T H H(k u) ; \mathbb{Z} / 2)$ is generated as a $P\left(v_{1}\right)$-module by elements $1, x_{n, m}=\lambda_{n} \mu^{2^{n} m}$ and $x_{n, m}^{\prime}=\lambda_{n} \lambda_{n+1} \mu^{2^{n} m}$ for $n \geq 1$ and $m \geq 0$. Here $\left|x_{n, m}\right|=s(n)+2^{n+3} m$ and $\left|x_{n, m}^{\prime}\right|=s(n)+s(n+1)+2^{n+3} m$. The module structure is generated by the relations $v_{1}^{r(n)} x_{n, m}=0$ and $v_{1}^{r(n)} x_{n, m}^{\prime}=0$ for $n \geq 1, m \geq 0$.

Proof The classes $\lambda_{1}, \lambda_{2}$ and $\mu$ were introduced in Proposition 8.7, and the Adams spectral sequence $E_{2}$-term was found in Lemma 8.8. By Corollary 8.12 the abutment $\pi_{*}(T H H(k u) \wedge M)$ is all $v_{1}$-torsion, except the direct summand $\pi_{*}(k u \wedge M)=P\left(v_{1}\right)$, which is included by the unit map. Hence every class $\lambda_{n}$ is $v_{1}$-torsion, so there is some integer $r(n)$ such that $v_{1}^{r(n)} \lambda_{n}$ is hit by a differential.

Suppose by induction that the $d^{r(k)}$-differentials for $1 \leq k<n$ have been found, leaving the term

$$
\begin{aligned}
E_{r(n-1)+1}^{* *}= & P\left(v_{1}\right) \otimes E\left(\lambda_{n}, \lambda_{n+1}\right) \otimes P\left(\mu^{2^{n-1}}\right) \\
& \oplus \bigoplus_{k=1}^{n-1} P_{r(k)}\left(v_{1}\right)\left\{\lambda_{k}\right\} \otimes E\left(\lambda_{k+1}\right) \otimes P\left(\mu^{2^{k}}\right) .
\end{aligned}
$$

Consider the $P\left(v_{1}\right)$-module generated by $\lambda_{n}$. Let $r$ be minimal such that $v_{1}^{r} \lambda_{n}$ is a boundary. The source $x$ of such a differential cannot be divisible by $v_{1}$, since $r$ is minimal, so $d^{r}(x)=v_{1}^{r} \lambda_{n}$ where $x$ has Adams filtration $s=0$ and even total degree. Furthermore, $v_{1}^{r} \lambda_{n}$ is not $v_{1}$-torsion at this term, so $x$ cannot be $v_{1}$-torsion. Likewise, $\lambda_{n+1}$ does not annihilate $v_{1}^{r} \lambda_{n}$, so $\lambda_{n+1}$ cannot annihilate $x$ either. This forces $x \in P\left(\mu^{2^{n-1}}\right)$. By Lemma 8.5. $T H H(k u) \wedge M$ is a $\mu$-spectrum, so $d^{r}$ is a derivation and the Leibniz rule shows that $x=\mu^{2^{n-1}}$, since $d^{r}$ on any higher power of $\mu^{2^{n-1}}$ must be divisible by $\mu^{2^{n-1}}$. Hence $d^{r}\left(\mu^{2^{n-1}}\right)=v_{1}^{r} \lambda_{n}$, and by a degree count we must have $r=r(n)$.

To complete the induction step, we must compute the $E_{r(n)+1}$-term of the Adams spectral sequence. The $d^{r(n)}$-differential does not affect the summands $P_{r(k)}\left(v_{1}\right)\left\{\lambda_{k}\right\} \otimes E\left(\lambda_{k+1}\right) \otimes P\left(\mu^{2^{k}}\right)$ for $1 \leq k<n$, and is zero on $E\left(\lambda_{n+1}\right) \otimes$ 
$P\left(\mu^{2^{n}}\right)$. It acts on $P\left(v_{1}\right)\left\{1, \lambda_{n}, \mu^{2^{n-1}}, \lambda_{n} \mu^{2^{n-1}}\right\}$, leaving $P_{r(n)}\left(v_{1}\right)\left\{\lambda_{n}\right\} \oplus P\left(v_{1}\right) \otimes$ $E\left(\lambda_{n+2}\right)$, where by definition $\lambda_{n+2}=\lambda_{n} \mu^{2^{n-1}}$. This shows that the term $P\left(v_{1}\right) \otimes E\left(\lambda_{n}, \lambda_{n+1}\right) \otimes P\left(\mu^{2^{n-1}}\right)$ at the $E_{r(n-1)+1}$-term gets replaced by the direct sum of $P\left(v_{1}\right) \otimes E\left(\lambda_{n+1}, \lambda_{n+2}\right) \otimes P\left(\mu^{2^{n}}\right)$ and $P_{r(n)}\left(v_{1}\right)\left\{\lambda_{n}\right\} \otimes E\left(\lambda_{n+1}\right) \otimes$ $P\left(\mu^{2^{n}}\right)$.

Theorem 8.14 Consider the Adams spectral sequence

$$
E_{2}^{* *}=\left(P\left(v_{1}\right) \otimes E\left(\lambda_{2}, \lambda_{3}\right) \oplus E\left(\lambda_{2}\right)\left\{\lambda_{1}\right\}\right) \otimes P\left(\mu^{2}\right)
$$

for $\pi_{*}(T H H(k o) \wedge Y)$, with $\lambda_{1}=\sigma \bar{\xi}_{1}^{4}, \lambda_{2}=\sigma \bar{\xi}_{2}^{2}+\bar{\xi}_{1}^{2} \cdot \sigma \bar{\xi}_{1}^{4}, \lambda_{3}=\sigma \bar{\xi}_{1}^{4}\left(\sigma \bar{\xi}_{3}+\bar{\xi}_{1} \cdot \sigma \bar{\xi}_{2}^{2}\right)$, $\mu^{2}=\left(\sigma \bar{\xi}_{3}\right)^{2}$ and $v_{1}=\left[\xi_{2}\right]$ in bidegrees $(s, t)=(0,5),(0,7),(0,13),(0,16)$ and $(1,3)$, respectively. Recursively define

$$
\lambda_{n}=\lambda_{n-2} \mu^{2^{n-3}}
$$

for $n \geq 4$. Likewise define $r(1)=1, r(2)=4, r(n)=2^{n}+r(n-2), s(1)=5$, $s(2)=7$ and $s(n)=2^{n}+s(n-2)$, for $n \geq 3$. So $\lambda_{n}$ has bidegree $(0, s(n))$ and $2 r(n)+s(n)=2^{n+2}-1$.

Then the Adams spectral sequence has differentials generated by

$$
d^{r(n)}\left(\mu^{2^{n-1}}\right)=v_{1}^{r(n)} \lambda_{n}
$$

for all $n \geq 2$. This leaves the $E_{\infty}$-term

$$
E_{\infty}^{* *}=P\left(v_{1}\right)\{1\} \oplus \bigoplus_{n=1}^{\infty} P_{r(n)}\left(v_{1}\right)\left\{\lambda_{n}\right\} \otimes E\left(\lambda_{n+1}\right) \otimes P\left(\mu^{2^{n}}\right) .
$$

Hence $\pi_{*}(T H H(k o) \wedge Y)=\pi_{*}(T H H(k o) ; Y)$ is generated as a $P\left(v_{1}\right)$-module by elements $1, x_{n, m}=\lambda_{n} \mu^{2^{n} m}$ and $x_{n, m}^{\prime}=\lambda_{n} \lambda_{n+1} \mu^{2^{n} m}$ for $n \geq 1$ and $m \geq 0$. Here $\left|x_{n, m}\right|=s(n)+2^{n+3} m$ and $\left|x_{n, m}^{\prime}\right|=s(n)+s(n+1)+2^{n+3} m$. The module structure is generated by the relations $v_{1}^{r(n)} x_{n, m}=0$ and $v_{1}^{r(n)} x_{n, m}^{\prime}=0$ for $n \geq 1, m \geq 0$.

Proof Starting with an imagined $E_{1}$-term

$$
E_{1}^{* *}=P\left(v_{1}\right) \otimes E\left(\lambda_{1}, \lambda_{2}\right) \otimes P(\mu)
$$

and differential $d_{1}(\mu)=v_{1} \lambda_{1}$, the proof is the same as for Theorem 8.13 . 


\section{References}

[AP76] J F Adams, S B Priddy, Uniqueness of BSO, Math. Proc. Cambridge Philos. Soc. 80 (1976) 475-509 MathReview

[An02] V Angeltveit, Hopf algebra structure on topological Hochschild homology, Cand. scient. thesis, University of Oslo (2002)

[AT65] S Araki, H Toda, Multiplicative structures in $\bmod q$ cohomology theories. I, Osaka J. Math. 2 (1965) 71-115 MathReview

[Au] Ch Ausoni, Topological Hochschild homology of connective complex K-theory, Amer. J. Math. (to appear)

[AR02] C Ausoni, J Rognes, Algebraic $K$-theory of topological $K$-theory, Acta Math. 188 (2002) 1-39 MathReview

[BM72] N A Baas, I Madsen, On the realization of certain modules over the Steenrod algebra, Math. Scand. 31 (1972) 220-224 MathReview

[BJ02] A Baker, A Jeanneret, Brave new Hopf algebroids and extensions of $M U$ algebras, Homology Homotopy Appl. 4 (2002) 163-173 MathReview

[BaRi] A Baker, B Richter, $\Gamma$-cohomology of rings of numerical polynomials and $E_{\infty}$ structures on $K$-theory, Comment. Math. Helv. (to appear)

[Bo1] M Bökstedt, Topological Hochschild homology, University of Bielefeld preprint (ca. 1986)

[Bo2] M Bökstedt, The topological Hochschild homology of $\mathbb{Z}$ and $\mathbb{Z} / p$, University of Bielefeld preprint (ca. 1986)

[BHM93] M Bökstedt, W C Hsiang, I Madsen, The cyclotomic trace and algebraic $K$-theory of spaces, Invent. Math. 111 (1993) 465-539 MathReview

[BM94] M Bökstedt, I Madsen, Topological cyclic homology of the integers, Astérisque (1994) 7-8, 57-143 MathReview

[BMMS86] R R Bruner, J P May, J E McClure, M Steinberger, $H_{\infty}$ ring spectra and their applications, Lecture Notes in Mathematics 1176, Springer-Verlag, Berlin (1986) MathReview

[BR05] R R Bruner, J Rognes, Differentials in the homological homotopy fixed point spectral sequence, Algebr. Geom. Topol. 5 (2005) 653-690 MathReview

[CE56] H Cartan, S Eilenberg, Homological algebra, Princeton University Press, Princeton, N. J. (1956) MathReview

[Da75] D M Davis, The cohomology of the spectrum bJ, Bol. Soc. Mat. Mexicana (2) 20 (1975) 6-11 MathReview

[Du97] B I Dundas, Relative K-theory and topological cyclic homology, Acta Math. 179 (1997) 223-242 MathReview

[DM82] D M Davis, M Mahowald, $v_{1}$ - and $v_{2}$-periodicity in stable homotopy theory, Amer. J. Math. 103 (1981) 615-659 MathReview 
[EKMM97] A D Elmendorf, I Kriz, M A Mandell, J P May, Rings, modules, and algebras in stable homotopy theory, Mathematical Surveys and Monographs 47, American Mathematical Society, Providence, RI (1997) MathReview

[FV] Z Fiedorowicz, R M Vogt, Topological Hochschild homology of $E_{n}$ ring spectra, arXiv:math.AT/0410367

[HM97] L Hesselholt, I Madsen, On the $K$-theory of finite algebras over Witt vectors of perfect fields, Topology 36 (1997) 29-101 MathReview

[HM03] L Hesselholt, I Madsen, On the K-theory of local fields, Ann. of Math. (2) 158 (2003) 1-113 MathReview

[HSS00] M Hovey, B Shipley, J Smith, Symmetric spectra, J. Amer. Math. Soc. 13 (2000) 149-208 MathReview

[Hu96] T J Hunter, On the homology spectral sequence for topological Hochschild homology, Trans. Amer. Math. Soc. 348 (1996) 3941-3953 MathReview

[JW73] D C Johnson, W S Wilson, Projective dimension and Brown-Peterson homology, Topology 12 (1973) 327-353 MathReview

[La03] A Lazarev, Towers of MU-algebras and the generalized Hopkins-Miller theorem, Proc. London Math. Soc. (3) 87 (2003) 498-522 MathReview

[LMS86] L G Lewis, Jr, J P May, M Steinberger, J E McClure, Equivariant stable homotopy theory, Lecture Notes in Mathematics 1213, Springer-Verlag, Berlin (1986) MathReview

[L-N05] S Lunøe-Nielsen, A homological approach to topological cyclic theory, Dr. scient. thesis, University of Oslo (2005)

[Mac75] S Mac Lane, Homology, Classics in Mathematics, Springer-Verlag, Berlin (1995) MathReview

[MaMi76] M Mahowald, R J Milgram, Operations which detect Sq4 in connective $K$-theory and their applications, Quart. J. Math. Oxford Ser. (2) 27 (1976) 415-432 MathReview

[Mah82] M Mahowald, The image of $J$ in the EHP sequence, Ann. of Math. (2) 116 (1982) 65-112 MathReview

[May77] J P May, $E_{\infty}$ ring spaces and $E_{\infty}$ ring spectra, Springer-Verlag, Berlin (1977) MathReview

[Mc97] R McCarthy, Relative algebraic K-theory and topological cyclic homology, Acta Math. 179 (1997) 197-222 MathReview

[MSV97] J McClure, R Schwänzl, R Vogt, $T H H(R) \cong R \otimes S^{1}$ for $E_{\infty}$ ring spectra, J. Pure Appl. Algebra 121 (1997) 137-159 MathReview

[MS93] J E McClure, R E Staffeldt, On the topological Hochschild homology of bu. I, Amer. J. Math. 115 (1993) 1-45 MathReview

[Mi60] J Milnor, The Steenrod algebra and its dual, Ann. of Math. (2) 67 (1958) 150-171 MathReview 
[MiMo65] J W Milnor, J C Moore, On the structure of Hopf algebras, Ann. of Math. (2) 81 (1965) 211-264 MathReview

[Ok79] S Oka, Ring spectra with few cells, Japan. J. Math. (N.S.) 5 (1979) 81-100 MathReview

[Ra04] D C Ravenel, Complex cobordism and stable homotopy groups of spheres, Pure and Applied Mathematics 121, Academic Press, Orlando, FL (1986) MathReview

[Re01] C Rezk, Supplementary notes for Math 512, http://www.math.uiuc.edu/ rezk/512-spr2001-notes.pdf

[Rob89] A Robinson, Obstruction theory and the strict associativity of Morava Ktheories, from: "Advances in homotopy theory (Cortona, 1988)", London Math. Soc. Lecture Note Ser. 139, Cambridge Univ. Press, Cambridge (1989) 143-152 MathReview

[Rog03] J Rognes, The smooth Whitehead spectrum of a point at odd regular primes, Geom. Topol. 7 (2003) 155-184 MathReview

[Sta63] J D Stasheff, Homotopy associativity of $H$-spaces. I, II, Trans. Amer. Math. Soc. 108 (1963), 275-292; ibid. 108 (1963) 293-312 MathReview

[Sta97] J Stasheff, From operads to "physically" inspired theories, from: "Operads: Proceedings of Renaissance Conferences (Hartford, CT/Luminy, 1995)", Contemp. Math. 202, Amer. Math. Soc., Providence, RI (1997) 53-81 MathReview

[Sto63] RE Stong, Determination of $H^{*}\left(\mathrm{BO}(k, \cdots, \infty), Z_{2}\right)$ and $H^{*}\left(\mathrm{BU}(k, \cdots, \infty), Z_{2}\right)$, Trans. Amer. Math. Soc. 107 (1963) 526-544 MathReview

[Str99] N P Strickland, Products on MU-modules, Trans. Amer. Math. Soc. 351 (1999) 2569-2606 MathReview

[Wi75] W S Wilson, The $\Omega$-spectrum for Brown-Peterson cohomology. II, Amer. J. Math. 97 (1975) 101-123 MathReview

[Za72] R Zahler, The Adams-Novikov spectral sequence for the spheres, Ann. of Math. (2) 96 (1972) 480-504 MathReview

Department of Mathematics, Massachusetts Institute of Technology

Cambridge, MA 02139-4307, USA

and

Department of Mathematics, University of Oslo

Blindern NO-0316, Norway

Email: vigleik@math.mit.edu and rognes@math.uio.no

Received: 16 July 2004 Revised: 21 September 2005 